\title{
X-Ray and Neutron Diffuse Scattering Measurements
}

\begin{tabular}{|r|l|}
\hline Journal: & Characterization of Materials \\
\hline Manuscript ID: & Draft \\
\hline Wiley - Manuscript type: & Review Article \\
\hline $\begin{array}{r}\text { Date Submitted by the } \\
\text { Author: }\end{array}$ & $\mathrm{n} / \mathrm{a}$ \\
\hline Complete List of Authors: & $\begin{array}{l}\text { Ice, Gene; Oak Ridge National Laboratory, Material Science and } \\
\text { Technology Division }\end{array}$ \\
\hline Keywords: & \\
\hline
\end{tabular}

SCHOLARONE
Manuscripts 


\section{X-RAY AND NEUTRON DIFFUSE SCATTERING MEASUREMENTS}

\section{INTRODUCTION}

Diffuse scattering from crystalline solid solutions is used to measure local compositional order among the atoms, dynamic displacements (phonons), and mean species-dependent static displacements. It can also reveal defect densities and the presence of second phases, vacancies and insterstitials. In locally ordered alloys, fluctuations of composition and interatomic distances break the long-range symmetry of the crystal within local regions and contribute to the total energy of the alloy (Zunger, 1994). Local ordering can be a precursor to a lower temperature equilibrium structure that may be unattainable because of slow atomic diffusion. Discussions of the usefulness of local chemical and displacive correlations within alloy theory are given in Chapter 2 (see PREDICTION OF PHASE DIAGRAMS and COMPUTATION OF DIFFUSE INTENSITIES IN ALLOYS). In addition to local atomic correlations, neutron diffuse scattering methods can be used to study the local short-range correlations of the magnetic moments. Interstitial defects and vacancies as opposed to the substitutional disorder defects described above, also disrupt the long-range periodicity of a crystalline material and give rise to diffusely scattered x rays, neutrons (Larson 2009), and electrons (electron scattering is not covered in this unit; Schweika, 1998).

Use of tunable synchrotron radiation to change the $\mathrm{x}$-ray scattering contrast between elements has greatly improved the measurement of bond distances between the three types of atom pairs found in crystalline binary alloys (Ice et al., 1992). The estimated standard deviation of the first-order (first moment) mean static displacements from this technique approaches $\pm 0.001 \AA(0.0001 \mathrm{~nm})$, which is an order of magnitude more precise than results obtained with extended $x$-ray absorption fine structure (EXAFS; XAFS SPECTROSCOPY) measurements. In addition, both the radial and tangential displacements can be reliably determined to five or more near-neighbor shells (Jiang et al., 1996). In a binary A-B alloy, the number of A or B nearneighbor atoms to, for example, an A atom can be determined to even less than 1 atom in 100. The second moment of the static displacements, which gives rise to Huang scattering, is also measurable (Schweika, 1998). Measurements of diffuse scattering can also reveal the tensorial displacements associated with substitutional and interstitial defects. This information can be used to model the statistical arrangements of the atoms on a local scale.

An example of chemical local ordering is given in Figure 1, where the probability $P_{l m n}^{\mathrm{AB}}$ of finding a B atom out to the sixth Imn shell around an A atom goes from a preference for A atoms (clustering) to a preference for $\mathrm{B}$ atoms (short-range order) for a body-centered cubic (bcc) $\mathrm{A}_{50} \mathrm{~B}_{50}$ alloy. The real-space representation of the atom positions is derived from a Monte Carlo simulation of the $P_{l m n}^{\mathrm{AB}}$ values (Gehlen and Cohen, 1965). The intensity distribution in reciprocal space is then calculated (Robertson et al., 1998). In the upper panel, the probability of finding a $\mathrm{B}$ atom as the first neighbor to an $\mathrm{A}$ atom is $40 \%(10 \%$ clustering; $P_{111}^{\mathrm{AB}}=0.4$ ). Atoms are located on a (110) plane so that first-neighbor pairs are shown. The middle panel depicts the random alloy where $P_{l m n}^{\mathrm{AB}}=-0.5$ for the first six shells $(l m n)$. The lower panel shows the case where $P_{l m n}^{\mathrm{AB}}=0.6$ (a preference for unlike atom pairs). The intensity distribution in the (100) plane of reciprocal space (with the fundamental Bragg maxima removed) is shown in the right column of Figure 1. Note that a preference for like nearest neighbors causes the scattering to be centered near the fundamental Bragg maxima, such as at the origin, 0,0. A preference for unlike first-neighbor pairs causes the diffuse scattering to peak at the superlattice reflections for an ordered structure. Models, such as those shown in Figure 1, are used to understand materials properties and their response to heat treatment, mechanical deformation, and magnetic fields. These local configurations are useful to test advances in theoretical models of crystalline alloys as discussed in COMPUTATION OF DIFFUSE INTENSITIES IN ALLOYS.

The diffraction pattern from a crystalline material with perfect periodicity, such as nearly perfect single-crystal $\mathrm{Si}$, consists of sharp Bragg maxima associated with long-range periodicity. With Bragg's law, we can determine the size of the average unit cell. Because of thermal motion, atom positions are smeared and Bragg maxima are reduced. In alloys with different atomic sizes, static displacements will also reduce Bragg intensity. The intensity, which is lost from the Bragg reflections, is diffusely distributed. Shown schematically in Figure 2A is a solid solution of two kinds of atoms displaced from the sites of the average lattice in such a way that the average plane of atoms is regularly spaced with a constant " $d$ " spacing existing over hundreds of planes. As shown schematically in Figure 2B, there is weak diffuse scattering but no broadening of the fundamental Bragg reflections, as would be the case for more extended defects such as stacking faults, high dislocation densities, displacive transformations, and incoherent precipitates, among others (Warren, 1969). In cases where the fundamental Bragg reflections are broadened, our uncertainty in the size of the average lattice increases and the precision of the measured pair separation is reduced.

This unit will concentrate on the use of diffuse x-ray and neutron scattering from single crystals to measure local chemical correlations and chemically specific static displacements. Particular emphasis will be placed on the use of resonant (anomalous) $x$-ray techniques to extract information on atomic size from binary solid solutions with short-range order. Here the alloys have a well-defined average lattice but have local fluctuations in composition and displacements from the average lattice. In stoichiometric crystals with long-range periodicity, sharp superlattice Bragg reflections appear. If the compositional order is correlated only over short distances, the superlattice reflections are so broadened that measurements throughout a symmetry related volume in reciprocal space are required to determine their distributions. In addition, the displacement of the atom pairs (e.g., the A-A, A-B, and B-B pairs in a binary alloy) from the sites of the average lattice because of different atom sizes also contributes to the distribution of this diffuse scattering. By separating this diffuse intensity into its component partsthat associated with the chemical preference for A-A, A-B, and $\mathrm{B}-\mathrm{B}$ pairs for the various near-neighbor shells and that associated with the static and dynamic displacements of the atoms from the sites of the average lattice-we are able to recover pair correlation probabilities for the three kinds of pairs in a binary alloy. The interpretation of diffuse scattering associated with dynamic displacements of atoms from their average crystal sites will be discussed only briefly in this unit.

\section{Competitive and Related Techniques}

Other techniques that measure local chemical order and bond distances exist. In EXAFS, outgoing photoejected electrons are scattered by the surrounding near neighbors (most from first and to a lesser extent from second nearest neighbors). This creates modulations of the $\mathrm{x}$-ray absorption cross-section, typically extending for $\sim 1000 \mathrm{eV}$ above the edge, and gives information about both local chemical order and bond distances (see XAFS SPECTROSCOPY for details). Usually, the phase and amplitudes for the interference of the photo-ejected electrons must be extracted from model systems, which necessitates measurements on intermetallic (ordered) compounds of known bond distances and neighboring atoms. The choice of an incident x-ray energy specific to an elemental absorption edge makes EXAFS information specific to that elemental constituent. For an alloy of $\mathrm{A}$ and $\mathrm{B}$ atoms, the EXAFS for an absorption edge of an A atom would be sensitive to the $\mathrm{A}$ and $\mathrm{B}$ atoms neighboring the $\mathrm{A}$ atoms. Separation of the signal into A-A and B-B pairs is typically done by using dilute alloys containing 2 at.\% or less of the constituent of interest (e.g., the A atoms). The EXAFS signal is then interpreted as arising from the predominately $B$ neighborhood of an A atom, and analyzed in terms of the number of B first and second neighbors and their bond distances from the A atoms. Claims for the accuracy of the EXAFS method vary between 0.01 and $0.02 \AA$ for bond distance and $\sim 10 \%$ for the first shell coordination number (number of atoms in the first shell; Scheuer and Lengeler, 1991). For crystalline solid-solution alloys, the crystal structure precisely determines the number of neighbors in each shell but not the kinds for nondilute alloys. For most alloys, the precision achieved with EXAFS is inadequate to determine the deviations of the interatomic spacings from the average lattice. Whenever EXAFS measurements are applicable and of sufficient precision to determine the information of interest, the ease and simplicity of this experiment compared 
with three-dimensional diffuse scattering measurements makes it an attractive tool. An EXAFS study of concentrated Au-Ni alloys revealed the kind of information available (Renaud et al., 1988).

Mössbauer spectroscopy is another method for obtaining near-neighbor information (MOSSBAUER SPECTROMETRY). Measurements of hyperfine field splitting caused by changes in the electric field gradient or magnetic field because of the different charge or magnetic states of the nuclear environments give information about the near-neighbor environments. Different chemical and magnetic environments of the nucleus produce different hyperfine structure, which is interpreted as a measure of the different chemical environments typical for first and second neighbors. The quantitative interpretation of Mössbauer spectra in terms of local order and bond distances is often ambiguous. The use of Mössbauer spectroscopy is limited to those few alloys where at least one of the constituents is a Mössbauer-active isotope. This spectroscopy is complimentary but does not compete with diffuse scattering measurements as a direct method for obtaining detailed information about nearneighbor chemical environments and bond distances (Drijver et al., 1977; Pierron-Bohnes et al., 1983).

Imaging techniques with electrons, such as $Z$ contrast microscopy and other high-resolution electron microscopy techniques (see Chapter 11), do not yet have the resolution for measuring the small displacements associated with crystalline solid solutions heterogeneity, but can be used to study the displacements associated with interfaces and surfaces. Imaging for high-resolution microscopy requires a thin sample about a dozen or more unit cells thick with identical atom occupations, and precludes obtaining information about short-range order and bond distances. Electron diffuse scattering measurements are difficult to record in absolute units and to separate from contributions to the diffuse background caused by straggling energy loss processes. Electron techniques provide extremely useful information on more extended defects as discussed in Chapter 11.

Field ion microscopy uses $\mathrm{He}$ or $\mathrm{Ne}$ gas atoms to image the small radius tip of the sample. Atom probes provide direct imaging of atom positions. Atoms are pulled from a small radius tip of the sample by an applied voltage and mass analyzed through a small opening. The position of the atom can be localized to $\sim 5 \AA$. Information on the species of an atom and its neighbors can be recovered. Reports of successful analysis of concentration waves and clusters in phase separating alloys have occurred where strongly enriched clusters of like atoms are as small as $5 \AA$ in diameter (Miller et al., 1996). However, information on small displacements cannot be obtained with atom probes. Scanning tunneling (see SCANNING TUNNELING MICROSCOPY) and atomic force microscopy can distinguish between the kinds of atoms on a surface and reveal their relative positions.

\section{PRINCIPLES OF THE METHOD}

In this section, we formulate the diffraction theory of diffuse scattering in a way that minimizes assumptions and maximizes the information obtained from a diffraction pattern without recourse to models. This approach can be extended with various theories and models for interpretation of the recovered information. Measurements of diffusely scattered radiation can reveal the kinds and number of defects. Since different defects give different signatures in diffuse scattering, separation of these signatures can simplify recovery of the phenomenological parameters describing the defect.

Availability of intense and tunable synchrotron x-ray sources, which allow the selection of $\mathrm{x}$-ray energies near absorption edges, permits the use of resonant scattering techniques to separate the contribution to the diffuse scattering from different kinds of pairs (e.g., the A-A, A-B, and B-B pairs of a binary alloy). Near an $\mathrm{x}$-ray $K$ absorption edge, the x-ray scattering factor of an atom can change by $\sim 8$ electron units (eu) and allows for scattering contrast control between atoms in an alloy. Adjustable contrast, either through resonant (anomalous) x-ray scattering or through isotopic substitution for neutrons, allows for precision measurement of chemically specific localinteratomic distances within the alloy. Figure 3 gives the realspace notation used in describing the atom positions and its reciprocal space notations used in describing the intensity distribution.

\section{Rays Versus Neutrons}

The choice of $\mathrm{x}$ rays or neutrons for a given experiment depends on instrumentation and source availability, the constituent elements of the sample, the information sought, the temperature of the experiment, the size of the sample, and isotopic availability and cost, among other considerations. Neutron scattering is particularly useful for measurements of low- $Z$ materials, for high-temperature measurements, and for measurements of magnetic ordering. X-ray scattering is preferred for measurements of small samples, for measurement of static displacements, and for good momentum transfer, $\mathbf{H}$, resolution.

\section{Chemical Order}

Recovery of the local chemical preference for atom neighbors has been predominately an $\mathrm{x}$-ray diffuse scattering measurement, although $\mathrm{x}$-ray and neutron measurements are complimentary. More than 60 systems have been studied with $\mathrm{x}$ rays and around 10 systems with neutrons. The choice between x-ray and neutron methods often depends upon which one gives the best contrast between the constituent elements and which one allows the greatest control over contrast-isotopic substitution for contrast change with neutrons (Cenedese et al., 1984) or resonant (synonymous with dispersion and anomalous) $\mathrm{x}$-ray techniques with $\mathrm{x}$-ray energies near to absorption edge energies (Ice et al., 1994). In general, x-ray scattering is favored by virtue of its better intensity and collimation, which allow for smaller samples and better momentum-transfer resolution. Neutron diffuse scattering has the advantage of discriminating against thermal diffuse scattering (TDS); there is a significant change in energy (wave-length) when neutrons are scattered by phonons (see PhONON STUDIES). For example, phonons in the few tens of millielectron volt energy range make an insignificant change in the energy of kiloelectron volt $\mathrm{x}$ rays but make a significant change in the $\sim 35-\mathrm{meV}$ energy of thermal neutrons, except near Bragg reflections. Thus, TDS of neutrons is easily rejected with crystal diffraction or time-of-flight techniques even at temperatures approaching and exceeding the Debye temperature. With $\mathrm{x}$ rays, however, TDS can be a major contribution and can obscure the Laue scattering. Magnetic short-range order can also be studied with neutrons in much the same way as the chemical short-range order. However, when the alloy is magnetic, extra effort is needed to separate the magnetic scattering from the nuclear scattering that gives the information on chemical pair correlations.

Neutrons can be used in combination with $\mathrm{x}$ rays to obtain additional scattering contrast. The $\mathrm{x}$-ray scattering factors increase with the atomic number of the elements (since it increases with the number of electrons), but neutron scattering cross-sections are not correlated with the atomic number as they are scattered from the nucleus. When an absorption edge of one of the elements is either too high for available x-ray energies or too low in energy for the reciprocal space of interest, or if enough different isotopes of the atomic species making up the alloy are not available or are too expensive, then a combination of both $\mathrm{x}$-ray and neutron diffuse scattering measurements may be a way to obtain the needed contrast. A more in-depth discussion of the properties of neutrons is given in Chapter 13. Information on chemical short-range order obtained with both $\mathrm{x}$ rays and neutrons and a general discussion of their merits is given by Kostorz (1996).

Local chemical order among the atoms including vacancies has been measured for $\sim 70$ metallic binary alloys and a few oxides. Only two ternary metallic systems have been measured in which the three independent pair probabilities between the three kinds of atoms have been recovered: an alloy of $\mathrm{Cr}_{21} \mathrm{Fe}_{56} \mathrm{Ni}_{23}$ with three different isotopic contents studied with neutron diffuse scattering measurements (Cenedese et al., 1984) and an alloy $\mathrm{Cu}_{47} \mathrm{Ni}_{29} \mathrm{Zn}_{34}$ studied with three $\mathrm{x}$-ray energies (Hashimoto et al, 1985).

\section{Bond Distances}

In binary alloys, recovery of the bond distances between A-A, A$\mathrm{B}$, and $\mathrm{B}-\mathrm{B}$ pairs requires measurement of the diffuse intensity at 
two different scattering contrasts to separate the A-A and B-B bond distances (as shown, later the A-B bond distance can be expressed as a linear combination of the other two distances to conserve volume). Such measurements require two matched samples of differing isotopic content to develop neutron contrast, whereas for $\mathrm{x}$ rays, the $\mathrm{x}$-ray energy need only be changed a fraction of a kilovolt near an absorption edge to produce a significant contrast change (Ice et al., 1994). Of course, one of the elemental constituents of the sample needs to have an absorption edge energy above $\sim 5 \mathrm{keV}$ to obtain sufficient momentum transfer for a full separation of the thermal contribution. However, more limited momentum-transfer data can be valuable for certain applications. Thus binary solid solutions where both elements have atomic numbers lower than that of $\operatorname{Cr}(Z=24)$ may require both an $x$-ray and a neutron measurement of the diffuse intensity to obtain data of sufficient contrast and momentum transfer. To date, there have been about 10 publications on the recovery of bond distances. Most have employed diffuse x-ray scattering measurements, but some employed neutrons and isotopically substituted samples (Müller et al., 1989).

\section{Diffuse X-ray (Neutron) Scattering Theory} for Crystalline Solid Solutions

In the kinematic approximation, the elastically scattered x-ray (neutron) intensity in electron units per atom from an ensemble of atoms is given by

$$
I(\mathbf{H})_{\text {Total }}=\left|\sum_{p} f_{p} e^{2 \pi \mathbf{H} \cdot \mathbf{r}_{p}}\right|^{2}=\sum_{p} \sum_{q} f_{p} f_{q}^{*} e^{2 \pi \mathbf{H} \cdot\left(\mathbf{r}_{p}-\mathbf{r}_{q}\right)}
$$

Here $f_{p}$ and $f_{q}^{*}$ denote the complex and complex-conjugate xray atomic scattering factors (or neutron scattering lengths), $p$ and $q$ designate the lattice sites from 0 to $N-1, \mathbf{r}_{p}$ and $\mathbf{r}_{q}$ are the atomic position vectors for those sites, and $\mathbf{H}$ is the momentum transfer or reciprocal lattice vector $|\mathbf{H}|=(2 \sin \theta) / \lambda$ (Fig. 3). For crystalline solid solutions with a well-defined long-range lattice (sharp Bragg reflections), the atom positions can be represented by $\mathbf{r}=\mathbf{R}+\delta$, where $\mathbf{R}$ is determined from the lattice constants and $\delta$ is both the thermal and static displacement of the atom from that average lattice. Equation 1 can be separated into terms of the average lattice $\mathbf{R}$ and the local fluctuations $\delta$,

$$
I(\mathbf{H})_{\text {Total }}=\sum_{p} \sum_{q} f_{p} f_{q}^{*} e^{2 \pi \mathbf{H} \boldsymbol{\bullet}\left(\delta_{p}-\delta_{q}\right)} e^{2 \pi \mathbf{H} \cdot\left(\mathbf{R}_{p}-\mathbf{R}_{q}\right)}
$$

We limit our discussion of the diffraction theory to crystalline binary alloys of $\mathrm{A}$ and $\mathrm{B}$ atoms with atomic concentration $C_{\mathrm{A}}$ and $C_{\mathrm{B}}$, respectively, and with complex x-ray atomic scattering factors of $f_{\mathrm{A}}$ and $f_{\mathrm{B}}$, (neutron scattering lengths $b_{\mathrm{A}}$ and $b_{\mathrm{B}}$ ). Since an x-ray or neutron beam of even a millimeter diameter intercepts $>10^{20}$ atoms, the double sum in Equation 2 involves $>10^{21}$ first-neighbor atom pairs (one at $p$, the other at $q$ ); the sum over all the atoms is a statistical description, which includes all possible atom pairs that can be formed (i.e., A-A, A-B, B-A, B$\mathrm{B}$; Warren, 1969). A preference for like or unlike neighboring pairs is introduced by the conditional probability term $P_{p q}^{\mathrm{AB}}$. This term is defined as the probability for finding a $\mathrm{B}$ atom at site $q$ after having found an A atom at site $p$ (Cowley, 1950). The probability for A-B pairs is $C_{\mathrm{A}} P_{p q}^{\mathrm{AB}}$ which must equal $C_{\mathrm{B}}$ $P_{p q}^{\mathrm{BA}}$, the number of B-A pairs. Also, ${ }_{p q}^{\mathrm{BB}}=1-P_{p q}^{\mathrm{BA}}, P_{p q}^{\mathrm{AA}}$ $=1-P_{p q}^{\mathrm{AB}} ; C_{\mathrm{A}}+C_{\mathrm{B}}=1$. With the Warren-Cowley definition of the short-range order (SRO) parameter (Cowley, 1950), $\alpha_{p q} \equiv 1-$ $P_{p q}^{\mathrm{AB}} / C_{\mathrm{B}}$. Spatial and time averages taken over the chemically distinct A-A, A-B, or B-B pairs with relative atom positions $p-$ $q$, produce the total elastically and quasielastic (thermal) scattered intensity in electron units for a crystalline solid solution of two components as

$$
\begin{aligned}
I(\mathbf{H})_{\text {Total }}= & \sum_{p} \sum_{q}\left[\left(C_{\mathrm{A}}^{2}+C_{\mathrm{A}} C_{\mathrm{B}} \alpha_{p q}\right)\left|f_{\mathrm{A}}\right|^{2}\left\langle e^{2 \pi i \mathbf{H} \bullet\left(\delta_{p}-\delta_{q}\right)}\right\rangle^{\mathrm{AA}}\right. \\
& +C_{\mathrm{A}} C_{\mathrm{B}}\left(1-\alpha_{p q}\right) f_{\mathrm{A}} f_{\mathrm{B}}^{*}\left(\left\langle e^{2 \pi \mathrm{H} \bullet\left(\delta_{p}-\delta_{q}\right)}\right\rangle^{\mathrm{BA}}\right. \\
& \left.+\left\langle e^{2 \pi i \mathbf{H} \bullet\left(\delta_{p}-\delta_{q}\right)}\right\rangle^{\mathrm{AB}}\right)+\left(C_{\mathrm{B}}^{2}+C_{\mathrm{A}} C_{\mathrm{B}} \alpha_{p q}\right)\left|f_{\mathrm{B}}\right|^{2} \\
& \left.\times\left\langle e^{2 \pi \mathrm{H} \boldsymbol{H}\left(\delta_{p}-\delta_{q}\right)}\right\rangle^{\mathrm{BB}}\right] \times e^{2 \pi i \mathbf{H}\left(\mathbf{R}_{p}-\mathbf{R}_{q}\right)}
\end{aligned}
$$

where $\left|f_{\mathrm{A}}\right|$ and $\left|f_{\mathrm{B}}\right|$ denote the absolute value or moduli of the complex amplitudes.

From the theoretical development given in Appendix A, a complete description of the diffusely distributed intensity through the second moment of the displacements is given as

$$
\frac{I(\mathbf{H})_{\text {Diffuse }}}{N}=\frac{I(\mathbf{H})_{\text {SRO }}}{N}+\frac{I(\mathbf{H})_{j=1}}{N}+\frac{I(\mathbf{H})_{j=2}}{N}
$$

where

$$
\begin{aligned}
\frac{I(\mathbf{H})_{\mathrm{SRO}}}{N} & =\sum_{l m n} C_{\mathrm{A}} C_{\mathrm{B}}\left|f_{\mathrm{A}}-f_{\mathrm{B}}\right|^{2} \alpha_{l m n} \cos \pi\left(h_{1} l+h_{2} m+h_{3} n\right) \\
\frac{I(\mathbf{H})_{j=1}}{N}= & -\operatorname{Re}\left[f_{A}\left(f_{\mathrm{A}}^{*}-f_{\mathrm{B}}^{*}\right)\right]\left[h_{1} Q_{x}^{\mathrm{AA}}+h_{2} Q_{y}^{\mathrm{AA}}+h_{3} Q_{z}^{\mathrm{AA}}\right] \\
& +\operatorname{Re}\left[f_{\mathrm{B}}\left(f_{\mathrm{A}}^{*}-f_{\mathrm{B}}^{*}\right)\right]\left[h_{1} Q_{x}^{\mathrm{BB}}+h_{2} Q_{y}^{\mathrm{BB}}+h_{3} Q_{z}^{\mathrm{BB}}\right]
\end{aligned}
$$

and

$$
\begin{aligned}
\frac{I(\mathbf{H})_{j=2}}{N}= & \left|f_{\mathrm{A}}\right|^{2}\left(h_{1}^{2} R_{X}^{\mathrm{AA}}+h_{2}^{2} R_{Y}^{\mathrm{AA}}+h_{3}^{2} R_{Z}^{\mathrm{AA}}\right) \\
& +f_{\mathrm{A}} f_{\mathrm{B}}^{*}\left(h_{1}^{2} R_{X}^{\mathrm{AB}}+h_{2}^{2} R_{Y}^{\mathrm{AB}}+h_{3}^{2} R_{Z}^{\mathrm{AB}}\right) \\
& +\left|f_{\mathrm{B}}\right|^{2}\left(h_{1}^{2} R_{X}^{\mathrm{BB}}+h_{2}^{2} R_{Y}^{\mathrm{BB}}+h_{3}^{2} R_{Z}^{\mathrm{BB}}\right) \\
& +\left|f_{\mathrm{A}}\right|^{2}\left(h_{1} h_{2} S_{X Y}^{\mathrm{AA}}+h_{1} h_{3} S_{X Z}^{\mathrm{AA}}+h_{2} h_{3} S_{Y Z}^{\mathrm{AA}}\right) \\
& +f_{\mathrm{A}} f_{\mathrm{B}}^{*}\left(h_{1} h_{2} S_{X Y}^{\mathrm{AB}}+h_{1} h_{3} S_{X Z}^{\mathrm{AB}}+h_{2} h_{3} S_{Y Z}^{\mathrm{AB}}\right) \\
& +\left|f_{\mathrm{B}}\right|^{2}\left(h_{1} h_{2} S_{X Y}^{\mathrm{BB}}+h_{1} h_{3} S_{X Z}^{\mathrm{BB}}+h_{2} h_{3} S_{Y Z}^{\mathrm{BB}}\right)
\end{aligned}
$$

Here the individual terms are defined in Appendix A. As illustrated in Equations 4, 5, 6, and 7, local chemical order (Warren-Cowley $\alpha$ 's) can be recovered from a crystalline binary alloy with a single contrast measurement of the diffuse scattering distribution, provided the displacement contributions are negligible. This was the early practice until a series of papers used symmetry relationships among the various terms to remove the $I(\mathbf{H})_{j=1}$ term in two dimensions (Borie and Sparks, 1964), in three dimensions (Sparks and Borie, 1965), and to second moment in all three dimensions: $I(\mathbf{H})_{\mathrm{SRO}}, I(\mathbf{H})_{j=1}, I(\mathbf{H})_{j=2}$ (Borie and Sparks, 1971), henceforth referred to as BS. The major assumption of the BS method is that the $\mathrm{x}$-ray atomic scattering factor terms $\left|f_{\mathrm{A}}-f_{\mathrm{B}}\right|^{2}, \operatorname{Re}^{*}\left[f_{\mathrm{A}}\left(f_{\mathrm{A}}^{*}-f_{\mathrm{B}}^{*}\right)\right]$, $\operatorname{Re}\left[f_{\mathrm{B}}\left(f_{\mathrm{A}}^{*}-f_{\mathrm{B}}^{*}\right)\right],\left|f_{\mathrm{A}}\right|^{2},\left|f_{\mathrm{B}}\right|^{2}$, and $f_{\mathrm{A}} f_{\mathrm{B}}^{*}$ of Equations $4,5,6$, and 7 have a similar $\mathbf{H}$ dependence so that a single divisor renders them independent of $\mathbf{H}$. With this assumption, the diffuse intensity can be written as a sum of periodic functions given by Equation 34. For neutron nuclear scattering, this assumption is excellent; neutron nuclear scattering cross-sections are independent of $\mathbf{H}$, and in addition, the TDS terms $\mathbf{C}$ and D can be filtered out. Even with $\mathrm{x}$ rays, the BS assumption is generally a good approximation. For example, as shown in Figure 4 for Mo $K_{\alpha} \mathrm{x}$ rays and even with widely separated elements such as Au$\mathrm{Cu}$, a judicious choice of the divisor allows the BS method to be applied as a first approximation over a large range in momentum transfer. In this case, division by $f_{\mathrm{Au}}\left(f_{\mathrm{Au}}-f_{\mathrm{Cu}}\right)=f_{\mathrm{Au}} \Delta f$ would be a better choice since the $\mathrm{Au}$ atom is the major scatterer. Iterative techniques to further extend the BS method have not been fully explored. This variation in the scattering factor terms with $\mathbf{H}$ has been proposed as a means to recover the individual pair displacements (Georgopoulos and Cohen, 1977).

Equations 5, 6, and 7 are derived from the terms first given by BS, but with notation similar to that used by Georgopoulos and Cohen (1977). There are 25 Fourier series in Equations 5, 6, and 7. For a cubic system with centrosymmetric sites, if we know $Q_{\mathrm{X}}^{\mathrm{AB}}$, then we know $R_{X}^{\mathrm{AA}}, R_{X}^{\mathrm{BB}}, R_{X}^{\mathrm{AB}}, S_{X Y}^{\mathrm{AA}}, S_{X Y}^{\mathrm{BB}}$, and $S_{X Y}^{\mathrm{AB}}$, then we know all the $Q, R$, and $S$ parameters. Thus with the addition of the $\alpha$ series, there are nine separate Fourier series for cubic scattering to second order.

As described in Appendix A (Derivation of the Diffuse Intensity), the nine distinct correlation terms from the $\alpha, \mathrm{Q}, \mathrm{R}$, and $\mathrm{S}$ series can be grouped into four unique $\mathbf{H}$-dependent functions, A, B, C, D within the BS approximation. By following the operations given by BS, we are able to recover these unique $\mathbf{H}$ dependent functions and from these the nine distinct correlation terms. For a binary cubic alloy, one x-ray map is sufficient to recover $\mathrm{A}, \mathrm{B}, \mathrm{C}$, and $\mathrm{D}$ and from $\mathrm{A}\left(h_{1} h_{2} h_{3}\right)$, the 
Warren-Cowley $\alpha$ 's. Measurements at two x-ray energies with sufficient contrast are required to separate the A-A and B-B pair contributions to the $\mathrm{B}\left(h_{1} h_{2} h_{3}\right)$ terms, and three $\mathrm{x}$-ray energies for the A-A, A-B, and B-B displacements given in Equation 29 and contained in the terms $\mathrm{C}$ and $\mathrm{D}$ of Equation 34 .

In an effort to overcome the assumption of $\mathbf{H}$ independence for the $\mathrm{x}$-ray atomic scattering factor terms and to use that information to separate the different pair contributions, Georgopoulos and Cohen (1977), henceforth GC, included the variation of the $\mathrm{x}$-ray scattering factors in a large least-squares program. Based on a suggestion by Tibballs (1975), GC used the $\mathbf{H}$ dependence of the three different $\mathrm{x}$-ray scattering factor terms to separate the first moment of the displacements for the A-A, A$\mathrm{B}$, and B-B pairs. Results from GC's error analysis (which included statistical, roundoff, x-ray scattering factors, sample roughness, and extraneous backgrounds) showed that errors in the x-ray atomic scattering factors had the largest effect, particularly on the $Q$ terms. They concluded, based on an error analysis of the BS method by Gragg et al. (1973), that the errors in the GC method were no worse than for the BS method and provided correct directions for the first moment displacements.

Improvements in the $\mathrm{GC}$ method, with the use of Mo $K_{\alpha} \mathrm{x}$ rays to obtain more data and the use of a Householder transformation to avoid matrix inversion and stabilization with ridge-regression techniques, still resulted in unacceptably large errors on the values of the $R$ and $S$ parameters (Wu et al., 1983). To date, there have been no reported values for the terms $R$ and $S$ that are deemed reliable. However, the Warren-Cowley $\alpha$ 's are found to have typical errors of $\sim 10 \%$ or less for binary alloys with a preference for unlike first-neighbor pairs with either the BS or GC analysis. For clustering systems, the BS method was reported to give large errors of $20 \%$ to $50 \%$ of the recovered $\alpha$ 's (Gragg et al., 1973). Smaller errors were reported on the $\alpha$ 's for clustering systems with the GC method (Wu et al., 1983). With increasing experience and better data from intense synchrotron sources, errors will be reduced for both the BS and GC methods.

Another methodology to recover the pair correlation parameters uses selectable x-ray energies (Ice et al., 1992). Of most practical interest are the $\alpha$ 's and the first moment of the static displacements as given in Equations 27 and 28. When alloys contain elements that are near one another in the periodic table, the scattering factor term $f_{\mathrm{A}}-f_{\mathrm{B}}$ can be made to be nearly zero by proper choice of $x$-ray energy nearby to an $x$-ray absorption edge. In this way, the intensities expressed in Equations 27 and 28 are made nearly zero and only that intensity associated with Equation 29 remains. Then, the term $I(\mathbf{H})_{j=2}$ can be measured with low scattering contrast between A and $\mathrm{B}$ atoms and after proper scaling, subtracted from diffuse scattering measurements made at other $\mathrm{x}$-ray energies (selected to emphasize the contrast between the $\mathrm{A}$ and $\mathrm{B}$ atoms). This leaves only the $I(\mathbf{H})_{\mathrm{SRO}}$ term, Equation 5, and the first moment of the static displacements $I(\mathbf{H})_{j=1}$, Equation 6. Shown in Figure 5 are the values of $\left|f_{\mathrm{Fe}}-f_{\mathrm{Cr}}\right|^{2}$ for x-ray energies selected for maximum contrast at $20 \mathrm{eV}$ below the $\mathrm{Fe} K$ and $\mathrm{Cr} K$ edges. The near null Laue energy, or energy of minimum contrast, was $7.6 \mathrm{keV}$.

The major assumption in this null Laue or $3 \lambda$ method is that the $I(\mathbf{H})_{j=2}$ and higher moment terms scale with x-ray Energy as $\left|C_{\mathrm{A}} f_{\mathrm{A}}+C_{\mathrm{B}} f_{\mathrm{B}}\right|^{2}$, which implies that the $\mathrm{A}$ and $\mathrm{B}$ atoms have the same second and higher moment displacements or that the different elements have the same $\mathrm{x}$-ray atomic scattering factors. This assumption is most valid for alloys of elements with similar atomic numbers, which have similar masses (similar thermal motion), atom sizes (small static displacement), and numbers of electrons (similar $\mathrm{x}$-ray scattering factors). This $3 \lambda$ method has been used to analyze five different alloys, $\mathrm{Fe}_{22.5} \mathrm{Ni}_{77.5}$ (Ice et al., 1992), $\mathrm{Cr}_{47} \mathrm{Fe}_{53}$ (Reinhard et al, 1992), $\mathrm{Cr}_{20} \mathrm{Ni}_{80}$ (Schönfeld et al., 1994), and $\mathrm{Fe}_{46.5} \mathrm{Ni}_{53.5}$ a recalculated $\mathrm{Fe}_{22.5} \mathrm{Ni}_{77.5}$ (Jiang et al., 1996), and $\mathrm{Cu}-\mathrm{Mn}$ (Schonfeld 2008). An improvement in the null Laue method by Jiang et al. (1996) removed an iteration procedure to account for the residuals left by the fact that $f_{\mathrm{A}}-f_{\mathrm{B}}$ was not strictly zero over the measured volume. The Cu-Mn study of Schonfeld found good agreement between the $3 \lambda$ and the GS methods.

The same techniques used for $\mathrm{x}$-ray diffuse scattering analysis can also be applied to neutron scattering measurements. Neutrons have the advantage (and complication) of being sensitive to magnetic order as described in Appendix B. This sensitivity to magnetic order allows neutron measurements to detect and quantify local magnetic ordering but complicates analysis of chemical ordering.

Error analysis of the null Laue method has been given by Jiang et al. (1995) and by Ice et al. (1998). The statistical uncertainties of the recovered parameters can be estimated by propagating the standard deviation $\pm \sqrt{n}$ of the total number of counts $n$ for each data point through the nonlinear least-squares processing of the data. Systematic errors can be determined by changing the values of input variables such as the $\mathrm{x}$-ray atomic scattering factors, backgrounds, and composition; then the data is reprocessed and the recovered parameters are compared.

Because the measured pair correlation coefficients are very sensitive to the relative and to a lesser degree the absolute intensity calibration of data sets collected with varying scattering contrast, the addition of constraints greatly increases reliability and reduces uncertainties. For example, the uncertainty in recovered parameters due to scaling of the measured scattering intensities is determined as input parameters are varied Each time, the intensities are rescaled so that the $I_{\mathrm{SRO}}$ values are everywhere positive and match values at the origin of reciprocal space measured by small-angle scattering. The integrated Laue scattering over a repeat volume in reciprocal space is also constrained to have an average value of $C_{\mathrm{A}} C_{\mathrm{B}}\left|f_{\mathrm{A}}-f_{\mathrm{B}}\right|^{2}$ (i.e., $\alpha_{000}=1$ ). These two constraints eliminate most of the systematic errors associated with converting the raw intensities into absolute units (Sparks et al., 1994). The intensities measured at three different energies are adjusted to within $\sim 1 \%$ on a relative scale and the intensity at the origin is matched to measured values. For these reasons, the systematic errors for $\alpha_{000}$ are estimated at $\sim 1 \%$.

For the null Laue method, errors on the recovered $\alpha$ 's and $\Delta X$ 's arising from statistical and various possible systematic errors in the measurement and analysis of diffuse scattering data are given in Tables 1 and 2 for the $\mathrm{Fe}_{46.5} \mathrm{Ni}_{53.5}$ alloy (Jiang et al., 1995; Ice et al., 1998). Details of the conversion to absolute intensity units are given elsewhere (Sparks and Borie, 1965; Ice et al., 1994; Warren, 1969; Reinhard et al., 1992). A previous assessment of the systematic errors, without the constraint of forcing $\alpha_{000}=1$ and keeping the intensity at the origin and fundamentals a positive match to known values, resulted in estimated errors approximately two to five times larger than those reported here (Jiang et al., 1995). Parameters necessary to the analysis of the data (other than well-known physical constants) with our best estimate of their standard deviations and their contributing standard deviations to the $\alpha$ and DX parameters are listed in Tables 1 and 2. From a comparison of theoretical and measured values, we estimate a 0.2 -eu error on the real part of the $\mathrm{x}$-ray atomic scattering factors, a $1 \%$ error in the $P_{0}$ calibration for converting the raw intensities to absolute units (eus), a 1-eu error in separating the inelastic resonant Raman scattering (RRS; Sparks, 1974), a 0- to 1-eu H dependent Compton scattering error (Ice et al., 1994), and an error of \pm 0.3 at.\% in composition (Ice et al., 1998). Systematic errors are larger than the statistical errors for the first three shells.

The asymmetric contribution of the first moment of the static displacements, $I_{j=1}$, Equation 13, to the diffuse intensity $I_{\mathrm{SRO}}+$ $\mathrm{I}_{j=1}$ for an $\mathrm{Fe}_{63.2} \mathrm{Ni}_{36.8}$ alloy is displayed in Figure 6. Without static displacements the $I_{\mathrm{SRO}}$ maxima would occur at the (100) and (300) superlattice positions. The static atomic displacements for the alloy are similar to those given in Table 2. Such large distortions of the short-range order diffuse scattering caused by displacements of $<0.02 \AA$ ( $0.002 \mathrm{~nm})$ emphasizes the sensitivity of this technique. With a change in the x-ray energy from 7.092 to $8.313 \mathrm{keV}, f_{\mathrm{Ni}}$ becomes smaller than $f_{\mathrm{Fe}}$. Figure 6 displays a reversal in the shift of the position of the diffuse scattering maxima. Two of these $x$-ray energies for the $3 \lambda$ method are chosen to emphasize this contrast and a third nearest the null Laue energy for removal of the TDS. The total estimated standard deviation on the values of the $\alpha$ 's and in particular the $\Delta X$ 's give unprecedented precision for the displacements with errors $\pm 0.003 \AA$ and less.

\section{PRACTICAL ASPECTS OF THE METHOD}

Local chemical order (Warren-Cowley $\alpha$ 's) from a crystalline binary alloy can be recovered with a single contrast measurement of the diffuse scattering distribution. Recovery of the two terms of the first moment of the static displacements $\left\langle\Delta X_{l m n}^{\mathrm{AA}}\right\rangle$ and $\left\langle\Delta X_{l m n}^{\mathrm{BB}}\right\rangle$ requires two measurements of 
sufficiently different contrast in $f_{\mathrm{A}} \Delta f^{*}$ and $f_{\mathrm{B}} \Delta f^{*}$ to separate those two contributions (Ice et al., 1992). Scattering contrast can be controlled in at least three ways: (1) by selecting x-ray energies near to and far from an absorption edge energy (resonance or anomalous x-ray scattering, Ice et al., 1994); (2) by measuring diffuse scattering over a wide $Q$ range where there is a significant change in the atomic form factors (Georgopoulos and Cohen, 1977); or (3) with neutrons by isotopic substitution (Cenedese et al, 1984).

The measurement of weak diffuse scattering normally associated with local order and displacements requires careful attention to possible sources of diffusely distributed radiation. Air scatter and other extraneous scattering from sources other than the sample, inelastic contributions such as Compton and resonant Raman, surface roughness attenuation, and geometrical factors associated with sample tilt must be removed. These details are important for placing the measured diffuse scattering in absolute units: a necessary requirement for the recovery of the $\alpha$ 's and displacements.

\section{Measurement of Diffuse X-ray Scattering}

Methods for collecting diffuse $\mathrm{x}$-ray scattering data from crystalline solid solutions have been discussed by Sparks and Borie (1965), Warren (1969), and Schwartz and Cohen (1987). As demonstrated in Equations 18 and 20, the x-ray scattering intensity from a solid solution alloy contains components arising from long-range periodicity of the crystalline lattice, correlations between the different atom types, and displacements of the atoms off the sites of the average lattice.

Because of the average periodicity of the crystalline solid solution lattice, the $x$-ray scattering repeats periodically in reciprocal space. The equations for recovering the various components are best conditioned when the data are collected in a volume of reciprocal space that contains at least one repeat volume for the diffuse scattering (Borie and Sparks, 1971). The volume required depends on the significance of the displacement scattering. If static displacements can be ignored and thermal scattering is removed, the smallest repeat volume for SRO is sufficient. This minimum volume increases as higher-order displacement terms become important, but in no case exceeds one-fourth of the volume bounded by $\Delta h_{1}=1, \Delta h_{2}=1$, and $\Delta h_{3}$ $=1$. A conservative approach is to measure the scattered intensity in a volume of reciprocal space that extends from the origin to as far out in $|\mathbf{H}|$ space as possible, but contains the minimum repeat volume for cubic symmetry. As illustrated in Figure 7 , the repeat volume for cubic crystals is $1 / 48$ of the reciprocal space volume limited by the maximum momentum transfer, $\sin \theta / \lambda=1 / \lambda$. This repeat unit contains all the accessible information about the structure of a crystal with average cubic symmetry. As it takes several days to prepare for the experiment and its setup, actual collection of the data is not the time-limiting step with $\mathrm{x}$ rays, and as much data as possible should be collected in an effort to accumulate 1000 or more counts at each of several thousand positions.

The various points $h_{1}, h_{2}, h_{3}$ in reciprocal space are measured by orienting the sample and detector arm with a four-circle diffractometer. Diffuse scattering data are typically collected at intervals of $\Delta h=0.1$ in a volume of reciprocal space bounded by $4 \geq h_{1} \geq h_{2} \geq h_{3} \geq 1$. Regions of detailed interest are measured at intervals of $\Delta h=0.05$. There are on the order of 7000 data points collected for a diffuse volume at each x-ray energy. By measuring diffuse scattering in a bisecting geometry where redundancy of the three sample-orienting circles is used to maintain the same incident and exit angle for the radiation with respect to the surface, the angular dependence of the absorption corrections is eliminated for theoretically-smooth surfaces and greatly reduced for rough surfaces (Ice 1994; Chukhovskii 2010).

Shown in Figure 8 is the typical optical train for the measurement of diffuse $x$-ray scattering. Every effort should be made to ensure that the detector receives the radiation from the sample with the same collection efficiency regardless of the sample orientation. The sample needs to be replaced with a wellcalibrated standard to convert the flux incident on the sample to absolute units. As all scattering measurements are made for fixed $I_{0}$ monitor counts, any changes to the optical train such as slit positions (excepting scatter slit) and sizes, distances, detectors, and changes in energy require replacing the sample with the scattering standard for recalibration of the incident flux against the $I_{0}$ monitor counts.

New challenges arise from the application of resonant (anomalous) x-ray scattering to the study of local order in crystalline solid solutions: (1) the need to work near absorption edges that can create large fluorescent and resonant Raman backgrounds, and (2) the need to know the resonant (anomalous) scattering factors and absorption cross-sections to $\sim 1 \%$, especially at $\mathrm{x}$-ray energies near absorption edges. Background problems due to inelastic scattering are exacerbated. Experimental measurement to recover the elastic scattering from these inelastic contributions (Compton, fluorescence, and resonant Raman) requires a combination of spectroscopy and diffraction.

\section{Removal of Inelastic Scattering Backgrounds}

Photoabsorption is the dominant $\mathrm{x}$-ray cross-section for elements with $Z>13$ and $\mathrm{x}$-ray energies $E<20 \mathrm{keV}$. The resultant fluorescence is typically orders of magnitude larger than the diffuse elastic scattering. Fluorescence can be removed by the use of an energy-sensitive detector when the incident $x$-ray energy is far enough above the photoabsorption threshold that the detector's energy resolution is adequate for separation.

Maximum change in scattering amplitude is obtained when measurements are made with the x-ray energy very near and then far from an absorption edge, as shown in Figure 9. The size of the $f^{\prime}$ component roughly doubles as the energy gap between the incident x-ray energy $E_{\mathrm{I}}$ and the absorption edge $E_{K}$ is halved. Near an edge, the size of the inelastic background grows rapidly due to RRS (Sparks, 1974; Eisenberger et al., 1976a, b; Åberg and Crasemann, 1994). RRS is interpreted as the filling of a virtual hole by a bound electron. As with fluorescence, the x-ray energy spectra is distinctive with peaks corresponding to the filling of the virtual hole, say in the $K$ shell, by various higher lying shells: $K$ filled from $L$, or $K$ filled from $M$ shells, often referred to as $K-L$ and $K-M$ RRS. Unlike fluorescence, the energy of the RRS peaks shift with incident $x$-ray energy, and the energy of the nearest RRS $K-L$ line is only a few tens of electronvolts from the incident $x$-ray energy (Åberg and Crasemann, 1994). This large inelastic background must either be removed experimentally or be calculated and subtracted.

The resolution of a solid-state detector at $\sim 150 \mathrm{eV}$ is inadequate to resolve all the RRS $K-M$ component from the elastic peak when excited near the threshold and can only resolve the Compton inelastic scattering at high scattering angles. A typical energy spectrum excited by $8.0-\mathrm{keV} \mathrm{x}$ rays on a $\mathrm{Ni}_{77.5} \mathrm{Fe}_{22.5}$ single crystal measured with a $\mathrm{Si}(\mathrm{Li})$ detector is shown in Figure 10. Near and below the Fe edge of $7.112 \mathrm{keV}$, the RRS $K-M$ component cannot be resolved from the elastic scattering peak. The RRS $K-M$ component can be removed by measuring the RRS $K-L$ component and assuming the $K-L K-M$ ratio remains constant. The Compton scattering component at low scattering angles is removed by using theoretical tables. Disadvantages to the use of solid-state detectors include the statistical and theoretical uncertainty of the inelastic contributions. Another disadvantage is the large deadtime imposed by the fluorescence $K$ signal in a solid-state detector, which restricts the useful flux on the sample.

Resolution on the order of 10 to $30 \mathrm{eV}$ is necessary to cleanly separate the resonant Raman and Compton scattering from elastic scattering. A crystal spectrometer improves the energy resolution beyond that available with a solid-state detector. Perfect crystal spectrometers are highly inefficient compared to mosaic crystal spectrometers due to their smaller integrated reflectivity. This inefficiency is unacceptable for typical weak diffuse $\mathrm{x}$-ray scattering. A mosaic crystal $\mathrm{x}$-ray spectrometer (Ice and Sparks, 1990) has been found to be a more practical device. The advantages of a mosaic crystal spectrometer is that it is possible to obtain energy resolutions similar to that of a perfect crystal spectrometer, but with an overall angular acceptance and efficiency similar to those of a solid-state detector. A schematic is shown in Figure 11A.

Figure 11B,C illustrates the ability of the graphite spectrometer to resolve Ni $K_{\alpha 1}$ from $K_{\alpha 2}$ and to resolve RRS from elastic scattering near the $\mathrm{Ni} K$ edge. Good efficiency is possible if the x-ray energy, $E_{\mathrm{I}}$, lies within a bandpass, $\Delta E$, set by the crystal Bragg angle $\theta_{\mathrm{B}}$ and the rocking curve width $\Delta \omega_{\mathrm{R}}$; $\Delta E=\Delta \omega_{\mathrm{R}} E \cot \theta_{\mathrm{B}}$. The diffracted beam is parafocused onto a 
linear detector and the beam position is correlated to $\mathrm{x}$-ray energy. The energy resolution and energy scale of the spectrometer are determined by varying the energy of the incident beam and observing the peak position of the elastic peak. At $8 \mathrm{keV}$, the bandpass, $\Delta E$, of a graphite crystal with a $0.4^{\circ}$ full-width at half-maximum (FWHM) mosaic spread is $\sim 250 \mathrm{eV}$. The energy resolution is limited by the effective source size viewed by the energy analyzer and by imperfections in the crystal. Energy resolutions of 10 to $30 \mathrm{eV}$ are typical with a 0.3 to $1.5-\mathrm{mm}$ high beam in the scattering plane at the sample.

Elastic scattering is resolved from the Compton scattering at higher scattering angles and $K-L$ RRS at all angles. When an xray energy is selected near but below the absorption edge of the higher $Z$ element, the lower $Z$ element will fluoresce. The mosaic crystal spectrometer can discriminate against this fluorescence and avoid deadtime from this emission. This graphite monochromator gives an overall decrease of 3 to 4 in counting efficiency compared with a solid-state detector, but provides a much cleaner signal with greatly reduced deadtime.

A consideration when using a crystal spectrometer is the sensitivity of the energy resolution to the effective source size. As the source size increases, the energy resolution decreases and increasingly small incident beams are required for good energy resolution (Ice and Sparks, 1990). In addition, the effective source size as viewed by the crystal spectrometer depends on the spread of the incident beam on the sample and the angle of the detector axis to the sample surface. These geometrical factors are governed by the scattering angle, $\theta$, and the chi tilt, $\chi$, as shown in Figure 12.

Diffuse scattering is normally collected in the bisecting mode as intended here. The size and shape of the beam intercept with the sample surface is determined by the beam height $h$, width $w$, and the sample angles $\theta$ and $\omega$. As shown in the insert of Figure 12, the intercept is a parallelogram that extends along the beam direction by $h / \sin \theta$ due to the beam height and $w \tan (\omega-$ $\pi / 2) / \sin \theta$ due to the beam width. In the reference frame of the detector, the length of the parallelogram is projected into a rootmeansquare source height of

$$
\sigma_{s}=\frac{\sqrt{[2 w \tan (\chi-\pi / 2) \cos \theta]^{2}+h^{2}}}{12}
$$

The measured energy resolution for a $1.5 \times 1.5-\mathrm{mm}^{2}$ beam at $\chi=55^{\circ}$ is plotted in Figure 12 as a function of scattering angle $\theta$. Deviations from $\chi=90^{\circ}$, where the sample normal lies in the scattering plane, are held to a minimum by choosing a crystal surface normal centered in the volume of reciprocal space to be measured. This reduces surface roughness corrections and maintains good energy resolution. Actual spectra collected during a diffuse scattering measurement are shown in Figure 13. Crystal analyzers will perform even better with third-generation synchrotron sources that have smaller source size and higher flux. Measurement of the fluorescent intensity or RRS throughout the volume used for the data with the identical optical train provides a correction for this beam spread, sample roughness, and alignment errors.

A model is used to describe the energy distributions of the RRS shown in Figures 13A,B as observed with the graphite spectrometer. The model contains a truncated Lorentzian centered at the energy for the RRS peak (Sparks, 1974; Ice et al., 1994). The high-energy cutoff is determined from energy conservation. The spectra are corrected for the graphite monochromator efficiency, which has a Gaussian distribution centered on the elastic scattering peak. The spectra are also corrected for the finite spectrometer resolution by convolving the Gaussian shape of the elastically scattered peak. The simple model for the resonant Raman peak shape allows for a good fit to the experimental resonant Raman peak observed with the graphite monochromator, as shown in Figures 13A,B.

Compton scattering can be removed from the elastic scatter by subtracting tabulated theoretical Compton intensities. It is possible to experimentally separate the elastic scattering peak from the Compton peak except at the lowest scattering angles. A correction for overlap of the two peaks at small angles is achieved by modeling the energy dependence of the Compton profile. The doubly differential Compton scattering cross-section is calculated using the impulse approximation (IA; Carlsson et al., 1982; Biggs et al., 1975). The Compton scattering is calculated for each subshell, and energy conservation is used to restrict the high-energy tail from each shell. The total spectrum is determined by adding the contribution from each shell and from each atom type. This cross-section is subtracted from the measured data normalized to absolute electron units per atom, which leaves only a resonant Raman peak and an elastic scattering peak. The slight overlap of the Compton peak with the elastic peak is typically small compared with statistical uncertainties.

Comparison of the calculated to the observed Compton spectrum can be achieved with $\mathrm{x}$-ray energies sufficiently removed from an edge that the resonant Raman contribution is negligible. At $8.000 \mathrm{keV}$, the resonant Raman contribution from an Fe-Ni sample is centered far below the elastic peak and outside the spectrometer window. The IA-calculated Compton profiles are observed to be in qualitative agreement with the observed spectra, but the intensity is overestimated at low angles and underestimated at high angles. Particularly noticeable is a low-energy tail at high scattering angles. A more exact theory without the impulse approximation might improve matters.

\section{Determination of the Resonant Scattering Terms $f^{\prime}$ and $f^{\prime \prime}$}

The widely used Cromer-Liberman tabulation (Cromer and Liberman, 1981; Sasaki, 1989) of $f^{\prime}$ and $f^{\prime \prime}$ explicitly ignores the presence of pre-edge unfilled bound states (bound-to-bound transitions), lifetime broadening of the inner-shell hole, and $\mathrm{x}$ ray absorption fine structure (XAFS; XAFS SPECTROSCOPY). These assumptions are justified $\sim 100 \mathrm{eV}$ below and $\sim 1 \mathrm{keV}$ above an edge, but not near an absorption edge (Lengeler, 1994; Chantier, 1994). Of particular concern is the underestimation of the absorption coefficient and $f^{\prime}$ just below an absorption edge due to the Lorentzian hole width of an inner shell. This is illustrated in Figure 14. An inner-shell hole with a 2-eV broadened lifetime and a $K$ edge jump ratio of 8 will increase the absorption cross-section by $\sim 11 \%$ at $20 \mathrm{eV}$ below the nominal edge and by $2 \%$ at $100 \mathrm{eV}$ below the edge. Theoretical tabulations that ignore the lifetime broadened hole width must be corrected, as they underestimate the absorption coefficient (and $f^{\prime}$ ) just below an edge and overestimate $f^{\prime \prime}$ above the edge. Theoretical tabulations also do not include unfilled pre-edge states or absorption fine structure that is highly sample dependent. Thus, it is necessary to determine the photoelectric absorption cross-section, $\mu / \rho$, experimentally for each sample, to calculate $f^{\prime \prime}$ with the optical theorem (James, 1948), and then to calculate $f^{\prime}$ from $f^{\prime \prime}$ with the Kramers-Kronig relationship.

The practical method of measuring the sample-specific absorption cross-section is to measure the relative absorption cross-section across the edge of each of the elements of the sample over a range of $\sim 100$ to $1000 \mathrm{eV}$. These data are normalized to theoretical data far from the edge (Kawamura and Fukamachi, 1978; Dreier et al., 1983; Hoyt et al., 1984). Measurements are made with a thin foil of the sample in transmission geometry. The measured value of $f^{\prime}$ is obtained by adding the difference integration to tabulated values of $f^{\prime}$, as shown in Figure 9.

We find $f^{\prime}$ is 5 to 10 times less sensitive than $f^{\prime \prime}$ to lifetime broadening of the inner-shell hole. For example, the effect of a 2-eV lifetime on $f^{\prime}$ at $20 \mathrm{eV}$ below the edge is only $2 \%$ and at $-100 \mathrm{eV}$ the effect is only $0.3 \%$. The value of $f^{\prime}$ is sensitive to the position of the average inflection point of the absorption edge. A shift of $5 \mathrm{eV}$ results in a $4 \%$ to $5 \%$ change in $f^{\prime}$ at $20 \mathrm{eV}$ below the edge. Errors in the absolute energy calibration are removed when the energy of the incident radiation is fixed to the same absorption edge energy as the calculation of $f^{\prime}$ and $f^{\prime \prime}$.

As shown in Figure 15A,B, the absorption edge energies for $\mathrm{Fe}$ and $\mathrm{Ni}$ in a fully ordered $\mathrm{FeNi}_{3}$ foil do not shift compared to the absorption edge energies of pure $\mathrm{Fe}$ or Ni foils. However, the local environment of the $\mathrm{Fe}$ atoms in $\mathrm{FeNi}_{3}$ are sufficiently different that calculated or measured values of $f^{\prime}$ and $f^{\prime \prime}$ for pure $\mathrm{Fe}$ would be in error close to the $\mathrm{Fe}$ absorption edge. For samples where there is a large charge transfer (change in oxidation state), this difference becomes even larger.

\section{Absolute Calibration of the Measured Intensities}

Conversions to absolute units depend on previously calibrated standards to place the intensity in electron units (Suortti et al., 1985). Calibrated powder standards account for the monitor efficiency, the beam path transmission, and the efficiency and 
solid angle of the detector. The largest uncertainty is in the values of the linear x-ray absorption cross-sections, $\mu$, near an absorption edge for both the powder and the sample. This problem is reduced by using a powder similar in elemental composition to the sample or by careful calibration of the sample absorption. Comparison between standardizations with various powder samples are consistent to within 3\% to 5\% (Suortti et al., 1985).

The relative scaling factors between different energy sets can be refined with great sensitivity by restricting the short-rangeorder intensity as discussed previously and as described below. For alloys that cluster, the Laue scattering can be obscured by proximity of the fundamental Bragg peaks. Higher-resolution measurements such as small-angle x-ray scattering (SAXS) techniques may then be required to recover the Laue scattering. SAXS can be used to measure the total SRO scattering at the origin, and the relative scaling factors of data sets can be adjusted so that $\alpha_{000}=1$, and the fitted SRO diffuse scattering, approach the SAXS value obtained near the origin. This scaling method is illustrated in Figure 16 for a $\mathrm{Ni}_{77.5} \mathrm{Fe}_{22.5}$ alloy. As shown in Figure 16, a small change in the relative scaling (here the zero contrast or near null Laue scale factor) makes a big change in the SRO scattering near the origin. Fe-Ni alloys are known to show negligible scattering near the origin (Simon and Lyon, 1991). Scale factors are adjusted to set the SRO scattering to near zero at the Bragg peaks. Nonlinear fitting routines that refine the relative intensities and include these and other restrictions may be possible with more reliable data sets from third-generation synchrotron sources.

\section{DATA ANALYSIS AND INITIAL INTERPRETATION}

Most x-ray detectors are count-rate limited, and measured x-ray scattering intensities must be corrected for detectorsystem deadtime (Ice et al., 1994). With proportional counters and solidstate detectors, the measured deadtime is typically 3 times the amplifier shaping constant. For most measurements, this results in deadtimes of $\sim 1$ to $10 \mu \mathrm{s} /$ count

Detector survival can also be challenged by intense x-ray beams and requires that the sample orientation and detector position be controlled such that a Bragg reflection from the cyrstal does not enter the detector. Bragg reflections can contain in excess of $10^{9} \mathrm{x}$ rays per second at synchrotron sources, which can paralyze or damage detectors. Position-sensitive wire proportional counters are especially vulnerable, and count rates below $10^{4}$ counts/s are generally advisable to prevent damage to the wire or coated filament. Just flashing through a Bragg reflection when changing orientation can damage the wire anode of a linear-senitive proportional counter, and materially degrades its spatial resolution. Extreme caution is necessary when measurements are taken near Bragg reflections with fluxsensitive detectors.

Mirrors and crystal monochromators can pass a significant number of harmonics (multienergy $\mathrm{x}$ rays), which are then diffracted by the sample at positions of $h / n, k / n, l / n$, where $n$ is an integer and $h k l$ are the Miller indices of the Bragg reflections. Any sharp spikes observed in the measurement of diffuse intensities at these positions are suspect and must be removed before processing the data to recover the local correlations. At the position (100) in Figure 17, we note an outlying data point that can be attributed to the Bragg diffraction from the (200) reflection of an x-ray energy twice that of the nominal energy. Such spurious data can also be caused by surface films left from chemical treatment or oxidation.

An example of the raw data measured for three different $\mathrm{x}$ ray energies from an $\mathrm{Fe}_{46.5} \mathrm{Ni}_{53.5}$ alloy single crystal is shown in Figure 17 (Jiang et al., 1996). The solid line in Figure 17 is the near null Laue measurement, which can be used to remove the quadratic and higher-order displacement terms from the other data sets. The assumption is made that for elements with similar masses and small static displacements the second and higher moment terms are the same for both atoms species so that for $I_{j>2}$, the intensity scales as $\langle f\rangle^{2}$ This method avoids the need to calculate thermal scattering from a set of force constants with the Born-von Karman central forces model that assumes harmonic vibrations and Hooke's law forces between the atoms independent of their environment (Warren, 1969). Theoretically calculated TDS increasingly deviates from measured TDS on approach to the Brillouin zone boundaries. This is not unexpected as local structure is important near the zone boundaries. A comparison of x-ray null Laue results with neutron diffuse scattering measurements, which are not complicated by TDS, gives very similar $\alpha$ 's for $\mathrm{Fe}_{3} \mathrm{Ni}$ (Jiang et al., 1996).

An example of the measured diffuse scattering data in the $h_{1} h_{2} 0$ plane (labeled here as $\mathrm{H}_{1}, \mathrm{H}_{2}, 0$ ) for three $\mathrm{x}$-ray energies is shown in Figure 18 for an alloy of $\mathrm{Fe}_{27.5} \mathrm{Ni}_{77.5}$ (Ice et al, 1992). The x-ray energy at $8.000 \mathrm{keV}$ (Fig. 18B) is the near null Laue energy $\left(f_{\mathrm{Ni}}-f_{\mathrm{Fe}} \sim 0\right)$, where $I_{\mathrm{SRO}}$ is nearly zero compared with the intense SRO maxima such as (100), (110), and (210) at 7.092 $\mathrm{keV}$ of Figure 18A. With the data in electron units and with inelastic scattering removed, the data are now processed to recover the $\alpha$ and $\delta$ values. As discussed previously (see Principles of the Method), we have the choice of different processing methods.

1. The null Laue method (also referred to as the $3 \lambda$ method). This method is used when the elements of an alloy are sufficiently near each other in the periodic table that their dynamic displacements are similar and $I_{j \geq 2}$ scales as $\left(C_{\mathrm{A}} f_{\mathrm{A}}+C_{\mathrm{B}} f_{\mathrm{B}}\right)^{2}$ for the different energies; $I_{>>2}$ can be experimentally measured at a low x-ray scattering contrast and then substracted from the diffuse scattering with high elemental contrast (Ice et al., 1992; Reinhard et al., 1992; Schoenfield et al, 1994; Jiang et al, 1996). The null Laue method is implemented using a nonlinear least-squares approach.

2. Collection of sufficient data such that the BS separation technique can first be used to recover $I_{\mathrm{SRO}}, I_{j=1}, I_{j=2}$, and higher terms separately for each of the three $\mathrm{x}$-ray energies. Then a least-squares program is used to recover the $\alpha$ 's from $I_{\mathrm{SRO}}$ The $\alpha$ 's are used to recover the displacements from $I_{j=1}$ and the second moments from $I_{j=2}$ as given in Equations 4, 5, 6 , and 7. As the assumption is made that the x-rayatomic scattering factors are independent of $\mathbf{H}$, aninteractive technique is required to remove that assumption. This is possibly the most robust of the methods as it does not have the assumption of method 1 . Therefore it can be extended to include the $I_{j=3}$ and $I_{j=4}$ terms to account for higher moments of the displacements including second-order TDS, which becomes important for measurements made at temperatures approaching the Debye temperature. Furthermore, data for each energy can be separately into $I_{\mathrm{SRO}}$ and displacement scattering and checked for the correct normalization factors before different energy sets are subjected to a simultaneous least squares program to recover the A-A: A-B, and B-B pair correlations.

3. The GC method of analysis, which uses data measured at only one energy for 25 symmetry-related points for each of the 25 terms expressed in Equations 4, 5, 6, and 7. These symmetryrelated points are chosen such that the values of $I_{\mathrm{SRO}}$, and the $Q, R$, and $S$ terms are of the same or of opposite magnitude. Only their scattering factors and $\mathbf{H}$ dependence differ. In this way, the terms of Equations 4, 5, 6, and 7 are obtained from a system of linear equations stabilized by a ridge regression technique. These terms are then inverted to recover their Fourier coefficients. Results with this technique have been mixed. An analysis of $\mathrm{AuCu}_{3}$ data (Butler and Cohen, 1989) concluded that the $\mathrm{Au}-\mathrm{Au}$ bond distance was shorter than that for $\mathrm{Cu}-\mathrm{Cu}$. This result is contrary to the experimental findings that ordering of $\mathrm{AuCu}_{3}$ reduces the lattice constant as more first-neighbor $\mathrm{Au}-\mathrm{Cu}$ pairs are formed and that the addition of the $\sim 14 \%$ larger $\mathrm{Au}$ atoms to $\mathrm{Cu}$ increases the lattice constant because of the larger $\mathrm{Au}-\mathrm{Au}$ bond distance. Theoretical considerations have also concluded that the $\mathrm{Au}-\mathrm{Au}$ bond distance is the largest of the three kinds (Chakraborty, 1995; Horiuchi et al., 1995). Apparently, the $\mathbf{H}$ variation of $f_{\mathrm{Au}} \Delta f^{*}$ and $f_{\mathrm{Cu}} \Delta f^{*}$ shown in Figure 4 is not sufficiently different to provide for a meaningful separation of the $\mathrm{Au}-\mathrm{Au}$ and $\mathrm{Cu}-\mathrm{Cu}$ bond distances. In a direct comparison with the $3 \lambda$ technique on an alloy of $\mathrm{Ni}_{80} \mathrm{Cr}_{20}$, the GC result gave a $\mathrm{Ni}-\mathrm{Ni}$ bond distance with a different sign, which was contrary to other information (Schöenfeld et al., 1994). In addition, published GC values for $\left\langle(\Delta X)^{2}\right\rangle$ coefficients are not reliable (Wu et al., 1983). Though first-order TDS is included in the separation, higher-order TDS is calculated from force constants and subtracted.

Interpretation of Recovered Static Displacements 
The displacements are defined as deviations from the average lattice and are given by

$$
\mathbf{r}_{p}-\mathbf{r}_{q}=\left(\mathbf{R}_{p}-\mathbf{R}_{q}\right)+\left(\delta_{p}-\delta_{q}\right)
$$

As we can move the frame of reference so that its origin always resides on one of the atoms of the pair, such that $\mathbf{r}_{P} \equiv \mathbf{r}_{0}, \mathbf{R}_{p} \equiv$ $\mathbf{R}_{0}$, and $\delta_{p} \equiv \delta_{0}$, then

$$
\mathbf{r}_{p}-\mathbf{r}_{q}=\mathbf{r}_{0}-\mathbf{r}_{q}=\mathbf{r}_{0}-\mathbf{r}_{l m n}=-\mathbf{r}_{l m n}
$$

and with the atom pair identified by $i j$

$$
\mathbf{r}_{l m n}^{i j}=\mathbf{R}_{l m n}+\boldsymbol{\delta}_{l m n}^{i j}
$$

where $\mathbf{R}_{l m n}$ is independent of the kinds of atom pairs since it is defined by the average lattice (i.e., Bragg reflection positions). The average value of the measured $\mathbf{r}_{l m n}$ for all the $N$ pairs contributing to the measured intensity is

$$
\left\langle\mathbf{r}_{l m n}^{i j}\right\rangle=\frac{1}{N^{i j}} \sum_{i j}\left\langle\mathbf{R}_{l m n}+\delta_{l m n}^{i j}\right\rangle=\mathbf{R}_{l m n}+\left\langle\delta_{l m n}^{i j}\right\rangle
$$

Here $\left\langle\delta_{l m n}^{i j}\right\rangle$ is the variable recovered from the diffuse scattering. As shown in Equation 25, we recover the Cartesian coordinates of the average displacement vector,

$$
\left\langle\delta_{l m n}^{i j}\right\rangle \equiv\left\langle\Delta X_{l m n}^{i j}\right\rangle \mathbf{a}+\left\langle\Delta Y_{l m n}^{i j}\right\rangle \mathbf{b}+\left\langle\Delta Z_{l m n}^{i j}\right\rangle \mathbf{c}
$$

For cubic systems, when the atom has fewer than 24 neighboring atoms in a cogrdination shell (permutations and combinations of $\pm l, \pm m, \pm n),\left\langle\delta_{l m n}^{i j}\right\rangle$ must be parallel to the lattice vector $\mathbf{R}_{l m n}$. This maintains the statistically observed long-range cubic symmetry even though on a local scale this symmetry is broken. For Imn multiplicities $\geq 24$, the displacements on average need not be parallel to the average interatomic vector $\mathbf{R}_{l m n}$ to preserve cubic symmetry (Sparks and Borie, 1965).

Measurements of diffuse scattering from single crystals provides the components of the atomic displacements $(\Delta X)$, $(\Delta Y)$, and $(\Delta Z)$ whereas the spherical average usually obtained from EXAFS and $x$-ray measurements on amorphous materials and crystalline powders gives only the magnitude of the radial displacements. Thus, diffuse x-ray scattering from single crystals provides new information about the vector displacements associated with near-neighbor chemistry.

Measured displacements such as those presented in Table 2 provide unique insight into how atoms move off their lattice sites when local symmetry is broken. Local symmetry is broken when a multicomponent crystalline material is above the ordering temperature (with less-than-perfect long-range order) and/or off stoichiometry. With perfect long-range order the atoms are constrained to lie precisely on the sites of the average lattice by balanced forces. In alloys, where the local symmetry is broken, we gain new insights into the chemically distinct bonding, including the interatomic bond distances and whether the displacements have both radial and tangential components. With reference to Figure 23, the displacement for the [110] nearestneighbor atoms is on average radial with a magnitude given by $\left|\left\langle\delta_{110}\right\rangle\right|=\sqrt{2}\left|\Delta X_{110}\right|$.

We note that the $\mathrm{Fe}-\mathrm{Fe}$ first-neighbor pair distances given in Table 2 are 0.021(3) $\AA \times \sqrt{2}=0.030$ (4) $\AA$ further apart then the average lattice and that second neighbor pairs are closer by (-) 0.023(1) $\AA$. Average bond distances along the interatomic vector between nearest-nejghbor pairs for this fcc lattice are obtained by adding the $\sqrt{2}\left|\Delta X_{110}\right|$ to the average interatomic vector $\mathbf{R}_{110}$, as defined in Figure 19. The parameter $\left|\mathbf{R}_{110}\right|$ is just the cubic lattice constant lal times $1 / \sqrt{2}$. From the construction shown in Figure 19, it follows that the vector distance between a pair of atoms, $\mathbf{r}_{l m n}^{i j}$, has radial and tangential displacement components with magnitudes given by

$$
\left|\delta_{l m n}^{i j}\right|_{\square}=\frac{\delta_{l m n}^{i j} \bullet \mathbf{R}_{l m n}}{\left|\mathbf{R}_{l m n}\right|}
$$

and

$$
\left|\delta_{l m n}^{i j}\right|_{\perp}=\sqrt{\left|\delta_{l m n}^{i j}\right|^{2}-\left|\delta_{l m n}^{i j}\right|_{\square}^{2}}
$$

The radial (II) and tangential $(\perp)$ components of the displacements recovered from diffuse scattering measurements on single crystals are shown in Figure 19.
As the $\mathrm{Fe}_{46.5} \mathrm{Ni}_{53.5}$ alloy is cubic (face centered), the $\Delta Y$ and $\Delta Z$ displacements are derived from the $\Delta X$ 's given in Table 2 by permutation of the indices. (Henceforth, we will omit the $<>$ on the displacements for simplicity.) For example, $\Delta X_{321}$ has the identical value as $\Delta Y_{231}$ and $\Delta Z_{123}$, and $\Delta X_{321}=\Delta X_{312}=\Delta Y_{231}=$ $\Delta Y_{132}=\Delta Z_{123}=\Delta Z_{213}$. In addition, $\Delta X_{321}=-\Delta X_{321}$ and similarly for the other combinations as illustrated in Figure 20. The nearest-atom pairs that could have, on average, nonradial components are those in the third neighboring shell, $\operatorname{lm} n=211$ (Sparks and Borie, 1965). If the displacements between atom pairs is on average along their interatomic vector, then $\Delta X_{211}=2$ $\Delta X_{121}$ (Fig.20). For the Fe-Fe pair displacements given in Table $2, \Delta X_{211}=0.0005(2) \AA$ and $2 \Delta X_{121}=0.0028(8) \AA$; thus the (211) $\mathrm{Fe}-\mathrm{Fe}$ pair displacements have a significant tangential component. From Equations 14 and 15, the magnitude of the displacement between (211) $\mathrm{Fe}-\mathrm{Fe}$ pairs along the radial direction $\left|\delta_{211}^{\mathrm{Fe}-\mathrm{Fe}}\right|_{||}$is $0.0016(7) \AA$ and $\left|\delta_{211}^{\mathrm{Fe}-\mathrm{Fe}}\right|_{\perp}$ tangential is $0.0013(7) \AA$. Thus the (211) Fe-Fe neighbors have a similar radial and tangential component to their displacements. For the (310) Fe-Fe pair displacements, $\Delta X_{310} \cong 3 \Delta X_{130}$ within the total estimated error, and on average (310) displacements are predominantly radial. These measured displacements provide new information not obtained in other ways about the local atomic arrangements in crystalline solid solutions.

Results for the few crystalline binary alloys that have had their individual pair displacements measured with this $3 \lambda$ technique are summarized in Figure 21. Here the $\Delta X$ static displacements are plotted as a function of the radial distance $\frac{1}{2} \sqrt{l^{2}+m^{2}+n^{2}}$. When there is more than one value for $\Delta X$, the plots show the various values. Most striking is the observation that for the three ordering alloys the near- neighbor $\mathrm{Fe}-\mathrm{Ni}$ and $\mathrm{Cr}-\mathrm{Ni}$ bond distances are the smallest of the three possible pairs (Fig. 21). However, for the clustering $\mathrm{Cr}_{47} \mathrm{Fe}_{53}$ alloy the $\mathrm{Cr}-\mathrm{Cr}$ nearest-neighbor bond distances are closest and the $\mathrm{Cr}-\mathrm{Fe}$ are furthest apart. More details, including the short-range-order parameters $\alpha$ and numerical values of the displacements for each shell, are given in the original papers. These pair displacement observations provide a more rigid test of theoretical predictions than variations of the average lattice parameter with concentrations (Froyen and Herring, 1981; Mousseau and Thorpe, 1992).

\section{METHOD AUTOMATION}

Because of the large data sets collected ( 7000 data points per diffuse scattering volume, each consisting of a multichannel energy spectrum with 200 to 500 channels), data collection is under computer control. The program most widely used for converting the reciprocal space coordinates to Eulerian angles and then stepping the three- (or four-) axis diffractometer to this list of coordinates is SPEC (Certified Scientific Software). Because the diffuse scattering is a slowly varying function without sharp peaks, it is fastest to take the data while the diffractometer is moving and avoid the time-consuming starting and stopping of the diffractometer. The reciprocal space coordinate is estimated from the midrange of the angular scans during data collection. When using the BS separation procedure, it is necessary to have the diffuse scattering data on a uniform cubic grid in reciprocal space. Least-squares procedures do not have this requirement. Since the experiment is enclosed in a radiation hutch, computerized control of all slits, sample positioning and electronic components in the hutch is desirable.

\section{SAMPLE PREPARATION}

The most detailed measurements of diffuse x-ray scattering are made on homogeneous single crystals with polished and etched surfaces. These samples must be carefully prepared and handled to minimize surface roughness effects, surface contamination, and inhomogeneities. To prepare a sample, a single crystal of the intended composition and $\geq 99.9 \%$ purity metals should be homogenized for $\geq 3$ days at $\sim 50^{\circ}$ to $100^{\circ} \mathrm{C}$ below the crystal melting point in an atmosphere that protects its composition. This step is intended to create a single crystal with uniform chemical composition. For a crystal with cubic symmetry, the crystal should be oriented and then cut with a surface near the $<421>$ normal. This orientation minimizes the goniometer $\chi$ range, improves the energy resolution of measurements with a crystal spectrometer, and improves access to a cubic symmetry volume of reciprocal space. The surface of the crystal should be 
$\sim 20 \mathrm{~mm}$ in diameter to ensure that the incident beam is completely intercepted by the sample at all orientations. Electrical discharge machining or chemical sawing minimizes surfaces strains and distortions and is preferred to standard machining. After chemical polishing to remove distorted metal, the crystals should be heat treated to the desired state of thermodynamic equilibrium before quenching. Metallographic polishing is followed by chemical dissolution or electropolishing to produce a mirror smooth surface with nondistorted metal (see SAMPLE PREPARATION FOR METALLOGRAPHY). Verification of negligible distortion is obtained by optical microscopy of subgrain boundary images. Because surface roughness reduces $\mathrm{x}$-ray scattering intensity at low angles, smooth surfaces reduce the corrections to the absolute intensity measurements. The effect of sample roughness on intensity can be checked with fluorescence or RRS measurements to determine if there is any angular variation with respect to $\mathbf{H}$ in the bisecting geometry (Sparks et al., 1992). The acceptable mosaic spread of the crystals measured from x-ray rocking curves depends on the $\mathbf{H}$ resolution necessary to resolve the features of interest, but can be as large as $\sim 2$ degrees. Verification of the composition to \pm 0.2 at.\% or less is needed to reduce the uncertainty in the data analysis. A check of the weight of the raw materials against the weight of the single-crystal ingot will determine if more extensive chemical analysis is necessary.

\section{PROBLEMS}

The fundamental problem of diffuse x-ray scattering is that the measurements must be made in absolute units and must be consistent over large ranges in reciprocal space and with different experimental conditions (x ray, neutron, energy, etc.). Even small systematic uncertainties increase the uncertainties in the recovered local correlations. Special procedures can sometimes be used to calibrate the relative normalization between different experimental conditions, but the reality remains that great care is required to collect meaningful data. For example, near an absorption edge, uncertainties in the scattered intensity can occur because the values of $f^{\prime}$ and $f^{\prime \prime}$ change rapidly with small fluctuations in incident photon energy, as seen in Figure 9. The intensities of RRS also increases as the incident photon energy approaches an absorption edge. As a compromise, the incident energies are usually chosen $\sim 20 \mathrm{eV}$ below an absorption edge. This requires that the $\mathrm{x}$-ray optics, which select the x-ray energy from the white synchrotron spectrum, be very stable so as to control the selected energy fluctuations to less than about $\pm 1 \mathrm{eV}$.

Where limited information is acceptable, problems of slow data collection and difficulties in systemmatic errors associated with measurments over large volumes of reciprocal space can be minimized. For example, measurements of intensity and peak width for a single superstructure reflection can be used to monitor correlations lengths and tendencies during rearrangements of local atomic coordinations (Mudgel M 2007).

Similarly, environments must maintain a clean surface free of condensates and oxidation that can add unwanted scattered intensity to measurements. Extremely thin coverages of a few tens of monolayers can contribute noticeable intensity. Equilibrium temperatures can sometimes be difficult to define on quenched samples. For example, quenching to a desired equilibrium local-ordered state may not be possible due to rapid kinetics; quenched-in vacancies enhance diffusion, which can alter chemical order at the actual temperature of interest for local order. Kinetic studies or measurements at the higher temperature are required to ensure that equilibrium order is achieved. Even if diffusion, which changes the $\alpha$ 's on cooling, is not a problem, changes in lattice constant on cooling may affect the static displacements. It is better to have the sample in a known state of equilibrium so that the recovered parameters can be directly compared to theory. A discussion of the effect of quenching parameters on the diffuse scattering from an $\mathrm{Al}-\mathrm{Cu}$ sample has been given by Epperson et al. (1978), and a study of the kinetics of short-range ordering in $\mathrm{Ni}_{0.765} \mathrm{Fe}_{0.235}$ has been given by Bley et al. (1988).

The complexities of actually performing and interpreting a three-dimensional diffuse scattering experiment require a major commitment of time and resources. To ensure success, we suggest that beginners collaborate with one of the referenced authors who has experience in this science and who has access to the specialized instrumentation and software required for successful diffuse scattering experiments.

\section{ACKNOWLEDGMENTS}

We wish to express our appreciation to the early pioneers of diffuse scattering and to our many contemporaries who have contributed to this subject. Research was sponsored by the U.S. Department of Energy, Basic Energy Sciences, Materials Sciences and Engineering Division.

\section{LITERATURE CITED}

Åberg, T. and Crasemann, B. 1994. Radiative and radiationless resonant Raman scattering. In Resonant Anomalous X-Ray Scattering: Theory and Applications (G. Materlik, C. J. Sparks, and F. Fischer, eds.). pp. 431-48. Elsevier Science Publishing, New York.

Biggs, F., Mendelshohn, L. B., and Mann, J. B. 1975. HartreeFock Compton profiles for the element. Atomic Data Nucl. Tables 16:201-309.

Bley, F., Amilius, Z., and Lefebvre, S. 1988. Wave vector dependent kinetics of short-range ordering in ${ }^{62} \mathrm{Ni}_{0.765} \mathrm{Fe}_{0.235}$, studied by neutron diffuse scattering. Acta Metall. Mater. 36:1643-1652.

Borie B. and Sparks C. J. 1964. The short-range structure of copper-16 At. \% aluminum. Acta. Crystallogr. 17: 827-835.

Borie, B. and Sparks, C. J. 1971. The interpretation of intensity distributions from disordered binary alloys. Acta Crystallogr. A27:198-201.

Butler, B. D. and Cohen, J. B. 1989. The structure of $\mathrm{Cu}_{3} \mathrm{Au}$ above the critical temperature. J. Appl. Phys. 65:2214-2219.

Carlsson, G. A., Carlsson, C. A., Berggren, K., and Ribberfors, R. 1982. Calculation of cattering cross sections for increased accuracy in diagnostic radiology. 1. Energy broadening of Compton-scattered photons. Med. Phys. 9:868-879.

Cenedese, P., Bley, F., and Lefebvre, S. 1984. Diffuse scattering in disordered ternary alloys: Neutron measurements of local order in stainless steel $\mathrm{Fe}_{0.56} \mathrm{Cr}_{0.21} \mathrm{Ni}_{0.23}$. Acta Crystallogr. A40:228-240.

Chantier, C. T. 1994. Towards improved form factor tables. In Resonant Anomalous X-Ray Scattering: Theory and Applications (G. Materlik, C. J. Sparks, and K. Fischer, eds.). pp. 61-78. Elsevier Science Pubishers, New York.

Chakraborty, B. 1995. Static displacements and chemical correlations. Eur. Phys. Lett. 30:531-536.

Chukhovskii, F. N., Polyakov, A. M. 2010. X-ray Specular Scattering From Statistically Rough Surfaces: A Novel Theoretical Approach Based on the Green Function Formalism, Acta Crystallogr. A66:640-648.

Cowley, J. M. 1950. X-ray measurement of order in single crystals of $\mathrm{Cu}_{3} \mathrm{Au}$. J. Appl. Phys. 21:24-30.

Cromer, D. T. and Liberman, D. 1970. Relativistic calculations of anomalous scattering factors for $\mathrm{x}$ rays. J. Chem. Phys. 53:1891-1898.

Cromer, D. T. and Liberman, D. 1981. Anomalous dispersion calculations near to and on the long-wavelength side of an absorption edge. Acta. Crystallogr. A37:267-268.

Dreier, P., Rabe, P., Malzfeldt, W., and Niemann, W. 1983. Anomalous scattering factors from $\mathrm{x}$-ray absorption data by Kramers-Kronig analysis. In Proceedings of the International Conference on EXAFS and Near Edge Structure, Frascati, Italy, Sept. 1982 (A. Bianconi, L. Incoccia, and S. Stipcich, eds.). pp. 378-380. Springer-Verlag, New York and Heidelberg.

Drijver, J. W., van der Woude, F., and Radelaar, S. 1977. Mössbauer study of atomic order in $\mathrm{Ni}_{3} \mathrm{Fe}$. I. Determination of the long-range-order parameter. Phys. Rev. B 16:985-992.

Eisenberger, P., Platzman, P. M., and Winick, H. 1976a. X-ray resonant Raman scattering: Observation of characteristic radiation narrower than the lifetime width. Phys. Rev. Lett. 36:623-625.

Eisenberg, P., Platzman, P. M., and Winick, H. 1976b. Resonant $\mathrm{X}$-ray Raman scattering studies using synchrotron radiation. Phys. Rev. B. 13:2377-2380.

Epperson, J. E., Fürnrohr, P., and Ortiz, C. 1978. The shortrange-order structure of $\alpha$-phase $\mathrm{Cu}-\mathrm{Al}$ alloys. Acta Crystallogr. A34:667-681.

Froyen, S. and Herring, C. 1981. Distribution of interatomic spacings in random alloys. J. Appl. Phys. 52:7165-7173. 
Gehlen, P. C. and Cohen, J. B. 1965. Computer simulation of the structure associated with local order in alloys. Phys. Rev. A 139:884-A855.

Georgopoulos, P. and Cohen, J. B. 1977. The determination of short range order and local atomic displacements in disordered binary solid solutions. J. Phys. (Paris) Colloq. 38:C7-191-196.

Georgopoulos, P. and Cohen, J. B. 1981. The defect arrangement in (non-stoichiometric) $\beta^{\prime}-\mathrm{NiAl}$ Acta Metall. Mater. 29:1535-1551.

Gragg. J. E., Hayakawa, M., and Cohen, J. B 1973. Errors in quantitative analysis of diffuse scattering from alloys. J. Appl. Crystallogr. 6:59-66.

Hashimoto, S., Iwasaki, H., Ohshima, K., Harada, J., Sakata, M., and Terauchi, H. 1985. Study of local atomic order in a ternary $\mathrm{Cu}_{0.47} \mathrm{~N}_{0.29} \mathrm{Zn}_{0.24}$ alloy using anomalous scattering of synchrotron radiation. J. Phys. Soc. Jpn. 54:3796-3807.

Horiuchi, T., Takizawa, S., Tomoo, S., and Mohri, T. 1995. Computer simulation of local lattice distortion in $\mathrm{Cu}-\mathrm{Au}$ solid solution. Metall. Mater. Trans. A 26A:11-19.

Hoyt, J. J., Fontaine, D. D., and Warburton, W. K 1984. Determination of the anomalous scattering factors for $\mathrm{Cu}, \mathrm{Ni}$ and Ti using the dispersion relation. J. Appl. Crystallogr. 17:344-351.

Ice, G. E. and Sparks, C. J. 1990. Mosaic crystal x-ray spectrometer to resolve inelastic background from anomalous scattering experiments. Nucl. Instrum. Methods A291:110 116.

Ice, G. E., Sparks, C. J., Habenschuss, A., and Shaffer, L. B. 1992. Anomalous X-ray scattering measurement of nearneighbor individual pair displacements and chemical order in $\mathrm{Fe}_{22.5} \mathrm{Ni}_{77.5}$ Phys. Rev. Lett. 68:863-866.

Ice, G. E., Sparks, C. J., Jiang, X., and Robertson, L. 1998. Diffuse scattering measurements of static atomic displacements in crystalline binary solid solutions. J. Phase Equilib. 19:529-537.

Ice, G. E., Sparks, C. J., and Shaffer, L. B. 1994. Chemical and displacement atomic pair correlations in crystalline solid solutions recovered by resonant (anomalous) x-ray scattering. In Resonant Anomalous X-Ray Scattering: Theory and Applications (G. Materlik, C. J. Sparks, and K. Fischer, eds.). pp. 265-294. Elsevier Science Publishing, New York.

James, R.W. 1948. The Optical Principles of the Diffraction of X-Rays. Cornell University Press, Ithaca, N.Y.

Jiang, X., Ice, G. E., Sparks, C. J., Robertson, L., and Zachack, $P$. 1996. Local atomic order and individual pair displacements of $\mathrm{Fe}_{46.5} \mathrm{Ni}_{53.5}$ and $\mathrm{Fe}_{22.5} \mathrm{Ni}_{77.5}$ from diffuse $\mathrm{x}$ ray scattering studies. Phys. Rev. B 54:3211-3226.

Jiang, X., Ice, G. E., Sparks, C. J., and Zschack, P. 1995. Recovery of SRO parameters and pairwise atomic displacements in a $\mathrm{Fe}_{46.5} \mathrm{Ni}_{53.5}$ alloy. In Applications of Synchrotron Radiation Techniques to Materials Science. Mater. Res. Soc. Symp. Proc. 375:267-273.

Kawamura, T. and Fukamachi, T. 1978. Application of the dispersion relation to determine the anomalous scattering factors. Proceedings of the International Conference on X-ray and VUV Spectroscopies, Sendai, Japan. J. Appl. Phys., Suppl. 17-2:224-226.

Kostorz, G. 1996. X-ray and neutron scattering. In Physical Metallurgy, 4th and revised and enhanced edition. (R W. Cahn and P. Hoosen, eds.). pp. 1115-1199. Elsevier Science Publishing, New York.

Larson, B. C. 2009. X-ray Diffuse Scattering Near Bragg Reflections for the Study of Clustered Defects in Crystalline Materials, pp. 139-160 in Diffuse Scattering and the Fundamental Properties of Materials, Momentum Press LLC, New Jersey Ed. R. I. Barabash, G.E. Ice, and P.E. Turchi.

Lengeler, B. 1994. Experimental determination of the dispersion correction $f^{\prime}(E)$ of the atomic scattering factor. In Resonant Anomalous X-Ray Scattering: Theory and Applications (G. Materlik, C. J. Sparks, and K. Fischer, eds.). pp. 35-60. Elsevier Science Publishing, New York.

Miller, M. K., Cerezo, A., Hetherington, M. G., and Smith, G. D. W. 1996. Atom Probe Field Ion Microscopy. Oxford University Press, Oxford.

Mousseau, N. and Thorpe, M. F. 1992. Length distributions in metallic alloys. Phys. Rev. B 45:2015-2022.

Mudgel, M., Awana, V. P. S., Kishan, H. and Bhalla, G. L. 2007 Physica C467:31-37.
Müller, P. P., Schönfeld, B., Kostorz, G., and Bührer, W. 1989. Guinier-Preston I zones in $\mathrm{Al}-1.75$ at. $\% \mathrm{Cu}$ single crystals. Acta Metall. Mater. 37:2125-2132.

Pierron-Bohnes, V., Cadeville, M. C, and Gautier, F. 1983. Magnetism and local order in dilute Fe-C alloys. J. Phys. F 13:1689-1713.

Reinhard, L., Robertson, J. L., Moss, S. C, Ice, G. E., Zschack, P., and Sparks, C. J. 1992. Anomalous-X-ray scattering study of local order in BCC $\mathrm{Fe}_{0.53} \mathrm{Cr}_{0.47}$. Phys. Rev B 45:2662-2676.

Renaud, G., Motta, N., Lançon, F., and Belakhovsky, M. 1988. Topological short-range disorder in $\mathrm{Au}_{1-\mathrm{X}} \mathrm{Ni}_{\mathrm{x}}$ solid solutions: An extended $\mathrm{x}$-ray absorption fine structure spectroscopy and computer-simulation study. Phys. Rev. B 38:5944-5964

Robertson, J. L., Sparks, C. J., Ice, G. E., Jiang, X., Moss, S. C, and Reinhard, L. 1998. Local atomic arrangements in binary solid solutions studied by $x$-ray and neutron diffuse scattering from single crystals. In Local Structure from Diffraction: Fundamental Materials Science Series (M. F. Thorpe and S. Billinge, eds.). Plenum, New York. In press.

Sasaki, S. 1989. Numerical tables of anomalous scattering factors calculated by the Cromer and Liberman's method. KEK Report 88-14.

Scheuer, U. and Lengeler, B. 1991. Lattice distortion of solute atoms in metals studied by $\mathrm{x}$-ray absorption fine structure. Phys. Rev. B 44:9883-9894.

Schönfeld, B., Ice, G. E., Sparks, C. J., Haubold, H.-G., Schweika, W., and Shaffer, L.B. 1994. X-ray study of diffuse scattering in Ni-20 at\% Cr. Phys. Status Solidi B 183:79-95.

Schönfeld, B., Roelofs, H., Kostorz, G. , Robertson, J. L., Zschack, P. and Ice, G. E. 2008. Static Atomic Displacements in $\mathrm{Cu}-\mathrm{Mn}$ Measured with Diffuse X-ray Scattering, Phys. Rev. B. 77144202.

Schwartz, L. H. and Cohen, J. B. 1987. Diffraction from Materials. Springer-Verlag, New York and Heidelberg.

Schweika, W. 1998. Disordered Alloys: Diffuse Scattering and Monte Carlo Simulations, Vol. 141. Springer-Verlag, New York and Heidelberg.

Simon, J. P. and Lyon, O. 1991. The nature of the scattering tail in $\mathrm{Cu}-\mathrm{Ni}-\mathrm{Fe}$ and invar alloys investiaged by anomalous small angle x-ray scattering. J. Appl. Crystallogr. 24:1027-1034.

Sparks, C. J. 1974. Inelastic resonance emission of $x$ rays: Anomalous scattering associated with anomalous dispersion. Phys. Rev. Lett. 33:262-265.

Sparks, C. J. and Borie, B. 1965. Local atomic arrangements studied by x-ray diffraction. In AIME Conference Proceedings 36 (J.B. Cohen and J.E. Hilliard, eds.). pp. 5-50. Gordon and Breach, New York.

Sparks, C. J., Ice, G. E., Shaffer, L. B., and Robertson, J. L. 1994. Experimental measurements of local displacement and chemical pair correlations in crystalline solid solutions. In Metallic Alloys: Experimental and Theoretical Perspectives (J. S. Faulkner and R. G. Jordan, eds.). pp. 73-82. Kluwer Academic Publishers, Dordrecht, The Netherlands, NATO Vol. 256.

Sparks, C. J. and Robertson, J. L. 1994. Guide to some crystallographic symbols and definitions with discussion of short-range correlations. In Resonant Anomalous X-ray Scattering: Theory and Applications (G. Materlik, C. J. Sparks, and K. Fischer, eds.). pp. 653-664. Elsevier Science North-Holland, Amsterdam, The Netherlands.

Suortti, P., Hastings, J. B., and Cox, D. E. 1985. Powder diffraction with synchrotron radiation. I. Absolute measurements. Acta Crystallogr. A41:413-416.

Tibballs, J. E. 1975. The separation of displacement and substitutional disorder scattering: A correction for structure factor ratio variation. J. Appl. Crystallogr. 8:111-114.

Warren, B. E. 1969 (reprinted) 1990. In X-Ray Diffraction. Dover Publications, New York.

Warren, B. E., Averbach, G. L., and Roberts, B. W. 1951. Atomic size effect in the x-ray scattering by alloys. J. Appl. Phys. 22:1493-1496.

Welberry, T. R. and Butler, B. D. 1995. Diffuse x-ray scattering from disordered crystals. Chem Rev. 95:2369-2403.

Wu, T. B., Matsubara, E., and Cohen, J. B. 1983. New procedures for quantitative studies of diffuse x-ray scattering. J. Appl. Crystallogr. 16:407-14.

Zunger, A. 1994. First-principles statistical mechanics of semiconductor alloys and intermetallic compounds. In Statics and Dynamics of Alloy Phase Transitions, Vol. B319 of NATO ASI Series B (P.EA. Turchi and A. Gonis, eds.). p. 361. Plenum, New York. 


\section{KEY REFERENCES}

Cowley, J.M. 1975. Diffraction Physics. North-Holland, Amsterdam, The Netherlands.

This reference is a good basic introduction into diffraction physics and $x$-ray techniques.

Materlik, G., Sparks, C. J., and Fisher, K. (eds.).. 1994. Resonant Anomalous X-ray Scattering. North-Holland, Amsterdam, The Netherlands.

This reference is a collection of recent work on the theory and application of resonant $x$-ray scattering techniques. It provides the most complete introduction to the application of anomalous (resonant) scattering techniques and how the field has been revolutionized by the availability of intense synchrotron radiation sources.

Schwartz and Cohen, 1987. See above.

This is another introductory text on $x$-ray diffraction with an emphasis on the application to materials. The section on diffuse $x$-ray scattering is especially strong and the notation is the same as used in this unit.

Schweika, 1998. See above.

This monograph provides an excellent reference to the interplay between experiment and theory in the field of local atomic order in alloys.

Warren, 1969 (reprinted 1990). See above.

This reference provides an authoritative treatment of all phases of diffuse x-ray scattering, including thermal diffuse scattering, TDS, short-range order, and atomic size displacement scattering. Although it does not include a modern outlook on the importance of resonant scattering, it provides a clear foundation for virtually all modern treatments of diffuse scattering from materials.

\section{APPENDIX A:}

\section{DERIVATION OF THE DIFFUSE INTENSITY}

Our interest is in the diffusely distributed intensity. To separate Equation 3 into an intensity that may be sharply peaked and one that is diffusely distributed, we follow the method of Warren (1969). This method expands $e^{2 \pi i \mathbf{H} \bullet(\delta p-\delta q)}$ in a Taylor series about $\delta_{P}-\delta_{q}$ and examines the displacement terms as the separation of atom pairs becomes large, $p-\mathrm{q} \rightarrow \infty$. As the $\mathrm{x}$-ray or neutron beam intercepts many atoms and the atoms undergo many thermal vibrations during the period of the intensity measurement, both a spatial and a time average are taken. These are indicated by $<>$. The spatial and time average of the $j$ thorder Taylor series expansion of the exponential displacement term is

$$
\begin{aligned}
\left\langle e^{e \pi \mathrm{H}} \bullet\left(\delta_{p}-\delta_{g}\right)\right\rangle \equiv & \left\langle e^{i X_{p q}}\right\rangle=1+i\left\langle X_{p q}\right\rangle-\frac{\left\langle X_{p q}^{2}\right\rangle}{2}-\frac{i\left\langle X_{p q}^{3}\right\rangle}{3 !} \\
& +\ldots+\frac{i^{j}\left\langle X_{p q}^{2}\right\rangle}{j !}
\end{aligned}
$$

The time average for harmonic thermal displacements causes odd-order terms to vanish. With the definition $\left\langle X_{p q}\right\rangle=\left\langle X_{p}-\right.$ $\left.X_{q}\right\rangle$, so that $\left\langle\left(X_{p}-X_{q}\right)^{2}\right\rangle=\left\langle X_{p}^{2}\right\rangle+\left\langle X_{q}^{2}\right\rangle-2\left\langle X_{p} X_{q}\right\rangle$, and for sharply peaked Bragg reflections, where $p-q \rightarrow \infty$, the displacements become uncorrelated so that $\left\langle X_{p} X_{q}\right\rangle=0$. Therefore, $\left\langle X_{p q}^{2}\right\rangle_{p-q \rightarrow \infty}=\left\langle X_{p}^{2}\right\rangle+\left\langle X_{q}^{2}\right\rangle$, and with the harmonic approximation we can estimate the long-range dynamical displacement term by

$$
\left\langle e^{i X_{p q}}\right\rangle_{p-q \rightarrow \infty} \cong e^{\frac{1}{2}\left(\left\langle x_{p}^{2}\right\rangle+\left\langle x_{q}^{2}\right\rangle\right)}=e^{-\left(M_{p}+M_{q}\right)}
$$

Here $M_{p}$ is the usual designation for the Debye-Waller temperature factor (Warren, 1990, p. 35). The subject is treated by Chen in KINEMATIC DIFFRACTION OF X RAYS. We also include the mean-square static displacements in $M$. Experience has shown the validity of the harmonic approximation in Equation 17 to account for the reduction in the intensity of the Bragg reflections as a function of $\mathbf{H}$. With the understanding that when there is an A atom at site $p$ or $q, M_{p}$ or $M_{q}$ is written as $M_{\mathrm{A}}$, and similarly $M_{\mathrm{B}}$ when there is a B at $p$ or $q$, the fundamental Bragg intensity for an alloy is given by the substitution of Equation 17 into Equation 3 as

$$
I(\mathbf{H})_{\text {Fund }}=\left|C_{\mathrm{A}} f_{\mathrm{A}} e^{-M_{A}}\right|+\left.C_{\mathrm{B}} f_{\mathrm{B}} f_{\mathrm{B}} e^{-M_{\mathrm{B}}}\right|^{2} \sum_{p} \sum_{q} e^{2 \pi \mathrm{H} \cdot\left(\mathbf{R}_{p}-\mathbf{R}_{q}\right)}
$$

This expression accounts for the reduced intensity of the fundamental Bragg reflections due to thermal motion and static displacements of the atoms. Fundamental reflections scale as the average scattering factor and are insensitive to how the chemical composition is distributed on the lattice sites. When the alloy has long-range order among the kinds of atoms, the $\alpha_{p q}$ 's do not converge rapidly with larger $p, q$ and account for the superstructure Bragg reflections that depend on how the atoms are distributed among the sites. We are now concerned with the distribution of this thermal and static scattering. To recover the diffuse intensity, we subtract $I(\mathbf{H})_{\text {Fund }}$ from $I(\mathbf{H})_{\text {Total }}$ in Equation 3. To avoid making the harmonic approximation of Equation 17, we subtract $I(\mathbf{H})_{\text {Fund }}$ term by term and take the limit as $p-q \rightarrow \infty$. For example, to second order we have

$$
\left\langle e^{i X_{p q}}\right\rangle_{p-q \rightarrow \infty}=1-\frac{1}{2}\left(\left\langle X_{p}^{2}\right\rangle+\left\langle X_{2}^{q}\right\rangle\right)
$$

By substitution of Equation 19 for each of the $\left\langle e^{i X_{p q}}\right\rangle$ terms in Equation 3 and assignment of the proper atom identity for different pairs, and recalling that as $p-q \rightarrow \infty, \rightarrow \infty, \alpha_{p q}=0$, we subtract this expression for $I(\mathbf{H})_{\text {Fund }}$ from $I(\mathbf{H})_{\text {Total }}$ and write the diffuse scattering to second order in the displacements as

$$
\begin{aligned}
I(\mathbf{H})_{\text {Diffuse }}= & \sum_{p} \sum_{q}\left\{\left(C_{\mathrm{A}}^{2}+C_{\mathrm{A}} C_{\mathrm{B}} \alpha_{p q}\right)\left|f_{\mathrm{A}}\right|^{2}\right. \\
& \times\left(1+i\left\langle X_{p}^{\mathrm{A}}-X_{q}^{\mathrm{A}}\right\rangle-\frac{1}{2}\left\langle\left(X_{p}^{\mathrm{A}}-X_{q}^{\mathrm{A}}\right)^{2}\right\rangle\right) \\
& -C_{\mathrm{A}}^{2}\left|f_{\mathrm{A}}\right|^{2}\left(1-\left\langle X^{2}\right\rangle^{\mathrm{A}}\right)+C_{\mathrm{A}} C_{\mathrm{B}}\left(1-\alpha_{p q}\right) f_{\mathrm{A}} f^{*} \mathrm{~B}^{\mathrm{B}} \\
& \times\left(1+i\left\langle X_{p}^{\mathrm{B}}-X_{p}^{\mathrm{A}}\right\rangle-\frac{1}{2}\left\langle\left(X_{p}^{\mathrm{B}}-X_{p}^{\mathrm{A}}\right)^{2}\right\rangle\right) \\
& -2 C_{\mathrm{A}} C_{\mathrm{B}} f_{\mathrm{A}} f_{\mathrm{B}}^{*}\left(1-\frac{1}{2}\left\langle X^{2}\right\rangle^{\mathrm{A}}-\frac{1}{2}\left\langle X^{2}\right\rangle^{\mathrm{B}}\right) \\
& +C_{\mathrm{A}} C_{\mathrm{B}}\left(1-\alpha_{p q}\right) f_{\mathrm{A}} f_{\mathrm{B}}^{*} \\
& \times\left(1+i\left\langle X_{p}^{\mathrm{A}}-X_{q}^{\mathrm{B}}\right\rangle-\frac{1}{2}\left\langle\left(X_{p}^{\mathrm{A}}-X_{q}^{\mathrm{B}}\right)^{2}\right\rangle\right) \\
& +\left(C_{\mathrm{B}}^{2}+C_{\mathrm{A}} C_{\mathrm{B}} \alpha_{p q}\right)\left|f_{\mathrm{B}}\right|^{2} \\
& \times\left(1+i\left\langle X_{p}^{\mathrm{B}}-X_{q}^{\mathrm{B}}\right\rangle-\frac{1}{2}\left\langle\left(X_{p}^{\mathrm{B}}-X_{q}^{\mathrm{B}}\right)^{2}\right\rangle\right) \\
& \left.-C_{\mathrm{B}}^{2}\left|f_{\mathrm{B}}\right|^{2}\left(1-\left\langle X^{2}\right\rangle^{\mathrm{B}}\right)\right\} e^{2 \pi i \mathrm{H} \bullet(\mathrm{R} p-\mathbf{R} q)}
\end{aligned}
$$

Our use of the double sum requires that the pairs of atoms be counted in both directions, that is, a $p, q$ pair will become a $q, p$ pair such that $i\left(\delta_{p}-\delta_{q}\right)=-i\left(\delta_{q}-\delta_{q}\right)$ as shown in Figure 22. As seen in Equation 2, the $I(\mathbf{H})_{\text {Total }}$ double sum is made up of p, $q$ elements that are the product of four complex numbers: the two complex scattering factors and the two complex phase factors. For every $p, q$ pair there is a corresponding $q, p$ pair where the two scattering factors and the two phase factors are the $p, q$ pair complex conjugates; hence the $p, q$ and $q, p$ elements add up to a real number. This means that the terms in the series expansion of the fluctuation displacements must add in pairs to give real intensity and from Equation 20, the $j=$ odd grder displacement terms have a $\sin 2 \pi(\mathbf{H} \bullet \mathbf{R})\left\langle\left(X_{p}^{\mathrm{A}}-X_{q}^{\mathrm{A}}\right)^{j}\right\rangle$ dependence and the $\left.\left.j_{\mathrm{A}}=\mathrm{even}_{\mathrm{A}}^{\mathrm{A}}\right)^{j}\right\rangle^{\mathrm{A} d e r}$ derms have a cos $2 \pi(\mathbf{H} \cdot \mathbf{R})$
cancel.

From the definition of an average lattice, the weighted average of the displacements for all the kinds of pairs formed for any coordination shell is zero (Warren et al., 1951),

$$
\begin{aligned}
2\left(\alpha_{p q}-1\right)\left\langle\delta_{p}^{\mathrm{A}}-\delta_{q}^{\mathrm{B}}\right\rangle= & \left(\frac{C_{\mathrm{A}}}{C_{\mathrm{B}}}+\alpha_{p q}\right)\left\langle\delta_{p}^{\mathrm{A}}-\delta_{q}^{\mathrm{A}}\right\rangle \\
& +\left(\frac{C_{\mathrm{B}}}{C_{\mathrm{A}}}+\alpha_{p q}\right)\left\langle\delta_{p}^{\mathrm{B}}-\delta_{q}^{\mathrm{B}}\right\rangle
\end{aligned}
$$


If a crystal structure has more than one kind of site symmetry (sublattices with different site symmetries), than Equation 21 may be true for only that sublattice with all the same site symmetries. Different-sized atom species will most likely have a preference for the symmetry of a particular sublattice. This preference could produce long-range correlations and superstructure reflections from which site occupation preferences can be recovered. Disorder among the atoms on any one sublattice and between sublattices will produce scattering from which pair correlations can be recovered. Discussion of this issue is beyond the scope of this unit. A study of a partially ordered non-stoichiometric bcc NiAl crystal, where one of the two sublattices with the same site symmetry is partly occupied by vacancies, has been discussed in Georgopoulos and Cohen (1981). A general review of the application of diffuse scattering measurements to more complicated structures with different site symmetries shows a wider use of models to reduce the number of variables necessary to describe the local pair correlations (Welberry and Butler, 1995).

Equation 20 can be expressed in a more tractable form through three steps: (1) Replace the double sum over atomic sites $p$ and $q$ by $N$ single sums around an origin site where the relative sites are identified by lattice difference $l m n$ such that $\mathbf{R}_{p}$ $-\mathbf{R}_{q}=\mathbf{R}_{0}-\mathbf{R}_{l m n}$. This approximation neglects surface effects. (2) Use trigonometric functions to simplify the phase factors. (3) Substitute Equation 21 into Equation 20. With these steps, the diffuse intensity can be expressed as,

$$
\begin{aligned}
\frac{I(\mathbf{H})_{\text {Diffuse }}}{N}=\sum_{l m n} & C_{\mathrm{A}} C_{\mathrm{B}}\left|f_{\mathrm{A}}-f_{\mathrm{B}}\right|^{2} \alpha_{l m n} \cos 2 \pi \mathbf{H} \bullet\left(\mathbf{R}_{0}-\mathbf{R}_{l m n}\right) \\
& -\sum_{l m n}\left[\left(C_{\mathrm{A}}^{2}+C_{\mathrm{A}} C_{\mathrm{B}} \alpha_{l m n}\right) \operatorname{Re}\left(f_{\mathrm{a}}\left(f_{\mathrm{A}}-f_{\mathrm{B}}\right)^{*}\right)\right. \\
& \times\left\langle X_{0}^{\mathrm{A}}-X_{l m n}^{\mathrm{A}}\right\rangle-\left(C_{\mathrm{B}}^{2}+C_{\mathrm{A}} C_{\mathrm{B}} \alpha_{l m n}\right) \operatorname{Re}\left(f_{\mathrm{B}}\left(f_{\mathrm{A}}-f_{\mathrm{B}}\right)^{*}\right) \\
& \left.\times\left\langle X_{0}^{\mathrm{B}}-X_{l m n}^{\mathrm{A}}\right\rangle\right] \sin 2 \pi \mathbf{H} \bullet\left(\mathbf{R}_{0}-\mathbf{R}_{l m n}\right) \\
& +\sum_{l m n}\left[C _ { \mathrm { A } } ^ { 2 } | f _ { \mathrm { A } } | ^ { 2 } \left(\left\langle X^{2}\right\rangle^{\mathrm{A}}-\left(1+\frac{C_{\mathrm{B}}}{C_{\mathrm{A}}} \alpha_{l m n}\right)\right.\right. \\
& \left.\times \frac{1}{2}\left\langle\left(X_{0}^{\mathrm{A}}-X_{l m n}^{\mathrm{A}}\right)^{2}\right\rangle\right)+C_{\mathrm{A}} C_{\mathrm{B}} f_{\mathrm{A}} f_{\mathrm{B}}^{*}\left(\left\langle X^{2}\right\rangle^{\mathrm{A}}+\left\langle X^{2}\right\rangle^{\mathrm{B}}\right. \\
& \left.\times\left(1-\alpha_{l m n}\right)\left\langle\left(X_{0}^{\mathrm{B}}-X_{l m n}^{\mathrm{A}}\right)^{2}\right\rangle\right)+C_{\mathrm{B}}^{2}\left|f_{\mathrm{B}}\right|^{2}\left(\left\langle X^{2}\right\rangle^{\mathrm{B}}\right. \\
& \left.\left.-\left(1+\frac{C_{\mathrm{A}}}{C_{\mathrm{B}}} \alpha_{l m n}\right) \frac{1}{2}\left\langle\left(X_{0}^{\mathrm{B}}-X_{l m n}^{\mathrm{B}}\right)^{2}\right\rangle\right)\right] \\
& \times \cos 2 \pi \mathrm{H} \bullet\left(\mathbf{R}_{0}-\mathbf{R}_{l m n}\right)
\end{aligned}
$$

Equation 22 is a completely general description of the diffuse scattering from any crystal structure through the second moment of the static and thermal displacements. We now apply this to binary solid solutions, which have received the most attention among crystalline solid solutions.

It is helpful to choose real-space basis vectors $\mathbf{a}, \mathbf{b}, \mathbf{c}$ that reflect the long-range periodicity of the crystal structure. This long-range periodicity in turn is reflected in the intensity distribution in the reciprocal space lattice with basis vectors $\mathbf{a}^{*}=$ $1 / \mathbf{a}, \mathbf{b}^{*}=1 / \mathbf{b}$, and $\mathbf{c}^{*}=1 / \mathbf{c}$, as shown in Figure 3 . For an alloy that is on average statistically cubic, such as that shown in Figure 3, we define

$$
\mathbf{R}_{0}-\mathbf{R}_{l m n} \equiv \frac{l}{2} \mathbf{a}+\frac{m}{2} \mathbf{b}+\frac{n}{2} \mathbf{c}, \mathbf{H} \equiv h_{1} \mathbf{a}^{*}+h_{2} \mathbf{b}^{*}+h_{3} \mathbf{c}^{*}
$$

so that

$$
2 \pi \mathbf{H} \bullet\left(\mathbf{R}_{0}-\mathbf{R}_{l m n}\right)=\pi\left(h_{1} l+h_{2} m+h_{3} n\right)
$$

In addition,

$$
\delta_{l m n} \equiv \Delta X_{l m n} \mathbf{a}+\Delta Y_{l m n} \mathbf{b}+\Delta Z_{l m n} \mathbf{c}
$$

So that

$$
\begin{aligned}
X_{0}-X_{l m n} \equiv & 2 \pi \mathbf{H} \bullet\left(\delta_{0}-\delta_{l m n}\right) \equiv 2 \pi\left[h_{1}\left(\Delta X_{0}-\Delta X_{l m n}\right)+h_{2}\right. \\
& \left.+\left(\Delta Y_{0}-\Delta Y_{l m n}\right)+h_{3}\left(\Delta Z_{0}-\Delta Z_{l m n}\right)\right]
\end{aligned}
$$

This definition of $\mathbf{R}_{0}-\mathbf{R}_{l m n}$ causes the continuous variables $h_{1} h_{2} h_{3}$ in reciprocal space to have the integer values of the Miller indices at reciprocal lattice points. We further specify that the site symmetry is cubic such as for the bcc Fe structure and fcc $\mathrm{Cu}$ structure. With these definitions, the various diffuse $\mathrm{x}$-ray scattering terms in Equation 22 can be written, starting with the local chemical order term as

$$
\frac{I(\mathbf{H})_{\text {SRO }}}{N}=\sum_{l m n} C_{\mathrm{A}} C_{\mathrm{B}}\left|f_{\mathrm{A}}-f_{\mathrm{B}}\right|^{2} \alpha_{l m n} \cos \pi\left(\mathrm{h}_{1} l+h_{2} m+h_{3} n\right)
$$

which was first given by Cowley (1950). For bcc Fe and fcc $\mathrm{Cu}$ structures,

$\alpha_{l m n}=\alpha_{\overline{l m n}}=\alpha_{\overline{l m} n}=\alpha_{l m \bar{n}}=\alpha_{l \bar{m} n}=\alpha_{l \overline{l m n}}=\alpha_{\overline{l m} n}=\alpha_{\overline{l m} \bar{n}}$
and the cosine term takes the form $\cos \pi h_{1} l \cos \pi h_{2} m \cos \pi h_{3} n$. and the cosine term takes the form $\cos \pi h$
For the first-order displacement term,

$$
\begin{aligned}
& \frac{I(\mathbf{H})_{j=1}}{N}=-\operatorname{Re}\left(f_{\mathrm{A}}\left(f_{\mathrm{A}}^{*}-f_{\mathrm{B}}^{*}\right)\right)\left[h_{1} Q_{X}^{\mathrm{AA}}+h_{2} Q_{Y}^{\mathrm{AA}}+h_{3} Q_{Z}^{\mathrm{AA}}\right] \\
& +\operatorname{Re}\left(f_{\mathrm{B}}\left(f_{\mathrm{A}}^{*}-f_{\mathrm{B}}^{*}\right)\right)\left[h_{1} Q_{X}^{\mathrm{BB}}+h_{2} Q_{Y}^{\mathrm{BB}}+h_{3}^{\mathrm{BB}}\right]
\end{aligned}
$$

Where

$$
\begin{aligned}
Q_{X}^{\mathrm{AA}}=2 & \pi \sum_{l m n}\left(C_{\mathrm{A}}^{2}+C_{\mathrm{A}} C_{\mathrm{B}} \alpha_{l m n}\right)\left\langle\Delta X_{l m n}^{\mathrm{A}}\right\rangle_{0}^{\mathrm{A}} \sin \pi h_{1} l \\
& \times \cos \pi h_{2} m \cos \pi h_{3} n
\end{aligned}
$$

and

$$
\begin{aligned}
Q_{X}^{\mathrm{BB}} & =2 \pi \sum_{l m n}\left(C_{\mathrm{B}}^{2}+C_{\mathrm{A}} C_{\mathrm{B}} \alpha_{l m n}\right)\left\langle\Delta X_{l m n}^{\mathrm{B}}\right\rangle_{0}^{\mathrm{B}} \sin \pi h_{1} l \\
& \times \cos \pi h_{2} m \cos \pi h_{3} n
\end{aligned}
$$

and similarly for the other terms as given by Borie and Sparks (1964) and Georgopoulos and Cohen (1977). Where

$$
\left\langle\Delta X_{l m n}^{\mathrm{A}}\right\rangle_{0}^{\mathrm{A}}=\left\langle\Delta X_{0}^{\mathrm{A}}-\Delta X_{l m n}^{\mathrm{A}}\right\rangle
$$

Equation 28 is a result given by Borie and Sparks (1964) that avoids an earlier assumption of radial displacements first given by Warren et al. (1951). Shown in Figure 23 is a schematic of the displacements described by Equation 28.

Diffuse scattering from the second-order displacement term can be expanded as,

$$
\begin{aligned}
\frac{I(\mathbf{H})_{j=2}}{N}= & \left|f_{\mathrm{A}}\right|^{2}\left(h_{1}^{2} R_{X}^{\mathrm{AA}}+h_{2}^{2} R_{Y}^{\mathrm{AA}}+h_{3}^{2} R_{Z}^{\mathrm{AA}}\right) \\
& +f_{\mathrm{A}} f_{\mathrm{B}}\left(h_{1}^{2} R_{X}^{\mathrm{AB}}+h_{2}^{2} R_{Y}^{\mathrm{AB}}+h_{3}^{2} R_{Z}^{\mathrm{AB}}\right) \\
& +\left|f_{\mathrm{B}}\right|^{2}\left(h_{1}^{2} R_{X}^{\mathrm{BB}}+h_{2}^{2} R_{Y}^{\mathrm{BB}}+h_{3}^{2} R_{Z}^{\mathrm{BB}}\right) \\
& +\left|f_{\mathrm{A}}\right|^{2}\left(h_{1} h_{2} S_{X Y}^{\mathrm{AA}}+h_{1} h_{3} S_{X Z}^{\mathrm{AA}}+h_{2} h_{3} S_{Y Z}^{\mathrm{AA}}\right) \\
& +f_{\mathrm{A}} f_{\mathrm{B}}^{*}\left(h_{1} h_{2} S_{X Y}^{\mathrm{AB}}+h_{1} h_{3} S_{X Z}^{\mathrm{AB}}+h_{2} h_{3} S_{Y Z}^{\mathrm{AB}}\right) \\
& +\left|f_{\mathrm{B}}\right|^{2}\left(h_{1} h_{2} S_{X Y}^{\mathrm{BB}}+h_{1} h_{3} S_{X Z}^{\mathrm{BB}}+h_{2} h_{3} S_{Y Z}^{\mathrm{BB}}\right)
\end{aligned}
$$

Where

$$
\begin{aligned}
R_{X}^{\mathrm{AA}}= & 4 \pi^{2} C_{\mathrm{A}}^{2} \sum_{l m n}\left\{\left\langle\Delta X^{2}\right\rangle^{\mathrm{A}}-\left(1+\frac{C_{\mathrm{B}}}{C_{\mathrm{A}}} \alpha_{l m n}\right)\right. \\
& \left.\times\left[\left\langle\left(\Delta X^{2}\right)_{l m n}^{\mathrm{A}}\right\rangle_{0}^{\mathrm{A}}-\left\langle\Delta X_{0}^{\mathrm{A}} \Delta X_{l m n}^{\mathrm{A}}\right\rangle\right]\right\} \\
& \times \cos \pi\left(h_{1} l+h_{2} m=h_{3} n\right)
\end{aligned}
$$

and similarly for $R_{Y}$ and $R_{Z}$ with $Y$ and $Z$ replacing $X$, respectively, or $R_{X}^{\mathrm{AA}}\left(h_{1} h_{2} h_{3}\right)=R_{Y}^{\mathrm{AA}}\left(h_{2} h_{3} h_{1}\right)$. The $R^{B B}$ terms are given by

$$
\begin{aligned}
R_{X}^{\mathrm{BB}}= & 4 \pi^{2} C_{\mathrm{B}}^{2} \sum_{l m n}\left\{\left\langle\Delta X^{2}\right\rangle^{\mathrm{B}}-\left(1+\frac{C_{\mathrm{A}}}{C_{\mathrm{B}}} \alpha_{l m n}\right)\right. \\
& \left.\times\left[\left\langle\left(\Delta X^{2}\right)_{l m n}^{\mathrm{B}}\right\rangle_{0}^{\mathrm{B}}-\left\langle\Delta X_{0}^{\mathrm{B}} \Delta X_{l m n}^{\mathrm{B}}\right\rangle\right]\right\} \\
& \times \cos \pi\left(h_{1} l+h_{2} m=h_{3} n\right)
\end{aligned}
$$

For the $R^{\mathrm{AB}}$ terms, we have

$$
\begin{aligned}
R_{X Y}^{\mathrm{AB}}= & 4 \pi^{2} C_{\mathrm{A}} C_{\mathrm{B}} \sum_{l m n}\left[\left\langle\Delta X^{2}\right\rangle^{\mathrm{A}}+\left\langle\Delta X^{2}\right\rangle^{\mathrm{B}}-\left(1-\alpha_{l m n}\right)\right. \\
& \left.\times\left(\left\langle\left(\Delta X^{2}\right)_{l m n}^{\mathrm{A}}\right\rangle_{0}^{\mathrm{B}}+\left\langle\left(\Delta X^{2}\right)_{0}^{\mathrm{B}}\right\rangle_{l m n}^{\mathrm{A}}-2\left\langle\Delta X_{0}^{\mathrm{B}} \Delta X_{l m n}^{\mathrm{A}}\right\rangle\right)\right] \\
& \times \cos \pi\left(h_{1} l+h_{2} m=h_{3} n\right)
\end{aligned}
$$

and similar terms for $R_{Y}^{\mathrm{AB}}$ with $Y$ replacing $X$ and for $R_{Z}^{\mathrm{AB}}$ with $Z$ replacing $X$. For the cross terms 


$$
\begin{aligned}
S_{X Y}^{\mathrm{AA}}= & 4 \pi^{2} C_{\mathrm{A}}^{2} \sum_{l m n}\left(1+\frac{C_{\mathrm{B}}}{C_{\mathrm{A}}} \alpha_{l m n}\right)\left\langle\Delta X_{0}^{\mathrm{A}}-\Delta X_{l m n}^{\mathrm{A}}\right\rangle \\
& \times\left\langle\Delta Y_{0}^{\mathrm{A}}-\Delta Y_{l m n}^{\mathrm{A}}\right\rangle \cos \pi\left(h_{1} l+h_{2} m+h_{3} n\right)
\end{aligned}
$$

and similarly for $S_{X Z}^{\mathrm{AA}}$ and $S_{Y Z}^{\mathrm{AA}}$ with $X Y$ replaced with $X Z$
and $Y Z$, respectively. The terms $S_{X Y}^{\mathrm{BB}}, S_{X Z}^{\mathrm{BB}}$ and $S_{Y Z}^{\mathrm{BB}}$ are derived from the $S^{\mathrm{AA}}$ terms by replacing $\mathrm{AA}$ with $\mathrm{BB}$ and by replacing $C_{\mathrm{A}}^{2}$, with $C_{\mathrm{B}}^{2}$ and $C_{\mathrm{A}} / C_{\mathrm{B}}$ with $C_{\mathrm{B}} / C_{\mathrm{A}}$. For the term $S_{X Y}^{\mathrm{AB}}$, we write

$$
\begin{aligned}
S_{X Y}^{\mathrm{AB}}= & 8 \pi^{2} C_{\mathrm{A}} C_{\mathrm{B}} \sum_{l m n}\left(1-\alpha_{l m m}\right)\left\langle\left(\Delta X_{0}^{\mathrm{B}}-\Delta X_{l m n}^{\mathrm{A}}\right)\right. \\
& \left.\times\left(\Delta Y_{0}^{\mathrm{B}}-\Delta Y_{l m n}^{\mathrm{A}}\right)\right\rangle \cos \pi\left(h_{1} l+h_{2} m=h_{3} n\right)
\end{aligned}
$$

and similarly for $S_{X Z}^{\mathrm{AB}}$ and $S_{Y Z}^{\mathrm{AB}}$, where $X Z$ and $Y Z$ replace $X Y$, respectively. The periodicity of the terms in Equations 27, 28, and 29 , and the assumption that the scattering factor terms can be made independent of $\mathbf{H}$, permit us to write their sum as

$$
\begin{aligned}
\frac{I(\mathbf{H})_{\text {Diffuse }}}{N \mid f\left(\left.\mathbf{H}\right|^{2}\right)}= & \mathrm{A}\left(h_{1} h_{2} h_{3}\right)+h_{1} \mathrm{~B}\left(h_{1} h_{2} h_{3}\right)+h_{2} \mathrm{~B}\left(h_{2} h_{3} h_{1}\right) \\
& +h_{3} \mathrm{~B}\left(h_{3} h_{1} h_{2}\right)+h_{1}^{2} \mathrm{C}\left(h_{1} h_{2} h_{3}\right)+h_{2}^{2} \mathrm{C}\left(h_{2} h_{3} h_{1}\right) \\
& +h_{3}^{2} \mathrm{C}\left(h_{3} h_{1} h_{2}\right)+h_{1} h_{2} \mathrm{D}\left(h_{1} h_{2} h_{3}\right) \\
& +h_{1} h_{3} \mathrm{D}\left(h_{2} h_{3} h_{1}\right)+h_{2} h_{3} \mathrm{D}\left(h_{3} h_{1} h_{2}\right)
\end{aligned}
$$

where $\mathrm{A}\left(h_{1}, h_{2}, h_{3}\right)$ is given by Equation $277_{\mathrm{A}} \div|f(\mathbf{H})|^{2}, Q_{X}^{\mathrm{Bgfl}}$ given by Equation $28 \div|f(\mathbf{H})|^{2}$, and likewise for the other terms.

\section{APPENDIX B: \\ NEUTRON MAGNETIC DIFFUSE SCATTERING}

The elastic diffuse scattering of neutrons from binary alloys with magnetic short-range order is composed of three parts: the nuclear scattering, the magnetic scattering, and the nuclear magnetic interference scattering. The nuclear scattering length for neutrons is analogous to the x-ray atomic scattering factor for $\mathrm{x}$ rays. Thus the information obtained is the same for chemical short-range order and displacements as discussed for $\mathrm{x}$ rays. Because neutrons have a magnetic moment, there is also magnetic scattering associated with the unpaired electron spins (see Magnetic Neutron SCATtering). If the direction of magnetization is perpendicular to the scattering plane, the magnetic scattering cross-section (in barns) for an A-B alloy of atomic concentration $C_{\mathrm{A}}$ of $\mathrm{A}$ atoms is given by

$$
I_{\mathrm{M}}(\mathbf{H})=C_{\mathrm{A}} C_{\mathrm{B}} T(\mathbf{H})(0.270)^{2}
$$

Here, $T(\mathbf{H})$ is the moment-moment correlation function expressed in terms of $\mu_{l m n} f_{l m n}(\mathbf{H})$, where $\mu_{l m n}$ is the magnetic moment of the atom on site $l m n$ and $f_{l m n}(\mathbf{H})$ its magnetic form factor (the magnetic scattering comes from the unpaired electrons rather than the nucleus so that it has a scattering angle dependent form factor much like that for $\mathrm{x}$ rays):

$$
\begin{gathered}
\mu_{l m n}=\mu_{l m n} f_{l m n}(\mathbf{H}) \\
C_{\mathrm{A}} C_{\mathrm{B}} T(\mathbf{H})=\sum_{l m n} e_{l m n}^{2 \pi \mathrm{H} \cdot \mathrm{R}}\left\langle\mu_{l m m}(\mathbf{H})\left[\mu_{000}(\mathbf{H})-\langle\mu(\mathbf{H})\rangle\right]\right\rangle
\end{gathered}
$$

The nuclear magnetic interference term $I_{\mathrm{NM}}(\mathbf{H})$ is proportional to a site occupation-magnetic moment correlation $M(\mathbf{H})$. For a magnetization perpendicular to the scattering plane, we have

$$
I_{\mathrm{NM}}(\mathbf{H})=C_{\mathrm{A}} C_{\mathrm{B}} \Delta b(0.540) M(\mathbf{H})
$$

and

$$
C_{\mathrm{A}} C_{\mathrm{B}} M(\mathbf{H})=\sum_{l m n} e_{l m n}^{2 \pi \mathrm{H} \cdot \mathbf{R}}\left\langle\left(\alpha_{l m n}-1\right) \mu_{000}(\mathbf{H})\right\rangle
$$

Here the quantity in <>'s represents the average increase in the moment of the atom at the origin $(000)$ due to the atomic species of the atom located at site $l m n$.

While the terms $I_{\mathrm{M}}(\mathbf{H})$ and $I_{\mathrm{NM}}(\mathbf{H})$ allow one to study the magnetic short-range order in the alloy, they also complicate the data analysis by making it difficult to separate these two terms from the chemical SRO. One experimental method for resolving the magnetic components is to use polarization analysis where the moment of the incident neutron beam is polarized to be either parallel ( $\varepsilon=1$ ) or antiparallel $(\varepsilon=-1)$ to the magnetization. The total scattering for each case can now be written as

$$
I_{\varepsilon}(\mathbf{H})=I_{\mathrm{N}}(\mathbf{H})+\varepsilon I_{\mathrm{NM}}(\mathbf{H})+I_{\mathrm{M}}(\mathbf{H})
$$

The intensity difference between the two polarization states gives

$$
\Delta I_{\text {Total }}(\mathbf{H})=2 I_{\mathrm{NM}}(\mathbf{H})
$$

and the sum gives

$$
\sum_{\varepsilon} I_{\varepsilon}(\mathbf{H})+2 I_{\mathrm{N}}(\mathbf{H})+2 I_{\mathrm{M}}(\mathbf{H})
$$

If $I_{\mathrm{N}}(\mathbf{H})$ is known from a separate measurement with x rays, all three components of the scattering can be separated from one another.

One of the greatest difficulties in studying magnetic shortrange order comes when the moments of the atoms cannot be aligned in the same direction with, for example, an external magnetic field. In the above analysis, it was assumed that the moments are perpendicular to the scattering vector, $\mathbf{H}$. The magnetic scattering cross-section is reduced by the sine of the angle between the magnetic moment and the scattering vector. Thus if the magnetization is not perpendicular to the scattering vector, the moments on the atoms must be reduced by the appropriate amount. When the spins are not aligned, the sine of the angle between the moment and the scattering vector for each individual atom must be considered. In this case, it becomes necessary to construct computer models of the spin structure to extract $M(\mathbf{H})$ and $T(\mathbf{H})$. More in-depth discussion is given in MAGNETIC NEUTRON SCATTERING.

\section{GENE E. ICE \\ JAMES L. ROBERTSON \\ CUlLIE J. SPARKS \\ Oak Ridge National Laboratory \\ Oak Ridge, Tennessee}

\section{Figure Caption:}

Figure 1. Direct and reciprocal space representations for a clustering, a random, and an ordering $\mathrm{A}_{50} \mathrm{~B}_{50}$ bcc alloy. Courtesy of Robertson et al. (1998).

Figure 2. Displacements about the average lattice preserve the regular spacing between atomic planes such that $d=d_{1}=d_{2}=$ $d_{3}=\ldots$. The average lattice is obtained from the positions of the sharp Bragg reflections (B). Information about short-range correlations among the atoms is contained in the diffusely distributed intensity between the Bragg peaks. Courtesy of Ice et al. (1998).

Figure 3. The atom positions for a face-centered cubic (fcc) structure are used to illustrate the notation for the real-space lattice. The unit cell has dimensions $\mathbf{a}=\mathbf{b}=\mathbf{c}$. The corresponding reciprocal space lattice is $\mathbf{a}^{*}, \mathbf{b}^{*}, \mathbf{c}^{*}$. A position in reciprocal space at which the scattered intensity, $I(\mathbf{H})$, is measured for an incoming $\mathrm{x}$ ray in the direction of $\mathbf{S}_{0}$ of wavelength $\lambda$ and detected in the outgoing direction of $\mathbf{S}$ would be $\mathbf{H}=\left(\mathbf{S}-\mathbf{S}_{0}\right) / \lambda=h_{1} \mathbf{a}^{*}+h_{2} \mathbf{b}^{*}+h_{3} \mathbf{c}^{*}$. At Bragg reflections, $h_{1} h_{2} h_{3}$ are integers and are usually designated $h k l$, the Miller indices of the reflection. This notation follows that used in the International Tables for Crystallography. Courtesy of Sparks and Robertson (1994).

Figure 4. Variation in the ratio of the $\mathrm{x}$-ray atomic scattering factor terms as a function $\mathbf{H}$. The divisor $\langle f\rangle^{2}=\mid C_{\mathrm{Cu}} f_{\mathrm{Cu}}+C_{\mathrm{Au}} f_{\mathrm{Au}}$ $1^{2}$ was chosen to reduce the $\mathbf{H}$ dependence of all the terms for an incident energy of Mo $K_{\alpha}=1.748 \mathrm{keV}$. The relatively larger xray atomic scattering factor of $\mathrm{Au}, f_{\mathrm{Au}}=79$ versus $\mathrm{Cu}, f_{\mathrm{Cu}}=29$ at $\mathbf{H}=0$, would require a divisor more heavily weighted with $f_{A w}$ such as $\left|f_{\mathrm{Au}}\right|^{2}$ to reduce the $\mathbf{H}$ dependence of those terms.

Figure 5. For elements nearby in the periodic table, x-ray energies can be chosen to obtain near null Laue scattering to separate intensity arising from quadratic and higher moments in atomic displacements. Courtesy of Reinhard et al. (1992).

Figure 6. Diffusely scattered $\mathrm{x}$-ray intensity from an $\mathrm{Fe}_{63 \cdot 2} \mathrm{Ni}_{36.8}$ Invar alloy associated with the chemical order $I_{\mathrm{SRO}}$ and the first moment of the static displacements $I_{J=I}$ versus $h$ in reciprocal lattice units (r.l.u.) along the $\left[h_{1} 00\right]$ direction. A major intensity change is affected by the choice of two different $\mathrm{x}$-ray energies. The solid lines calculated from the $\alpha$ and $\delta$ parameters recovered from the $3 \lambda$ data sets closely fit the observed data given by o and 
+. The dashed lines are calculated intensity through the fundamental reflections. Courtesy of Ice et al. (1998).

Figure 7. The diffuse intensity is mapped in a volume of reciprocal space bounded by three mirror planes that contain all the information available for a cubic alloy.

Figure 8. Optical setup for resonant diffuse x-ray scattering measurement.

Figure 9. Variation of $f^{\prime}$ and $f^{\prime \prime}$ near the $K$ absorption edge of nickel. Dashed lines are the theoretical calculation from Cromer and Liberman (1970; 1981). Courtesy of Ice et al. (1994).

Figure 10. Energy spectrum measured with a solid-state detector from Ni-Fe alloy excited by $8.0-\mathrm{keV}$ x rays. Courtesy of Ice et al. (1994)

Figure 11. (A) Scattered radiation is energy analyzed with a mosaic graphite crystal dispersing radiation along a position sensitive detector to resolve (B) fluorescence and (C) resonant Raman and Compton scattering. Courtesy of Ice and Sparks (1990).

Figure 12. Incident beam spread on the sample depends on $\chi$ and $\theta$, which orient the surface normal with respect to the incident beam. As the beam spread on the sample is proportional to the effective source size viewed by the spectrometer, the energy resolution changes as $\chi$ and $\theta$ change. The measured energy resolution (points) is plotted for $\chi=55^{\circ}$ and compared with the theoretical prediction (line).

Figure 13. Energy spectrum of scattered radiation when the incident energy is (A) $13 \mathrm{eV}$ below the Ni $K$ edge and (B) $20 \mathrm{eV}$ below the Fe $K$ edge of a Fe-Ni crystal. Courtesy of Ice et al. (1994).

Figure 14. The usual tabulated values of the resonant (anomalous) scattering terms are not corrected for hole width (lifetime), which causes a Lorentzian broadening of the absorption edge and affects the values of $f^{\prime}$ and $f^{\prime \prime}$ near the edge. Courtesy of Ice et al. (1994).

Figure 15. The absorption edge energy shifts are very small for metallic alloys with differing nearest neighbors. Courtesy of Ice et al. (1994).

Figure 16. Fitted $I_{\mathrm{SRO}}$ along the $h 00$ line for three relative scale factors on the near zero contrast data of a $\mathrm{Ni}_{77.5} \mathrm{Fe}_{22.5}$ sample. With a scale factor of $1.04, I_{\mathrm{SRO}}$ is near zero at the origin and fundamental Bragg peaks as measured by SAXS. Courtesy of Sparks et al (1994).

Figure 17. Total elastically scattered $x$-ray intensity along the (h00) measured at $293 K$ for the three x-ray energies listed. Note the shift in contrast for intensities measured with energies $20 \mathrm{eV}$ below the $\mathrm{Fe} K$ edge at $7092-\mathrm{eV}$ and the Ni $K$ edge at $8313 \mathrm{eV}$, which changes the sign of $\operatorname{Re}\left(f_{\mathrm{Ni}}-f_{\mathrm{Fe}}\right)$. The outlying data point at the $\{100\}$ position is from harmonic energy contamination of the incident radiation and such points are removed before processing. Courtesy of Jiang et al. (1996).

Figure 18. Diffuse x-ray scattering intensities from $\mathrm{Fe}_{22.5} \mathrm{Ni}_{72.5}$ in the $h_{3}=0$ plane collected with x-ray energies of (A) 7.092, (B) 8.000, and (C) $8.313 \mathrm{keV}$. Courtesy of Ice et al. (1992).

Figure 19. Construction of the vectors recovered from diffuse scattering measurements on single crystals. The parameter $\mathbf{R}_{l m n}$ is obtained from the lattice parameter $|\mathbf{a}|$, and the average components of the displacement $\delta_{l m n}^{i j}$ are recovered from measurements of the diffuse scattering. Courtesy of Ice et al. (1998).

Figure 20. Radial displacements (parallel to the interatomic vector $\mathbf{R}_{l m n}$ ) between the atom pairs require that the relative magnitudes of the displacement components be in the same proportion as the average lattice vector; $\Delta X: \Delta \mathrm{Y}: \Delta Z=l, m, n$. As shown for $l m n=211$, a radial displacement requires $|\Delta X|=$ $2|\Delta Y|$ and $|\Delta X|=2|\Delta Z|$. For $l m n=121,|\Delta X|=|\Delta Y| / 2$ and $|\Delta X|=$ $|\Delta Z|$. For a cubic lattice, we can interchange $l, m$, and $n$ and similarly $\Delta X, \Delta Y$, and $\Delta Z$. Thus there is only one value $\Delta X$ for Imn multiplicities $<24$ (i.e., 110, 200, and 222), two values for $\Delta X$ when $l m n$ has multiplicities equal to $24(l \neq m$ and $l=m$, $n)$, and three values for $\Delta X$ with multiplicities equal to 48 . Courtesy of Ice et al. (1998).

Figure 21. Displacement from the average lattice sites for chemically specific pairs. Shell radius divided by the lattice parameter $\boldsymbol{a}_{0}$ becomes 1 for second neighbors (seperated by the cube edge). Courtesy of Ice et al. (1998).
Figure 22. Schematic illustration showing that since all the pairs of atoms are counted as to kind and displacement in both directions, odd-power terms in the displacements are replaced with their negatives $i\left(\delta_{p}-\delta_{q}\right)=-i\left(\delta_{q}-\delta_{q}\right)$ and $\mathbf{R}_{p}-\mathbf{R}_{q}=-\left(\mathbf{R}_{q}\right.$ $-\mathbf{R}_{p}$ ) so that the imaginary terms cancel.

Figure 23. The rectangular square of solid lines is the average lattice about which the atom centers $(+)$ are displaced by the amount $\delta_{p q}$. Shown in the smaller box on the right are the rectangular components of the displacement, $\Delta x, \Delta y$, and $\Delta z$. Courtesy of Jiang et al. (1996). 
Table 1. Contributions to the Uncertainties in the Short-Range-Order Parameter, of $\mathbf{F e}_{46.5} \mathbf{N i}_{53.5}{ }^{a}( \pm \mathbf{l} \boldsymbol{\sigma})$

\begin{tabular}{|c|c|c|c|c|c|c|c|}
\hline $\operatorname{lm} n$ & $\alpha_{\operatorname{lmn}}\left(\sigma_{\text {Total }}\right)$ & $\sigma(\sqrt{n})$ & $\sigma\left(f^{\prime}\right) \pm 0.2 \mathrm{eu}$ & $\sigma\left(P_{0}\right) \pm 1 \%$ & $\sigma(\mathrm{RRS}) \pm 1 \mathrm{eu}$ & $\sigma_{\text {Compton }}$ & $\sigma\left(C_{\mathrm{A}}\right) \pm 0.3$ at. $\%$ \\
\hline 000 & $1.0000(100)$ & 0.0024 & 0 & 0 & 0 & 0 & 0 \\
\hline 110 & $-0.0766(54)$ & 0.0018 & 0.0010 & $\begin{array}{l}0.004 \\
8\end{array}$ & 0 & 0.0006 & 0.0011 \\
\hline 200 & $0.0646(28)$ & 0.0017 & 0.0003 & $\begin{array}{l}0.001 \\
6\end{array}$ & 0.0008 & 0.0013 & 0.0003 \\
\hline 211 & $-0.0022(15)$ & 0.0014 & 0 & $\begin{array}{l}0.000 \\
4\end{array}$ & 0.0001 & 0.0002 & 0.0001 \\
\hline 220 & $0.0037(14)$ & 0.0013 & 0.0002 & $\begin{array}{l}0.000 \\
3\end{array}$ & 0.0003 & 0.0003 & 0.0001 \\
\hline 310 & $-0.0100(11)$ & 0.0011 & 0.0001 & $\begin{array}{l}0.000 \\
2\end{array}$ & 0.0001 & 0.0001 & 0.0001 \\
\hline 222 & $0.0037(12)$ & 0.0011 & 0 & $\begin{array}{l}0.000 \\
2\end{array}$ & 0.0002 & 0.0003 & 0 \\
\hline 321 & $0.0032(19)$ & 0.0009 & 0 & $\begin{array}{l}0.000 \\
1\end{array}$ & 0.0001 & 0.0001 & 0.0001 \\
\hline 400 & $0.0071(12)$ & 0.0011 & 0.0002 & $\begin{array}{l}0.000 \\
1\end{array}$ & 0.0003 & 0.0004 & 0 \\
\hline 330 & $-0.0021(9)$ & 0.0008 & 0.0001 & 0 & 0.0003 & 0.0001 & 0 \\
\hline 411 & $0.0007(7)$ & 0.0007 & 0 & 0 & 0 & 0.0002 & 0 \\
\hline 420 & $0.0012(8)$ & 0.0007 & 1.0002 & 0 & 0.0004 & 0.0001 & 0 \\
\hline 332 & $-0.0007(7)$ & 0.0007 & 0 & 0 & 0 & 0.0001 & 0 \\
\hline
\end{tabular}

${ }^{a}$ For statistical and possible systematic errors associated with counting statistics $n$, the real part of the resonant x-ray scattering factor $f^{\prime}$ the scaling parameter $P_{0}$ to absolute intensities, inelastic resonant Raman scattering (RRS) and Compton contributions, and concentration $C_{\mathrm{A}}$. Total error is shown in parentheses and 0 indicates uncertainties $<0.00005 \AA$.

Table 2. Standard Deviation of $\pm \mathbf{l} \sigma$ of $x, y$, and $z$ Components of the Pair Fe-Fe Displacements $\delta \mathrm{Fe}-\mathrm{Fe} \mathrm{e}^{a}$

\begin{tabular}{|c|c|c|c|c|c|c|c|}
\hline$\overline{l m n}$ & $\Delta X\left(\sigma_{\text {Total }}\right)(\AA)$ & $\sigma \sqrt{n}$ & $\sigma\left(f^{\prime}\right) \pm 0.2 \mathrm{eu}$ & $\sigma\left(P_{0}\right) \pm 1 \%$ & $\sigma(\mathrm{RRS}) \pm 1 \mathrm{eu}$ & $\sigma_{\text {Compton }}$ & $\sigma(C \mathrm{~A}) \pm 0.3$ at. $\%$ \\
\hline 110 & $0.0211(25)$ & 0.0002 & 0.0023 & 0.0007 & 0.0002 & 0.0004 & 0.0004 \\
\hline 200 & $-0.0228(14)$ & 0.0004 & 0.0010 & 0.0007 & 0.0002 & 0.0004 & 0.0002 \\
\hline 211 & $0.0005(2)$ & 0.0002 & 0 & 0.0001 & 0.0001 & 0 & 0 \\
\hline 121 & $0.0014(4)$ & 0.0001 & 0.0003 & 0.0001 & 0.0002 & 0 & 0 \\
\hline 220 & $0.0030(7)$ & 0.0002 & 0.0006 & 0.0001 & 0.0003 & 0.0001 & 0 \\
\hline 310 & $0.0022(3)$ & 0.0002 & 0.0001 & 0.0001 & 0.0002 & 0.0001 & 0 \\
\hline 130 & $0.0009(2)$ & 0.0002 & 0.0001 & 0 & 0.0001 & 0 & 0 \\
\hline 222 & $0.0003(3)$ & 0.0002 & 0.0002 & 0 & 0.0001 & 0 & 0 \\
\hline 321 & $0.0011(2)$ & 0.0001 & 0.0001 & 0 & 0.0002 & 0 & 0 \\
\hline 231 & $0.0001(1)$ & 0.0001 & 0 & 0 & 0.0001 & 0 & 0 \\
\hline 123 & $0.0008(4)$ & 0.0001 & 0.0001 & 0 & 0 & 0 & 0 \\
\hline 400 & $-0.0019(6)$ & 0.0004 & 0.0002 & 0.0001 & 0.0003 & 0.0001 & 0 \\
\hline 330 & $0.0011(4)$ & 0.0002 & 0.0001 & 0 & 0.0003 & 0 & 0 \\
\hline 411 & $-0.0008(3)$ & 0.0002 & 0.0002 & 0 & 0.0002 & 0 & 0 \\
\hline 141 & $-0.0001(2)$ & 0.0001 & 0.0001 & 0 & 0.0001 & 0 & 0 \\
\hline
\end{tabular}

${ }^{a}$ For the various atom pairs of $\mathrm{Fe}_{46.5} \mathrm{Ni}_{53.5}$ for statistical and possible systematic errors described in the text. Total error is shown in parentheses and 0 indicates uncertainties $<0.00005 \AA$. 


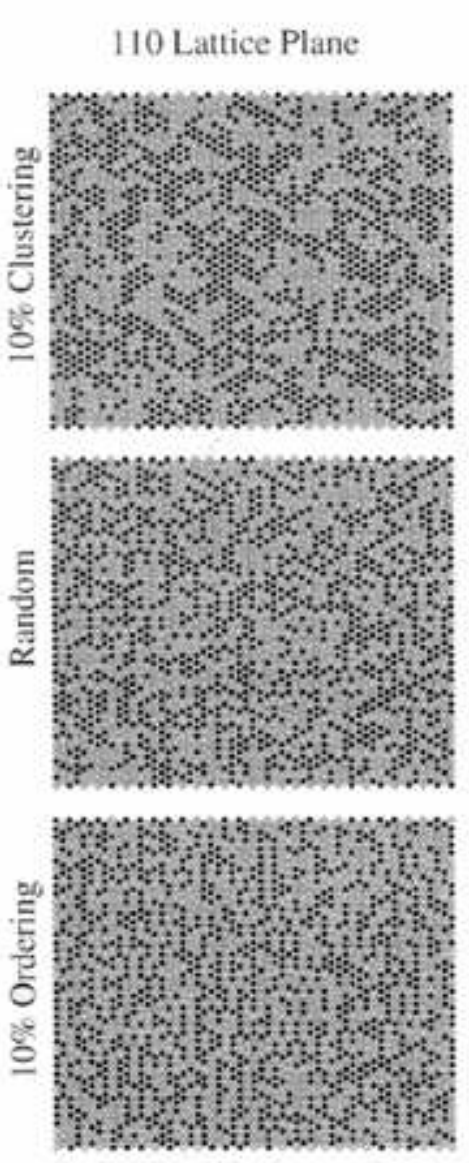

100 Diffraction Plane
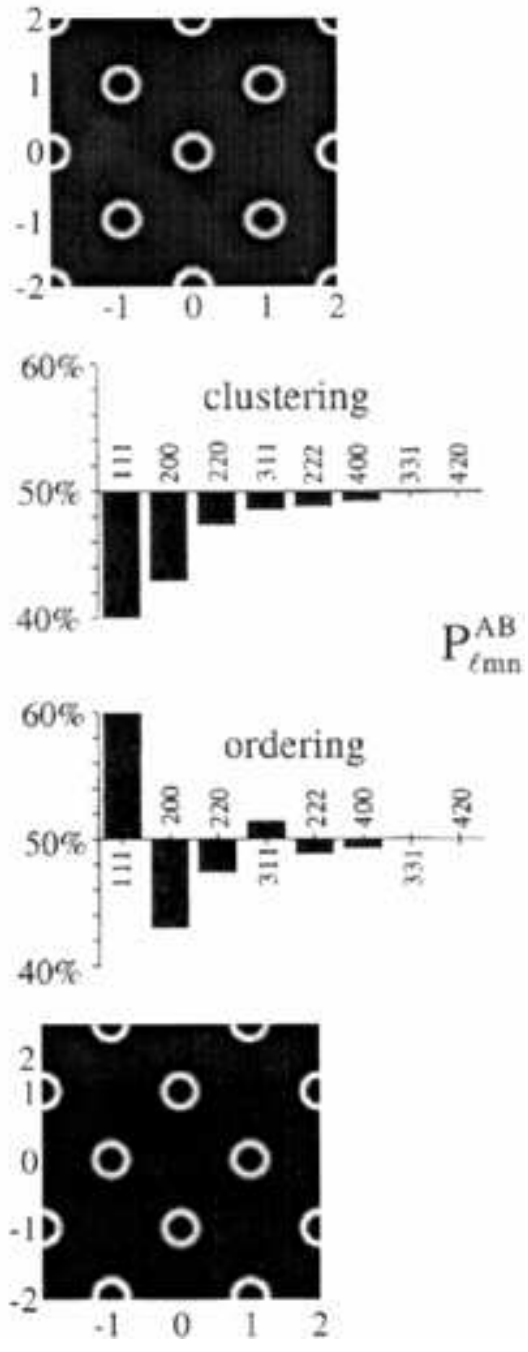

$146 \times 188 \mathrm{~mm}(72 \times 72$ DPI $)$ 


1
2
3
4
5
6
7
8
9
10
11
12
13
14
15
16
17
18
19
20
21
22
23
24
25
26
27
28
29
30
31
32
33
34
35
36
37
38
39
40
41
42
43
40
45
49
50
50
51
53
55
50

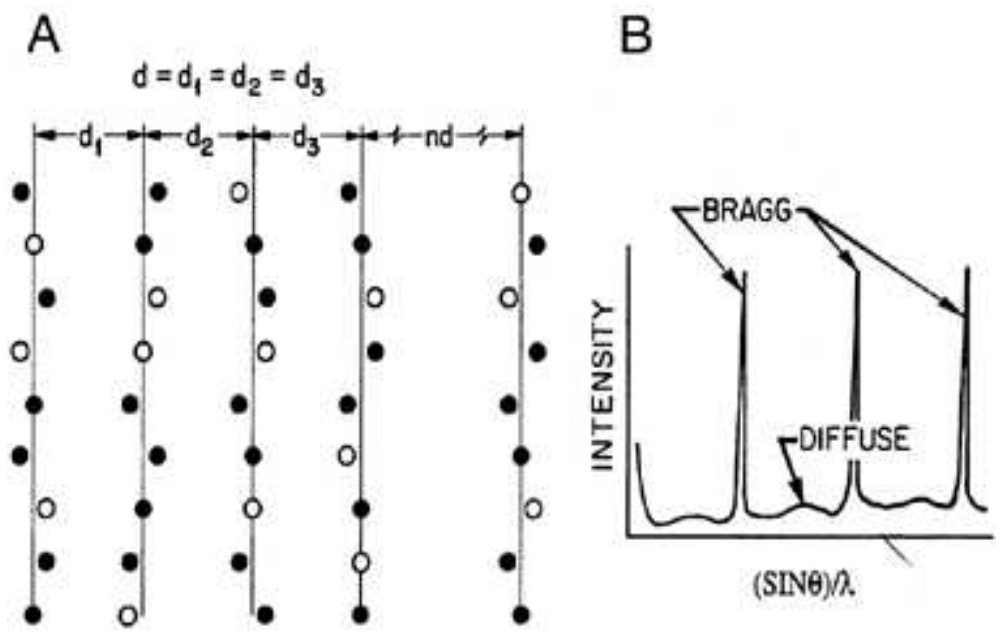

$133 \times 85 \mathrm{~mm}(72 \times 72$ DPI $)$ 


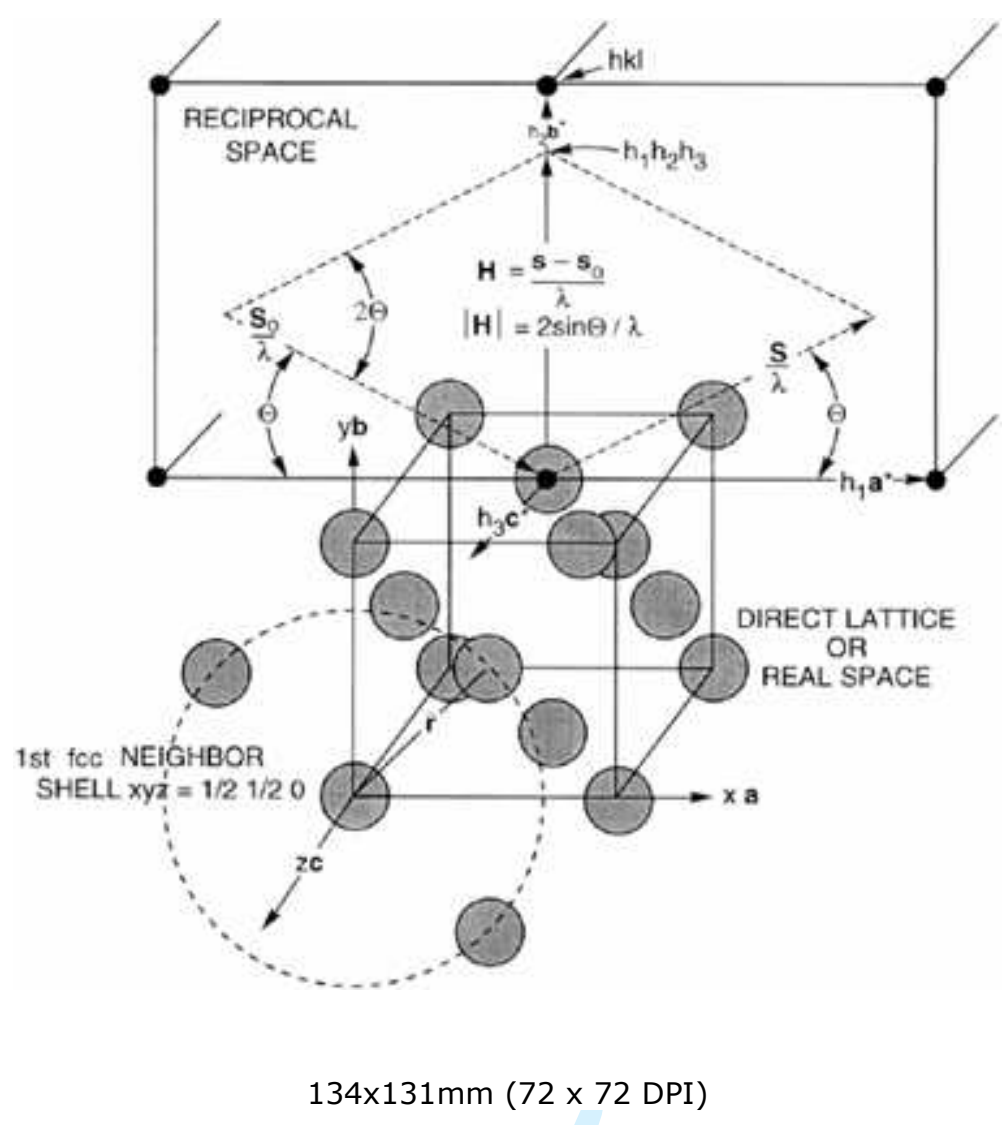




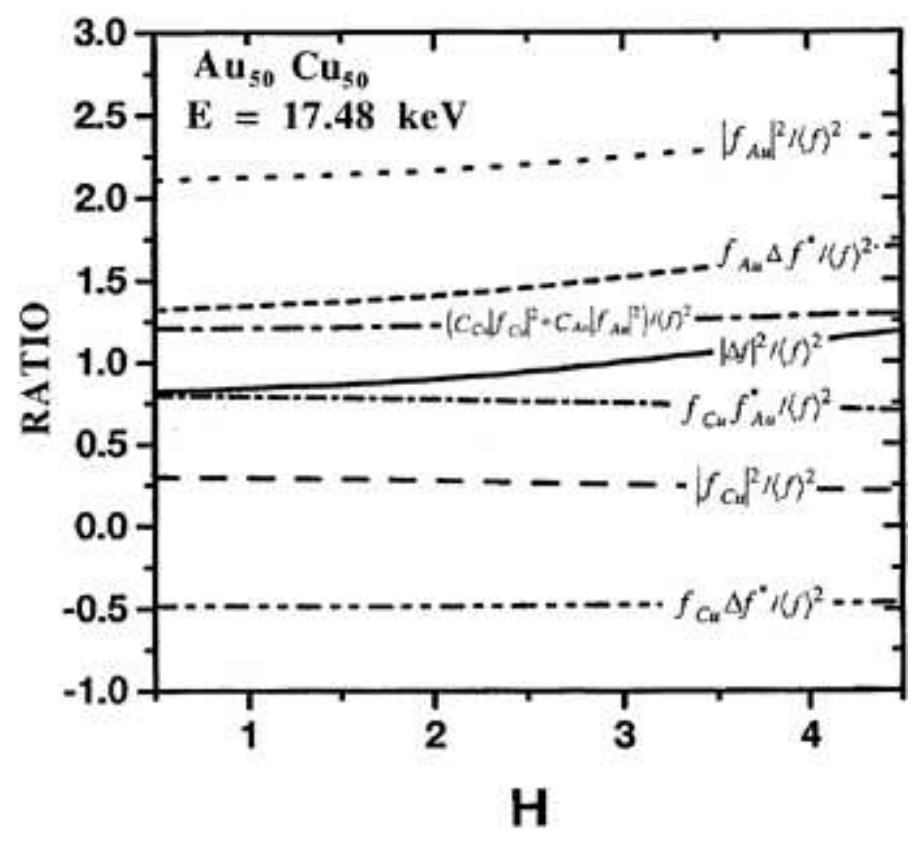

$120 \times 110 \mathrm{~mm}(72 \times 72$ DPI $)$ 


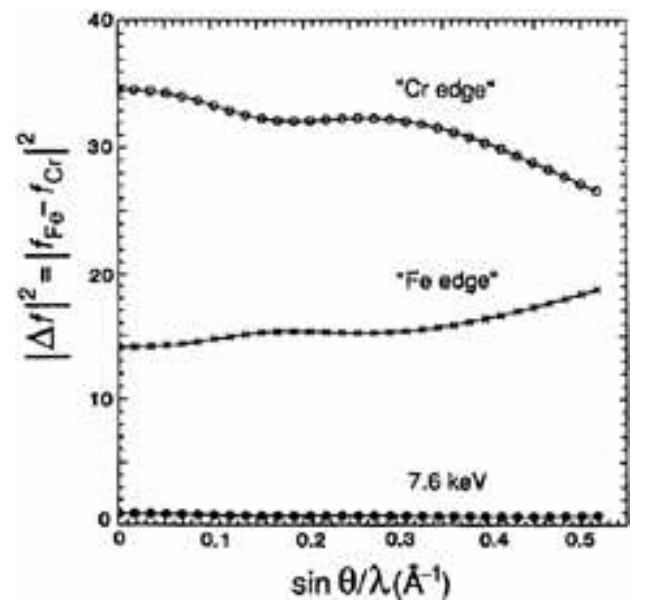

$83 \times 80 \mathrm{~mm}(72 \times 72$ DPI $)$

John Wiley \& Sons 


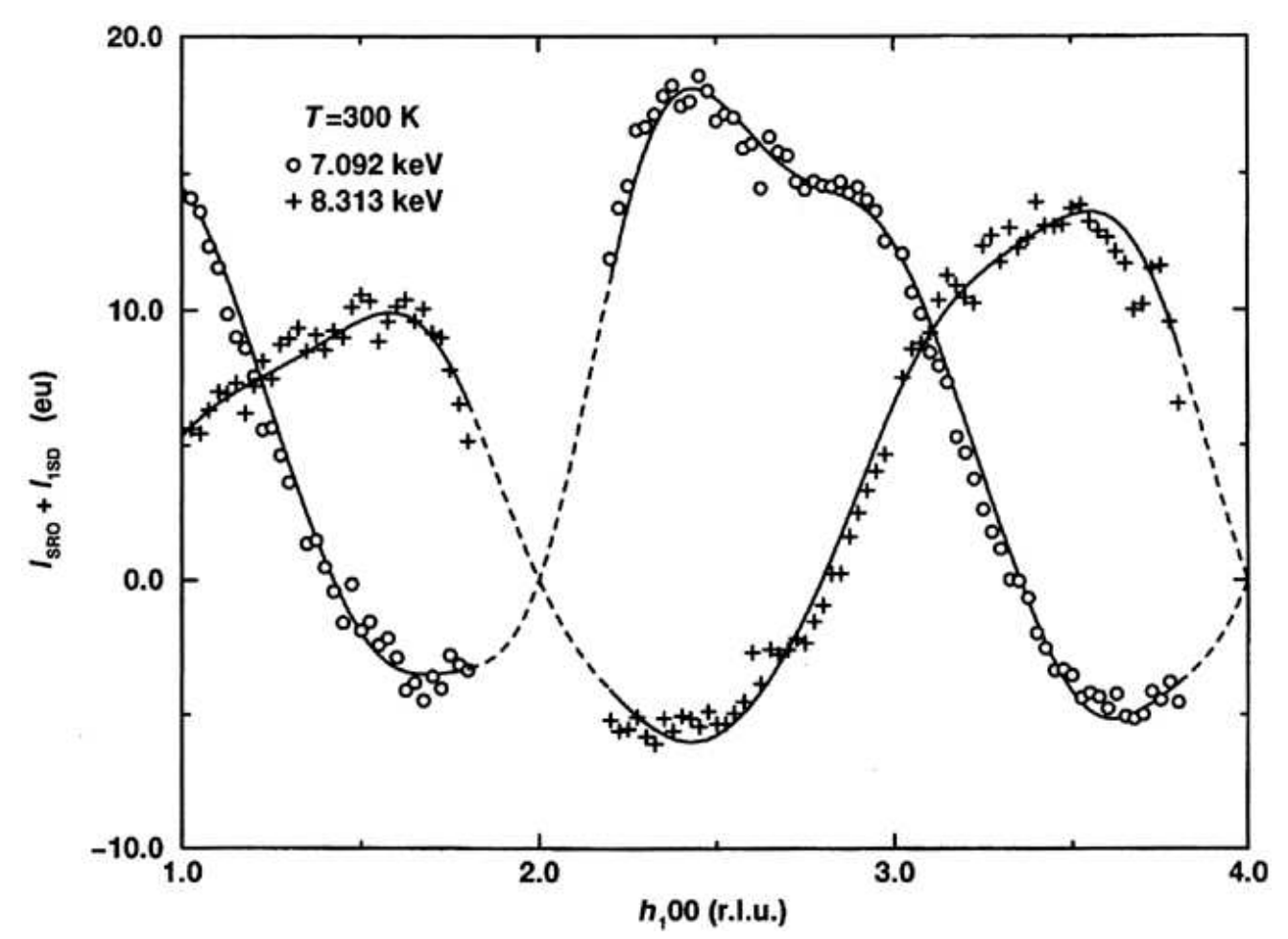

$234 \times 170 \mathrm{~mm}(72 \times 72 \mathrm{DPI})$ 


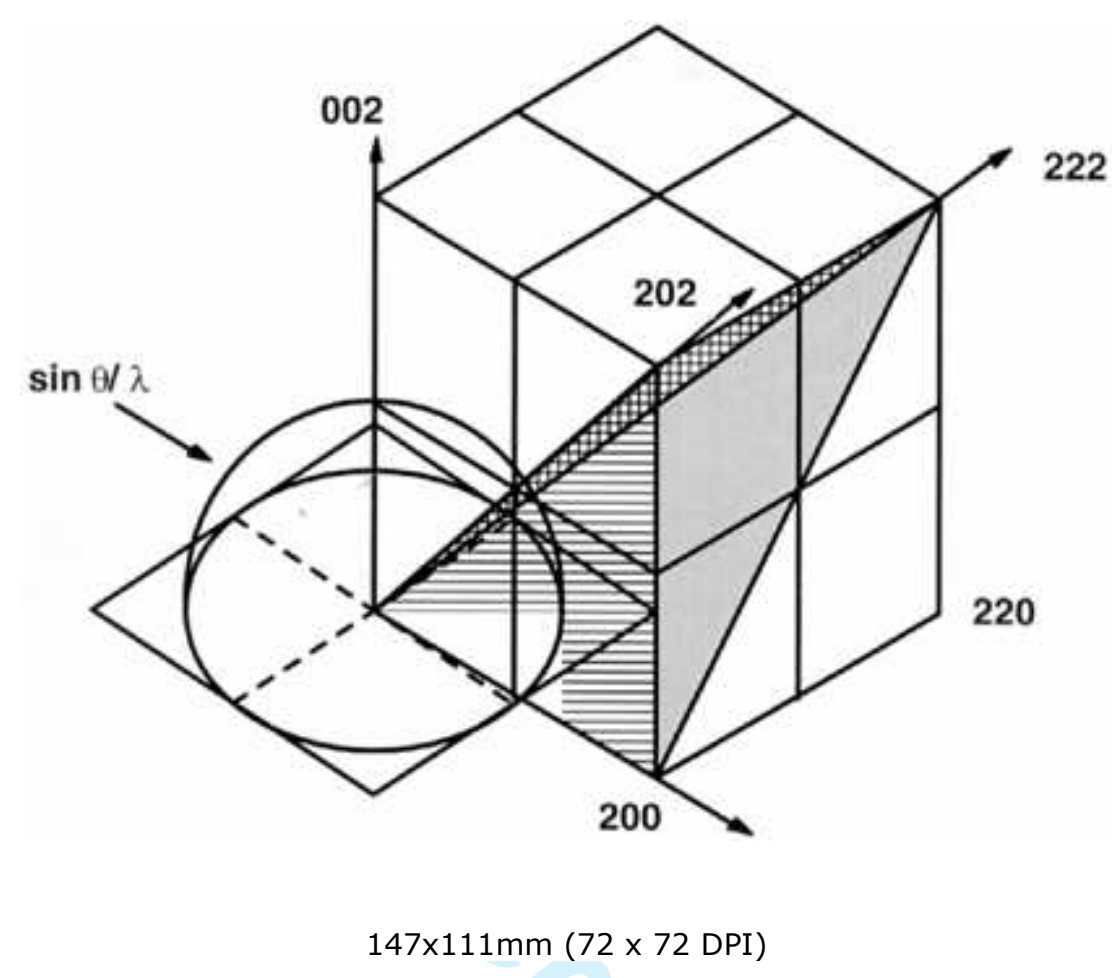

John Wiley \& Sons 


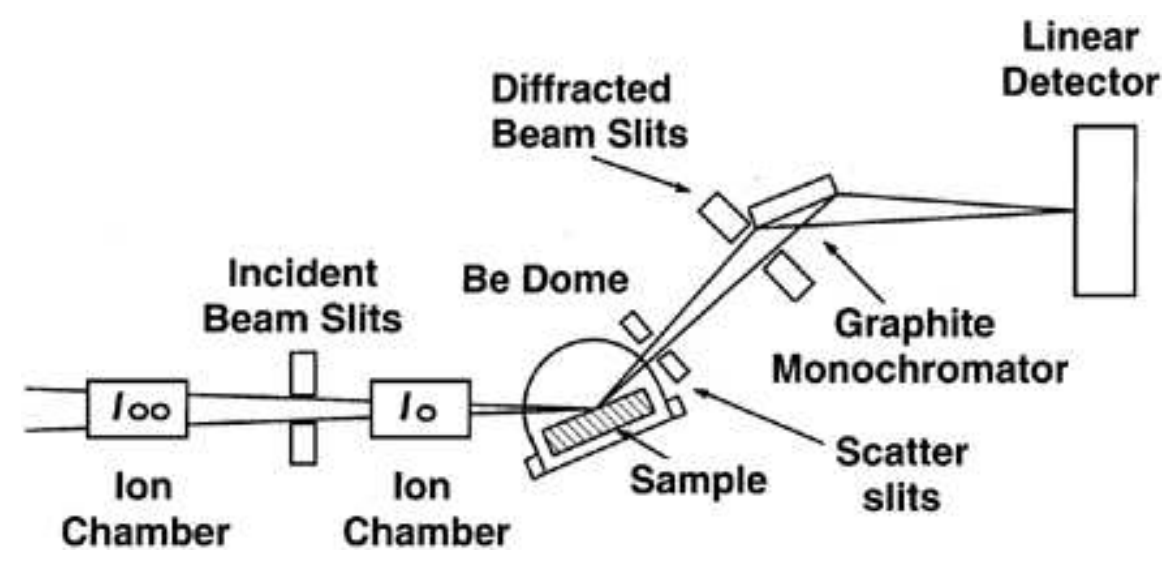

$154 \times 72 \mathrm{~mm}(72 \times 72 \mathrm{DPI})$ 


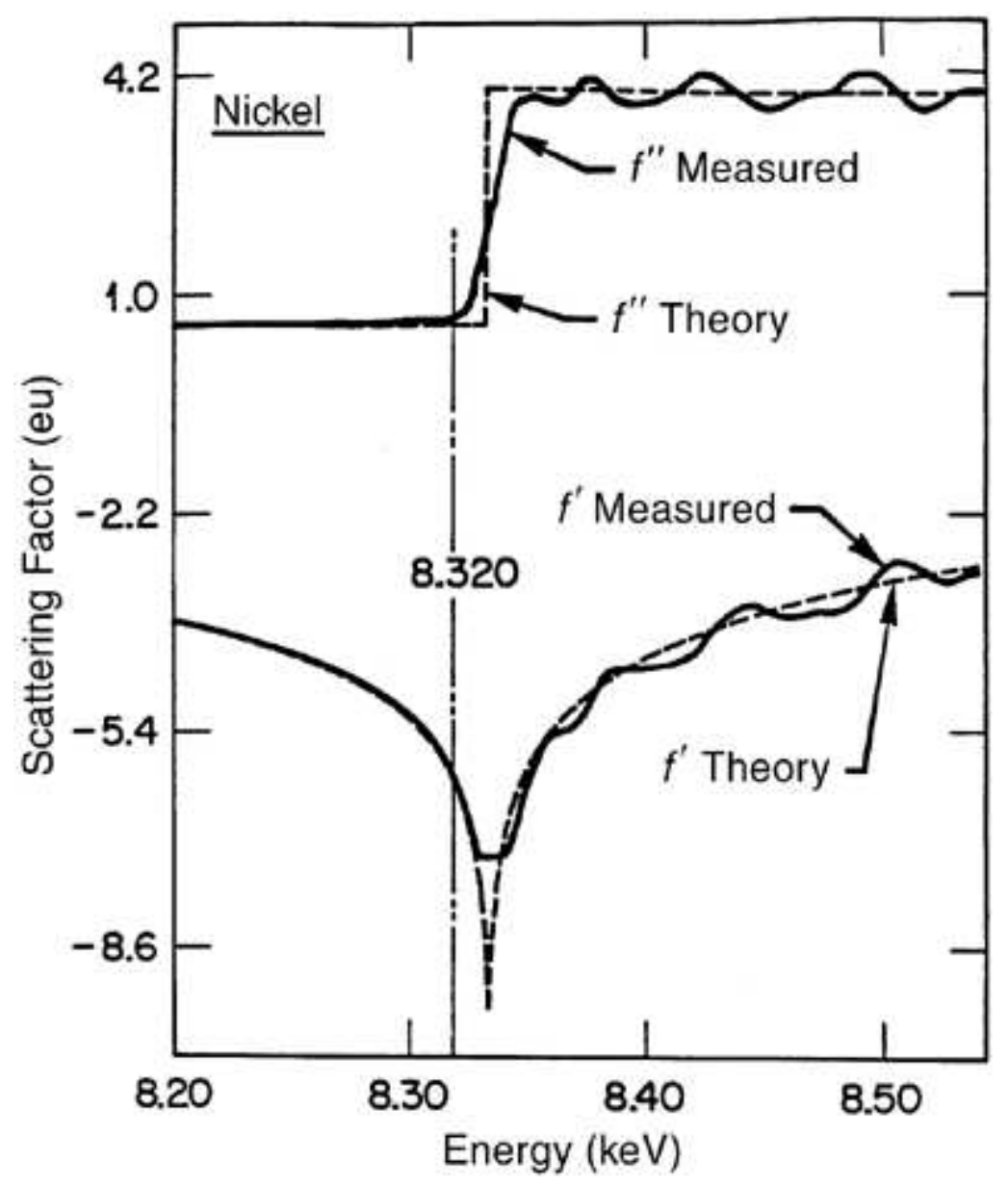

$137 \times 164 \mathrm{~mm}(72 \times 72$ DPI $)$ 


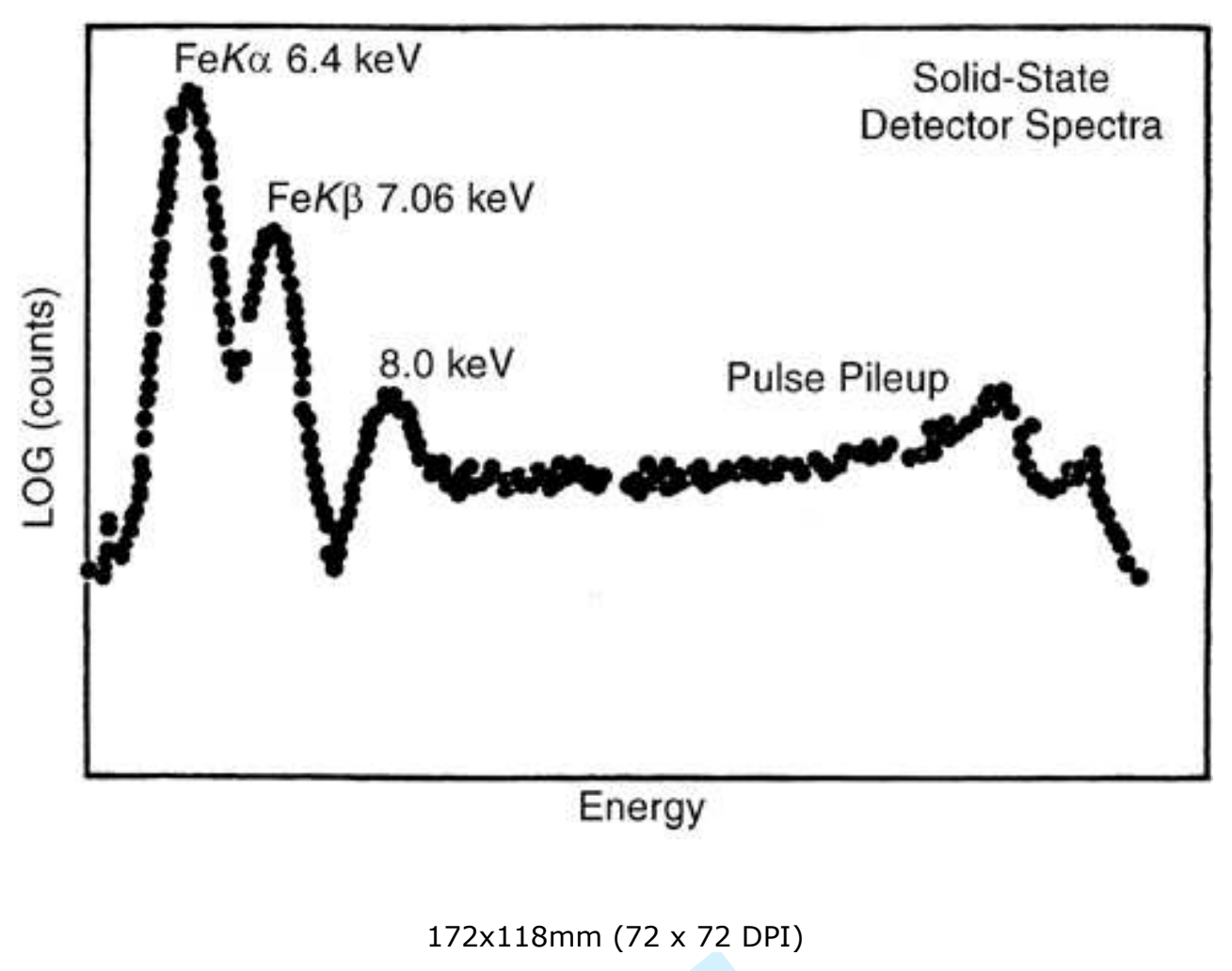

John Wiley \& Sons 


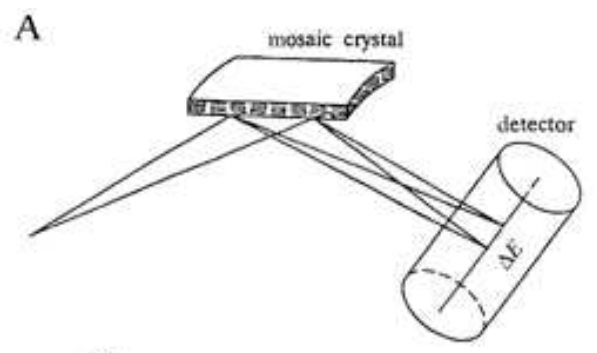

B

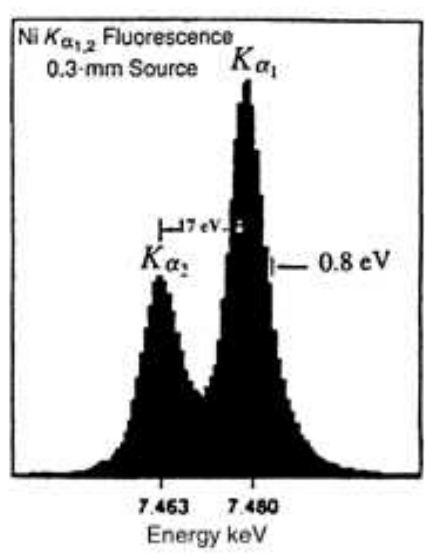

C

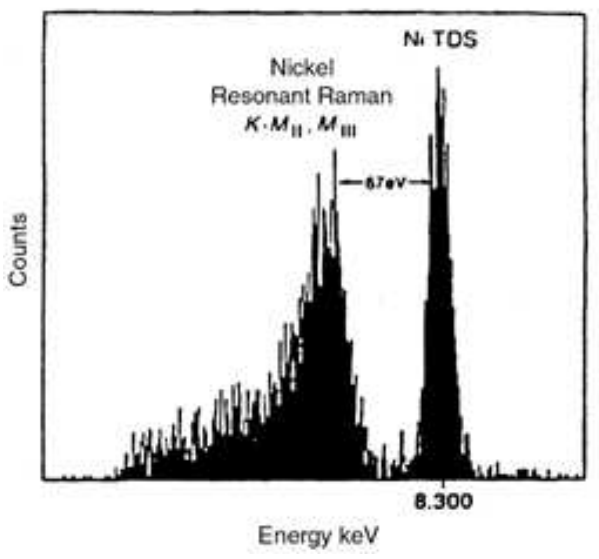

$92 \times 234 \mathrm{~mm}(72 \times 72$ DPI $)$ 


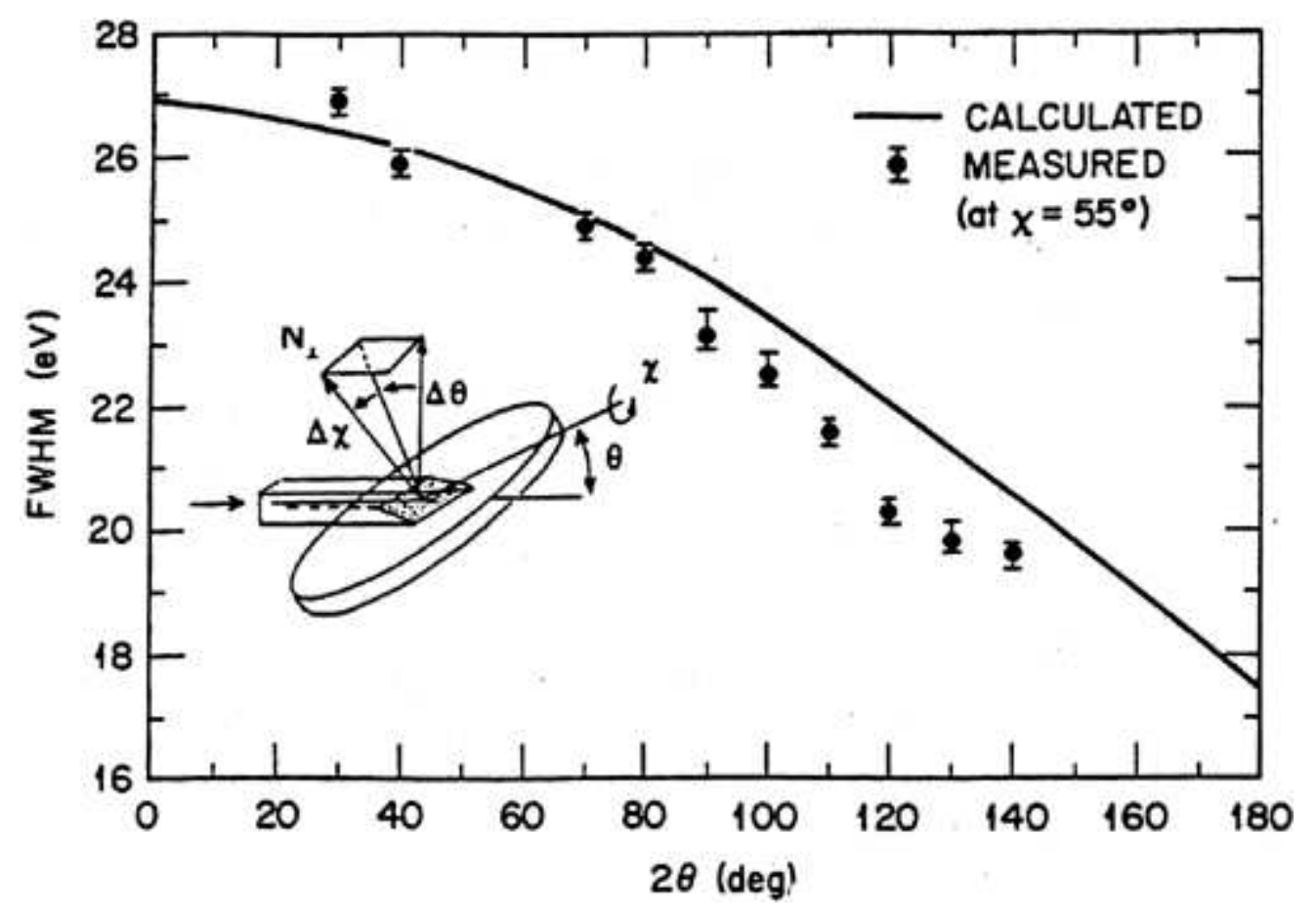

$176 \times 122 \mathrm{~mm}(72 \times 72$ DPI $)$ 

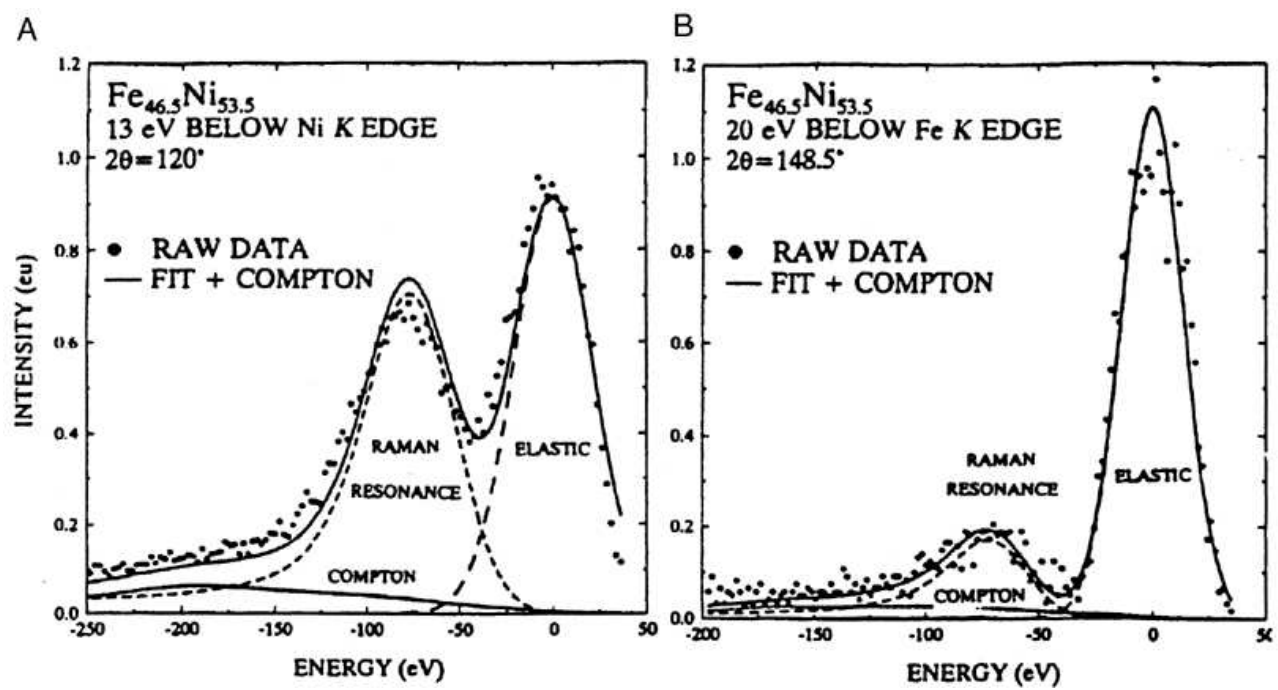

$291 \times 152 \mathrm{~mm}(72 \times 72$ DPI $)$ 


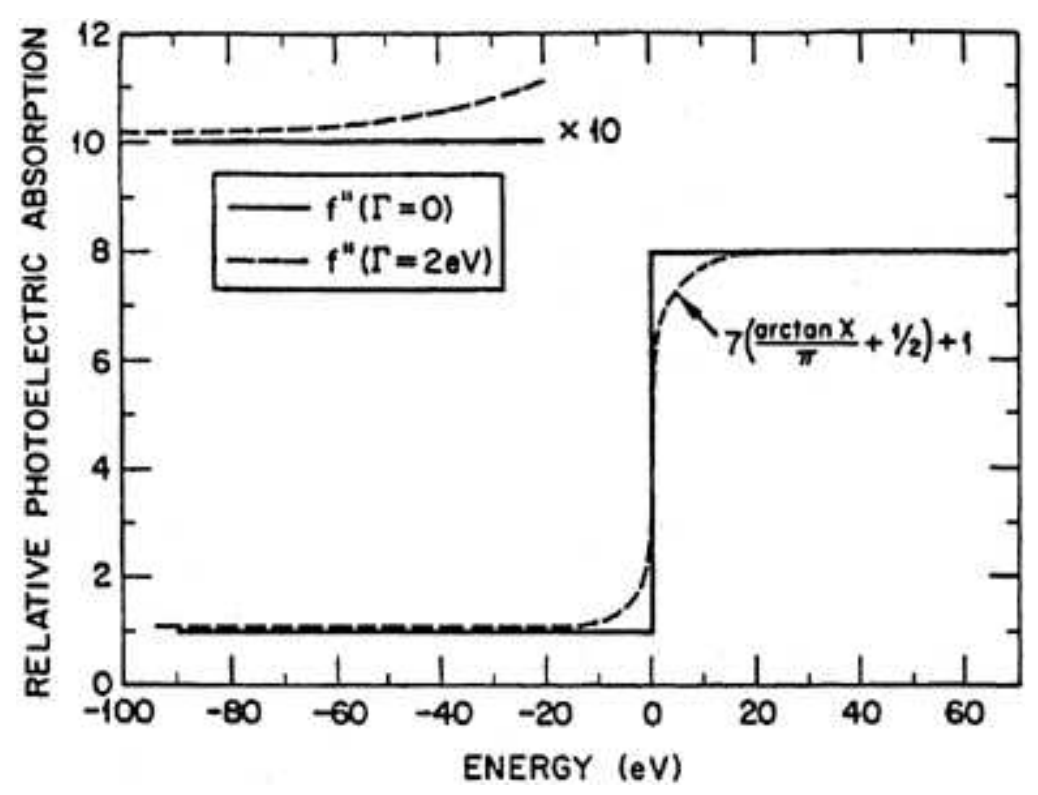

$136 \times 103 \mathrm{~mm}(72 \times 72$ DPI $)$ 


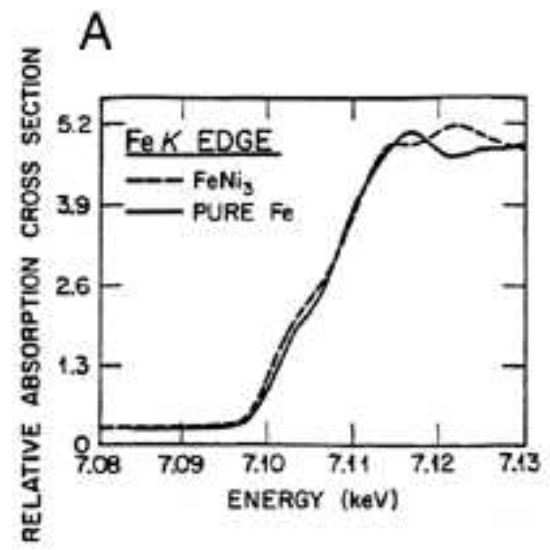

B

1

2

3

4

5

6
7

8

9

10

11

12

13

14

15

16

17

18

19

20

21

22

23

24

25

26

27

28

29

30

31

32

33

34

35

36

37

38

39

40

41

42

43

44

45

46

47

48

49

50

51

52

53

54

55

56

57

58

59

60

$145 \times 73 \mathrm{~mm}(72 \times 72 \mathrm{DPI})$

John Wiley \& Sons 


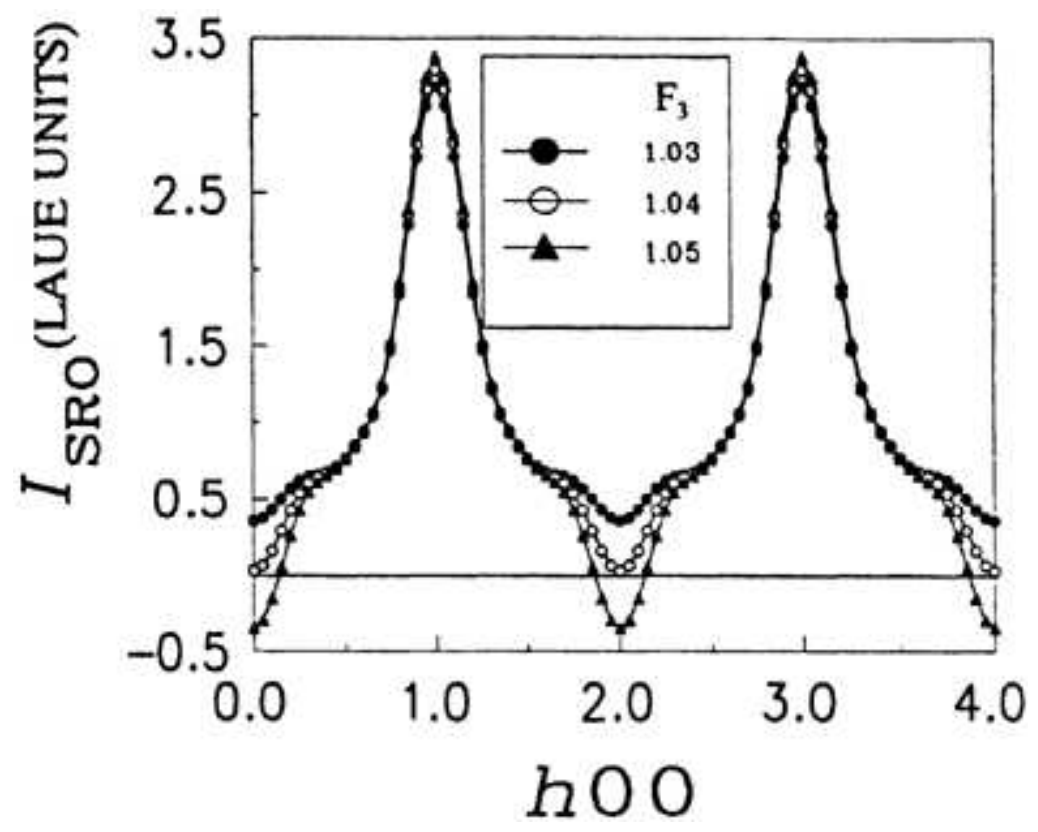

$137 \times 110 \mathrm{~mm}(72 \times 72$ DPI $)$ 


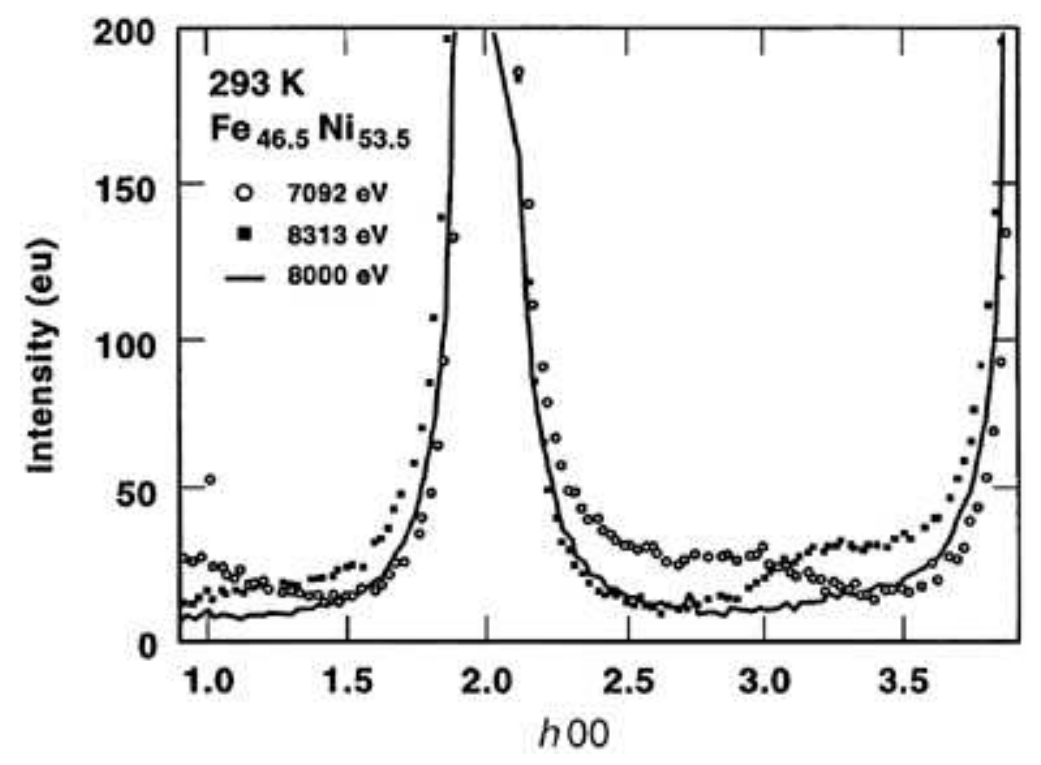

$136 \times 100 \mathrm{~mm}(72 \times 72$ DPI $)$ 

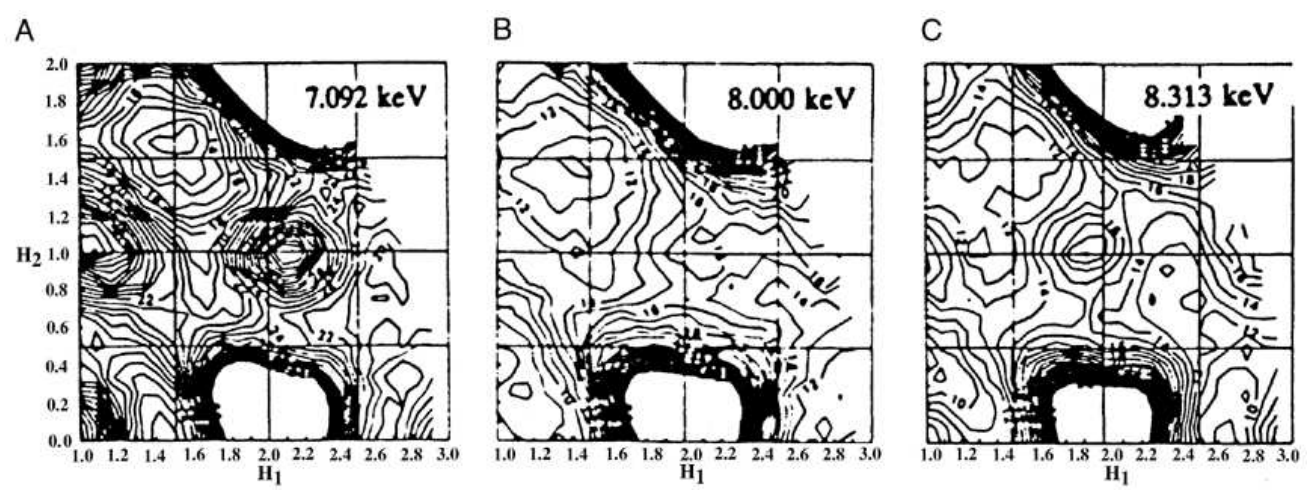

$304 \times 112 \mathrm{~mm}(72 \times 72$ DPI $)$ 


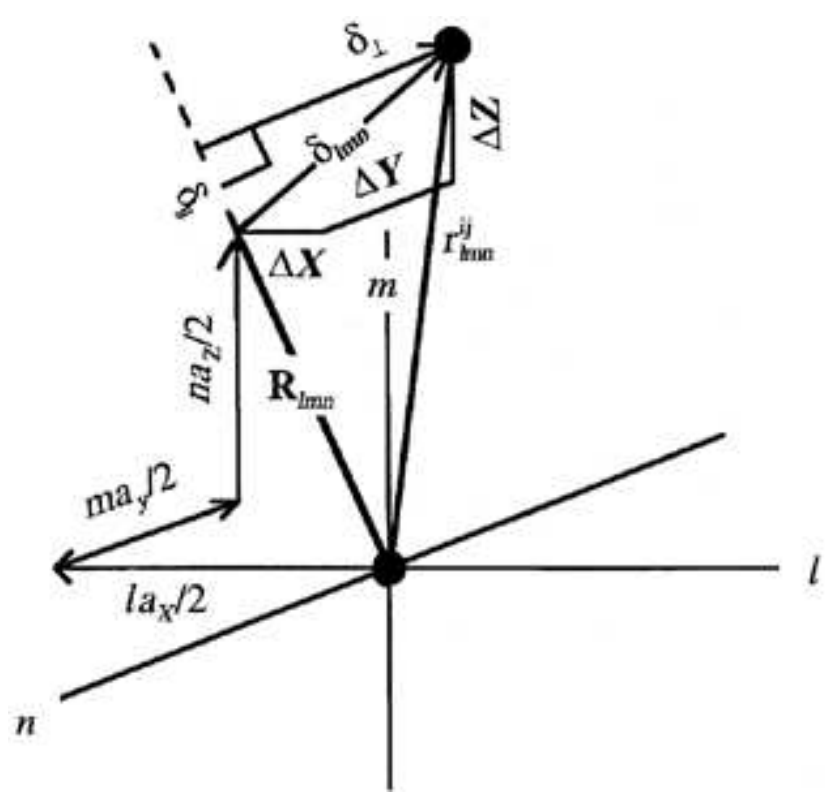

110x105mm (72 x 72 DPI) 


1
2
3
4
5
6
7
8
9
10
11
12
13
14
15
16
17
18
19
20
21
22
23
24
25
26
27
28
29
30
31
32
33
34
35
36
37
38
39
40
41
42
43
40
45
49
50
50
51
53
55
50

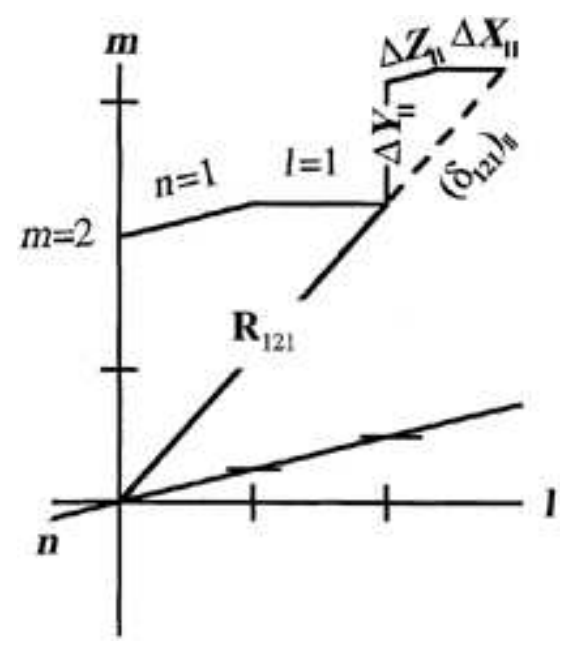

$73 \times 84 \mathrm{~mm}(72 \times 72 \mathrm{DPI})$ 

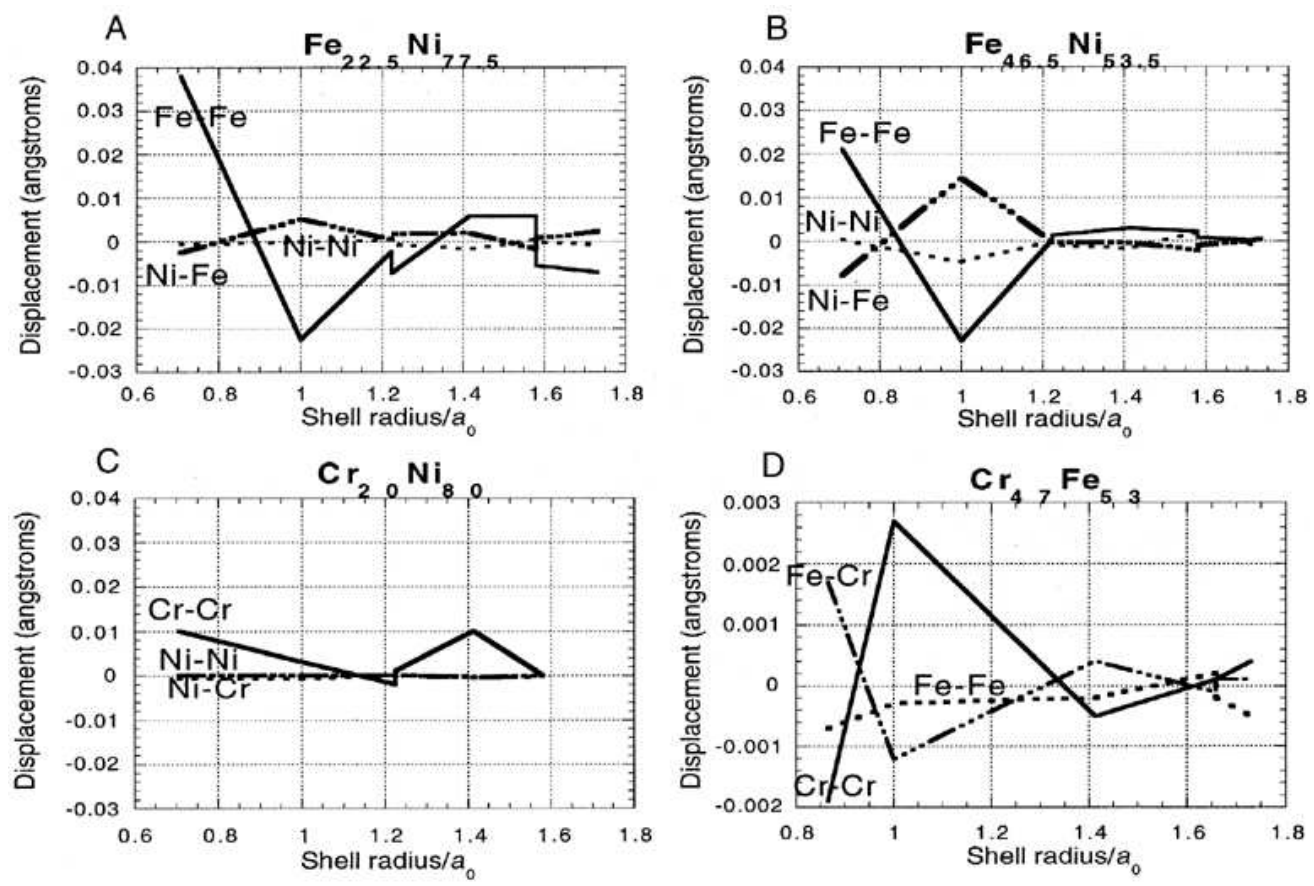

$271 \times 183 \mathrm{~mm}(72 \times 72$ DPI $)$ 


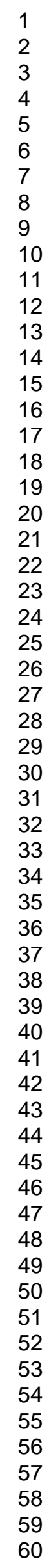

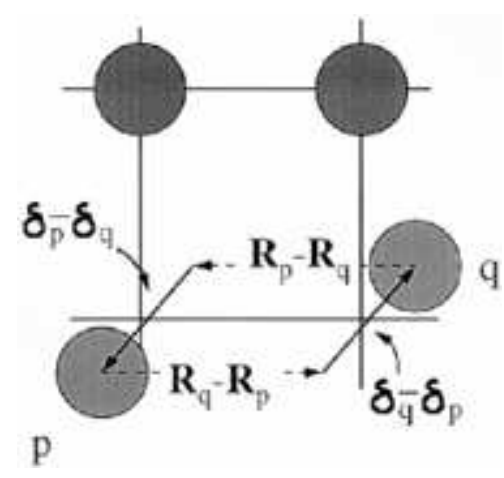

$65 \times 61 \mathrm{~mm}(72 \times 72 \mathrm{DPI})$ 


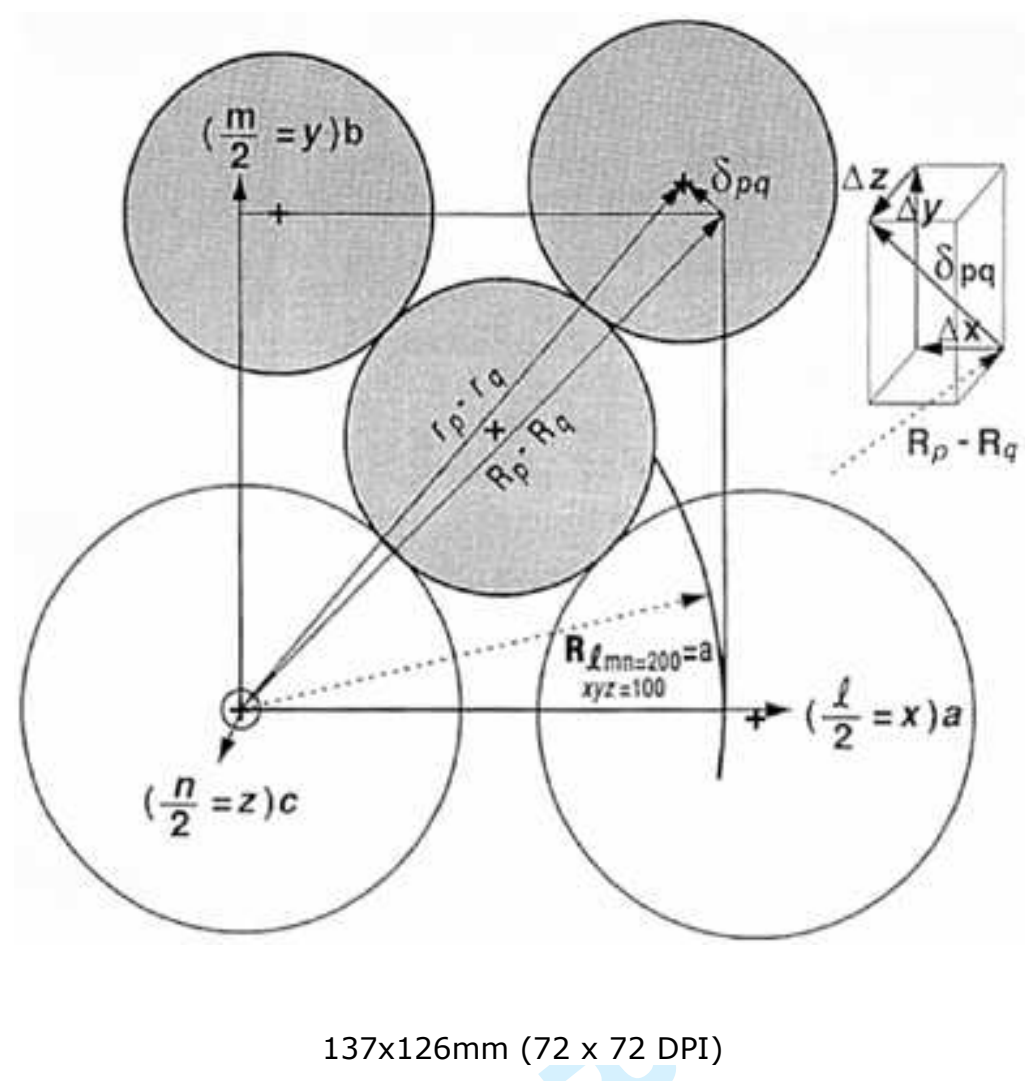

33

34

35

36

37

38

39

40

41

42

43

44

45

46

47

48

49

50

51

52

53

54

55

56

57

58

59

60

John Wiley \& Sons 